LBNL-44155

\title{
Semianalytical Solutions of RADIOACTIVE OR REACTIVE TRACER TRANSPORT IN LAYERED Fractured Media
}

\section{G. J. Moridis and G. S. Bodvarsson}

Earth Sciences Division

Lawrence Berkeley National Laboratory

Berkeley, CA 94720

\section{October 2001}

This work was supported by the Director, Office of Civilian Radioactive Waste Management, U.S. Department of Energy, through Memorandum Purchase Order EA9013MC5X between Bechtel SAIC Company, LLC and the Ernest Orlando Lawrence Berkeley National Laboratory (Berkeley Lab). The support is provided to Berkeley Lab through the U.S. Department of Energy Contract No. DE-AC03-76SF00098. 



\title{
Semianalytical Solutions of Radioactive or Reactive Tracer Transport in Layered Fractured Media
}

\begin{abstract}
In this paper, semianalytical solutions are developed for the problem of transport of radioactive or reactive tracers (solutes or colloids) through a layered system of heterogeneous fractured media with misaligned fractures. The tracer transport equations in the matrix account for (a) diffusion, (b) surface diffusion (for solutes only), (c) mass transfer between the mobile and immobile water fractions, (d) linear kinetic or equilibrium physical, chemical, or combined solute sorption or colloid filtration, and (e) radioactive decay or first order chemical reactions. Any number of radioactive decay daughter products (or products of a linear, first-order reaction chain) can be tracked. The tracer-transport equations in the fractures account for the same processes, in addition to advection and hydrodynamic dispersion. Additionally, the colloid transport equations account for straining and velocity adjustments related to the colloidal size. The solutions, which are analytical in the Laplace space, are numerically inverted to provide the solution in time and can accommodate any number of fractured and/or porous layers. The solutions are verified using analytical solutions for limiting cases of solute and colloid transport through fractured and porous media. The effect of important parameters on the transport of ${ }^{3} \mathrm{H},{ }^{237} \mathrm{~Np}$ and ${ }^{239} \mathrm{Pu}$ (and its daughters) is investigated in several test problems involving layered geological systems of varying complexity. ${ }^{239} \mathrm{Pu}$ colloid transport problems in multilayered systems indicate significant colloid accumulations at straining interfaces but much faster transport of the colloid than the corresponding strongly sorbing solute species.
\end{abstract}




\section{Introduction}

The study of radioactive and/or reactive contaminant transport (of solutes and colloids) in complex fractured geologic systems has become increasingly important in recent years because of the need to predict the migration and fate of the contaminants. Currently, there are some very large contaminated sites (such as Hanford, Washington; Nevada Test Site (NTS), Nevada; Idaho National Engineering and Environmental Laboratory (INEEL), Idaho) where severe pollution by radioactive materials extends over large areas within the subsurface rocks. Experience with numerical model predictions using reactive chemical codes has indicated that much research is needed in this area, because field observations have shown that contaminated plumes can move much faster than models have predicted [Mc Carthy and Zachara, 1989; Buddemeier and Hunt, 1988, Kersting et al., 1999].

At Yucca Mountain (YM), Nevada, the site of the potential repository for high-level nuclear waste, the potential transport of radioactive contaminants must be predicted for tens to hundreds of thousands of years. Performing reliable radionuclide transport calculations for this temporal and spatial scale is obviously very difficult, and furthermoreit is impossible to verify the results. In addition, the complex geology of the site and the unsaturated nature of a significant portion of the flow path add to the difficulty in making such predictions.

The potential site is located in southern Nevada about $120 \mathrm{~km}$ northwest of Las Vegas, and is characterized by a thick unsaturated zone $(600-700 \mathrm{~m})$ and the presence of rocks onto which important radionuclides in the wastes tend to sorb strongly. The YM stratigraphy consists of layers of welded and nonwelded tuffs (with vastly different hydraulic, transport, and geochemical properties), with the former generally being extensively fractured and the latter behaving similarly to a porous medium [Montazer and Wilson, 1984; Liu et al., 1998; Bandurraga and Bodvarsson, 1999].

The extremely varied geological and hydrological characteristics of the different tuff 
layers at Yucca Mountain make the modeling of flow and transport a formidable task. A single representation for all of the hydrogeologic units is inappropriate, and several different approaches and algorithms must be employed for reliable modeling results. Analytical and semianalytical models of transport that can account for the site heterogeneity are important because they allow the validation of complex multidimensional numerical models, are computationally efficient, and can provide bounding estimates of the possible solutions of the expected transport at the site.

Previous analytical solutions of solute and colloid transport infractured media involved exclusively single semi-infinite domains (layers). Tang et al. [1981] developed a quasi twodimensional solution for the transport of solutes in a single saturated fracture (i.e., with a semi-infinite matrix) that assumed a constant concentration boundary and accounted for (a) advection and dispersionin thefractures, (b) diffusion inthe matrix, thefractures, and across their interface, (c) sorption onto the matrix and the fractures, and (d) radioactive decay. The analytical solution of Sudicky and Frind [1982] accounted for the same processes in solute transport in a system of parallel fractures (i.e., with a finite matrix block size). The solution of Robinson et al. [1998] is an extension of the Sudicky and Frind [1982] solution and accounts for the effect of fracture skin on transport in a system of parallel fractures. By neglecting hydrodynamic dispersion in the fractures and assuming an instantaneous (delta Dirac-type) deposition of a parent radionuclide at the boundary, Sudicky and Frind [1984] obtained analytical solutions to the problem of transport of a two-member radioactive chain in a single fracture.

Abdel-Salam and Chrysikopoulos [1994] developed a set of analytical solutions to the problem of nonradioactive colloid transport in a single saturated fracture for different boundary conditions. These solutions account for (a) advection and dispersion in the fractures, (b) diffusion in the matrix, the fractures, and across their interface, and (c) kinetic irreversible filtration in the fractures and the matrix. 
In this paper, semianalytical solutions are developed for the problem of transport of radioactiveorreactivetracers(solutesorcolloids)throughalayeredsystemofheterogeneous fractured media with misaligned fractures (such as the unsaturated zone at YM). The solutions allow any number and combination of fractured and/or porous layers that can vary in hydraulic and transport properties, fracture frequency, water saturation, fracture flow, and fracture-matrix interaction. The tracer transport equations in the matrix account for (a) diffusion (molecular or colloidal), (b) surface diffusion (for solutes only), (c) mass transfer between the mobile and immobile water fractions, (d) linear kinetic or equilibrium physical,chemical orcombinedsolutesorptionorcolloidfiltration, and (e)radioactivedecay or first order chemical reactions. Any number of daughter products of radioactive decay (or of a linear, first-order reaction chain) can be tracked. The tracer transport equations in the fractures account for the same processes, in addition to advection and hydrodynamic dispersion. Additionally, the colloid transport equations account for straining and velocity adjustments related to the colloidal size. The solutions, which are analytical in the Laplace space, are numerically inverted to provide the solution in time, and can accommodate constant or time-variable concentration or flux boundary conditions.

\section{Solute Transport Equations}

\subsection{The PDE of Solute Transport}

The1-DPDE of transport of aradioactiveor reactivesolutethrough avariablysaturated porous or fractured medium (PM or FM) is described by the equation

$$
\begin{aligned}
& D_{m} \frac{\partial^{2} C}{\partial x^{2}}+D_{i} \frac{\partial^{2} C_{i}}{\partial x^{2}}+D_{F} \frac{\partial^{2} F}{\partial x^{2}}-U \frac{\partial C}{\partial x} \\
& =\phi\left(S-S_{r}\right)\left(\frac{\partial C}{\partial t}+\delta_{r} \frac{\partial \mathcal{R}}{\partial t}\right)+\phi S_{r}\left(\frac{\partial C_{i}}{\partial t}+\delta_{r} \frac{\partial \mathcal{R}_{i}}{\partial t}\right)+(1-\phi) \rho \frac{\partial F}{\partial t} \\
& \quad+\lambda \delta_{\lambda}\left[\phi\left(S-S_{r}\right) C+\phi S_{r} C_{i}+(1-\phi) \rho F\right],
\end{aligned}
$$

where 
C dissolved species concentration in the mobile pore water $\left[M L^{-3}\right]$;

$D_{m} \quad$ intrinsic diffusion coefficient for the mobile pore water $\left[L^{2} T^{-1}\right]$;

$C_{i} \quad$ dissolved species concentration in the immobile pore water $\left[M L^{-3}\right]$;

$D_{i} \quad$ intrinsic diffusion coefficient in the immobile pore water $\left[L^{2} T^{-1}\right]$;

$F \quad=F_{p}+F_{c}$

$F_{p}$ relative concentration of the physically adsorbed species $\left[\left(M L^{-3}\right) /\left(M L^{-3}\right)\right]$;

$F_{c} \quad$ relative concentration of the chemically sorbed species $\left[\left(M L^{-3}\right) /\left(M L^{-3}\right)\right]$;

$\mathcal{R}$ reacted species mass per unit volume in the mobile fraction $\left[M L^{-3}\right]$;

$\mathcal{R}_{i} \quad$ reacted species mass per unit volume in the immobile fraction $\left[M L^{-3}\right]$;

$D_{F} \quad$ apparent surface diffusion coefficient $\left[M L^{-1} T^{-1}\right]$;

$U=f_{v} V \phi\left(S-S_{r}\right)$, Darcy velocity $\left[L T^{-1}\right] ;$

$V \quad$ pore flow velocity $\left[L T^{-1}\right]$;

$f_{v} \quad$ velocity adjustment factor ( $=1$ for solutes, see discussion in Section 3.1);

$S \quad$ water saturation $\left[L^{3} / L^{3}\right]$;

$S_{r} \quad$ irreducible water saturation $\left[L^{3} / L^{3}\right]$;

$\rho \quad$ PM grain density $\left[M L^{-3}\right]$;

$\phi \quad$ total PM porosity $\left[L^{3} / L^{3}\right]$;

$\lambda \quad=\ln 2 / T_{1 / 2}$, radioactive decay constant $\left[T^{-1}\right] ;$

$T_{1 / 2} \quad$ half-life of radioactive species [T].

The parameters $\delta_{s}$ and $\delta_{\lambda}$ are defined as

$$
\delta_{r}=\left\{\begin{array}{ll}
1 & \text { for reactive transport } \\
0 & \text { for radionuclide transport }
\end{array} \text { and } \delta_{\lambda}= \begin{cases}0 & \text { for reactive transport } \\
1 & \text { for radionuclide transport }\end{cases}\right.
$$

The first three terms on the left-hand side of (1) describe diffusion in the mobile pore water [Skagius and Neretnieks, 1988], through the immobile thin film in the immediate vicinity of the PM grains [de Marsily, 1986], and surface diffusion [Jahnke and Radke, 1987; Skagius and Neretnieks, 1988; Cook, 1989; Berry and Bond, 1992], respectively. 
The fourth term on the left-hand side (1) describes advective transport. The terms on the right-hand side of equation (1) describe the dissolved species accumulation and radioactive decay in the pore water, in the immobile fraction, and on the PM grains due to sorption (for solutes) or filtration (for colloids). Chemical reactions in the water phase are also accounted for [Cho, 1971]. A detailed discussion of these terms can be found in Moridis [1999], from where

$$
D_{m}=\phi\left(S-S_{r}\right)\left(\tau_{p} D_{0}+\alpha_{L} f_{v} V\right) \quad \text { and } \quad D_{i}=\tau_{i} \phi S_{r} D_{0}
$$

where $D_{0}$ is the molecular diffusion coefficient of the dissolved species in water $\left[L^{2} T^{-1}\right]$, $\alpha_{L}$ is the longitudinal dispersivity $[L], \tau_{p}$ is the tortuosity factor of the pore paths [dimensionless], and $\tau_{i}$ is the tortuosity factor in the diffusion paths through the immobile fraction [dimensionless]. If surface diffusion cannot beneglected [Jensen and Radke, 1988], $D_{F}$ is given by [Jahnke, 1986; Jahnke and Radke, 1987]

$$
D_{F}=\tau_{s}(1-\phi) \rho D_{s}
$$

where $\tau_{s}$ is the tortuosity coefficient of the surface path [dimensionless], and $D_{s}$ is the surface diffusion coefficient $\left[L^{2} T^{-1}\right]$. For homogeneous PM systems there is theoretical justification [Cook, 1989] for the relationship $\tau_{s}=\frac{2}{3} \tau_{p}$.

The species concentration in the mobile and immobile water fractions are related through the linear equilibrium relationship [de Marsily, 1986],

$$
C_{i}=K_{i} C, \quad \mathcal{R}_{i}=K_{i} \mathcal{R},
$$

where $K_{i}$ is a dimensionless mass transfer coefficient. Equation (1) then becomes

$$
\begin{aligned}
D_{T} \frac{\partial^{2} C}{\partial x^{2}} & +D_{F} \frac{\partial^{2} F}{\partial x^{2}}-U \frac{\partial C}{\partial x} \\
& =\phi h\left(\frac{\partial C}{\partial t}+\delta_{\lambda} \lambda C\right)+(1-\phi) \rho\left(\frac{\partial F}{\partial t}+\delta_{\lambda} \lambda F\right)+\delta_{r} \phi h \frac{\partial \mathcal{R}}{\partial t}
\end{aligned}
$$


where

$$
D_{T}=\phi\left\{D_{0}\left[\tau_{p}\left(S-S_{r}\right)+\tau_{i} S_{r} K_{i}\right]+\left(S-S_{r}\right) \alpha_{L} f_{v} V\right\}
$$

and

$$
h=\left(S-S_{r}\right)+S_{r} K_{i} .
$$

\subsection{The Equations of Solute Sorption and First-Order Chemical Reaction}

Consideringthat sorption occurs as thedissolvedspecies diffusesthrough the immobile water fraction, and assuming linear equilibrium (LE) sorption, the following relationship applies:

$$
F_{p}=K_{d} K_{i} C
$$

where $K_{d}$ is the distribution coefficient $\left[M^{-1} L^{3}\right]$.

Linear kinetic physical (LKP) and linear irreversible physical (LIP) sorption are described by the equation [Moridis, 1999]

$$
\frac{\partial F_{p}}{\partial t}+\lambda F_{p}=k_{p}\left(K_{d} K_{i} C-\delta_{p} F_{p}\right)
$$

where $k_{p}$ is the kinetic constant of linear adsorption $\left[T^{-1}\right]$, and

$$
\delta_{p}= \begin{cases}1 & \text { for LKP sorption } \\ 0 & \text { for linear LIP sorption }\end{cases}
$$

In the case of LIP sorption, $K_{d}$ does not represent the distribution coefficient of LE sorption, but is rather a proportionality factor.

Thefirst-order reversiblechemicalsorptionisrepresentedbythelinearkineticchemical (LKC) model

$$
\frac{\partial F_{c}}{\partial t}+\lambda F_{c}=k_{c}^{+} K_{i} C-k_{c}^{-} F_{c}
$$


where $k_{c}^{+}\left[M^{-1} L^{3} T^{-1}\right]$ and $k_{c}^{-}\left[T^{-1}\right]$ are the forward and backward kinetic constants, respectively. Note that equation (11) can be used in conjunction with the physical sorption equations to describe combined sorption [Cameron and Klute, 1977], e.g., physical and chemical sorption. Combined sorption accounts for the different rates at which a species is sorbed onto different PM contituents. Thus, sorption onto organic components may be instantaneous(LE), whilesorptionontomineralsurfacesmaybemuchslowerandkinetically controlled [Cameron and Klute, 1977].

The equations of a series of $N_{c}$ first-order chemical reaction are given by [Cho, 1971]

$$
\begin{gathered}
\frac{\partial \mathcal{R}_{1}}{\partial t}=\mathcal{K}_{1} C_{1} \\
\frac{\partial \mathcal{R}_{2}}{\partial t}=\mathcal{K}_{2} C_{2}-\mathcal{K}_{1} C_{1} \\
\vdots \\
\frac{\partial \mathcal{R}_{N_{c}}}{\partial t}=\mathcal{K}_{N_{c}} C_{N_{c}}-\mathcal{K}_{N_{c}-1} C_{N_{c}-1}
\end{gathered}
$$

where $\mathcal{K}_{j}\left(j=1, \ldots, N_{c}\right)$ is the chemical reaction rate constant $\left[T^{-1}\right]$, and $N_{c}$ is the number of chemical reactions in the series.

\subsection{The Solute Transport ODE in the Laplace Space}

2.3.1. Parent or Stable Species. After incorporating the sorption terms, the Laplace transform (LT) of the solute transport equation (5) yields the following Ordinary Differential Equation (ODE)

$$
D \frac{d^{2} \widehat{C}}{d x^{2}}-U \frac{d \widehat{C}}{d x}-E \widehat{C}=0
$$

where $\widehat{C}=\mathcal{L}\{C\}, \mathcal{L}\{\}$ denotes the LT of the quantity in the brackets,

$$
E=\phi\left[\left(s+\delta_{\lambda} \lambda\right) R+\delta_{r} h \mathcal{K}\right]
$$




$$
R= \begin{cases}h+w \psi & \text { for LE sorption; } \\ h+u \psi & \text { for LKP or LIP sorption, } \\ h+v \psi & \text { for LKC sorption, } \\ h+(w+u) \psi & \text { for combined LE and LKP/LIP sorption, } \\ h+(w+v) \psi & \text { for combined LE and LKC sorption, } \\ h+(u+v) \psi & \text { for combined LKP/LIP and LKC sorption, }\end{cases}
$$

$$
D= \begin{cases}D_{T}+\phi \tau_{s} w \psi D_{s} & \text { for LE sorption; } \\ D_{T}+\phi \tau_{s} u \psi D_{s} & \text { for LKP or LIP sorption, } \\ D_{T}+\phi \tau_{s} v \psi D_{s} & \text { for LKC sorption, } \\ D_{T}+\phi \tau_{s}(w+u) \psi D_{s} & \text { for combined LE and LKP/LIP sorption, } \\ D_{T}+\phi \tau_{s}(w+v) \psi D_{s} & \text { for combined LE and LKC sorption, } \\ D_{T}+\phi \tau_{s}(u+v) \psi D_{s} & \text { for combined LKP/LIP and LKC sorption, }\end{cases}
$$

$$
w=K_{d} K_{i}, \quad u=\frac{k_{p} K_{d} K_{i}}{s+\lambda+\delta_{p} k_{p}}, \quad v=\frac{k_{c}^{+} K_{i}}{s+\lambda+k_{c}^{-}}, \quad \psi=\frac{(1-\phi)}{\phi} \rho
$$

and $s$ is the Laplace space parameter. The term $R$ is an expanded retardation factor, which can account for kinetic behavior [Moridis, 1999]. Its development involves the LT of the sorption from equations (8) through (11). It is straightforward to show that [Moridis, 1998]

$$
\widehat{F}=p \widehat{C}
$$


where $\widehat{F}=\mathcal{L}\{F\}$ and

$$
p= \begin{cases}w & \text { for LE sorption; } \\ u & \text { for LKP or LIP sorption, } \\ v & \text { for LKC sorption, } \\ w+u & \text { for combined LE and LKP/LIP sorption, } \\ w+v & \text { for combined LE and LKC sorption, } \\ u+v & \text { for combined LKP/LIP and LKC sorption. }\end{cases}
$$

Equation (13), subject to equations (14) through (19), is the ordinary differential equation (ODE) of solute transport in its most general form. Implicit in (13) are the assumptions that (a) $C(x, t=0)=0$, (b) $F(x, t=0)=0$, (c) $\mathcal{R}(x, t=0)=0$, and (d) in combined sorption, different sites are involved in each of the constituent types of sorption.

2.3.2. Daughter Species of Radioactive Decay. If the species is radioactive, the right-hand side of equation (5) is augmented by the term

$$
-\lambda_{\nu-1} m_{r}\left[\phi h C_{\nu-1}+(1-\phi) \rho F_{\nu-1}\right], \quad \text { where } \quad m_{r}=\frac{\mathcal{M}_{\nu}}{\mathcal{M}_{\nu-1}}
$$

$\mathcal{M}_{\nu}$ is the molecular weight of the $\nu$-th daughter $\left(1<\nu \leq N_{d}, N_{d}\right.$ being the total number of radioactive decay or reaction products), and $\nu-1$ refers to the decaying parent. Then, omitting for simplicitythe $n$ subscript, theLaplace spacetransport equation forany daughter product $\nu$ of the decay chain following a LE isotherm is given by

$$
D_{\nu} \frac{d^{2} \widehat{C}_{\nu}}{d x^{2}}-U \frac{d \widehat{C}_{\nu}}{d x}-E_{\nu} \widehat{C}_{\nu}=-G_{\nu} \widehat{C}_{\nu-1}
$$

where

$$
G_{\nu}=\phi m_{r} \lambda_{\nu-1} R_{\nu-1}
$$


If the daughter sorption is kinetically controlled, the kinetic sorption equations (9) and (11) need to account for the generation of daughter mass due to the decay of the sorbed parent, and become

$$
\frac{\partial F_{\nu}}{\partial t}+\lambda_{\nu} F_{\nu}-\lambda_{\nu-1} m_{r} \zeta_{\nu} F_{\nu-1}=k_{\alpha} C_{\nu}-k_{\beta} F_{\nu}
$$

where $F_{\nu-1}$ is the sorbed mass of the parent,

$$
k_{\alpha}=\left\{\begin{array}{ll}
k_{p} K_{d} K_{i} & \text { for LKP/LIP sorption, } \\
k_{c}^{+} K_{i} & \text { for LKC sorption, }
\end{array} \quad k_{\beta}= \begin{cases}k_{p} \delta_{p} & \text { for LKP/LIP sorption, } \\
k_{c}^{-} & \text {for LKC sorption, }\end{cases}\right.
$$

and $\zeta_{\nu}$ is the fraction of the mass of the decayed sorbed parent that remains sorbed as a daughter $\left(0 \leq \zeta_{\nu} \leq 1\right)$. The term $\zeta_{\nu}$ is introduced to account for the different sorption behavior of parents and daughters, and the fact that daughters can be ejected from grain surfaces due to recoil, e.g., the ejection of ${ }^{234}$ Th from grain surfaces during the alpha decay of ${ }^{238} \mathrm{U}$ [Faure, 1977]. The LT of (22) returns

$$
\widehat{F}_{\nu}=p \widehat{C}_{\nu}+m_{r} p_{r} \widehat{C}_{\nu-1}
$$

where $p$ is obtained from equation (19), and

$$
p_{r}= \begin{cases}\frac{\lambda_{\nu-1} \zeta_{\nu} u}{s+\lambda_{\nu}+k_{p} \delta_{p}} & \text { for (a) LKP/LIP or (b) combined LE-LKP/LIP sorption } \\ \frac{\lambda_{\nu-1} \zeta_{\nu} v}{s+\lambda_{\nu}+k_{c}^{-}} & \text {for (a) LKC sorption or (b) combined LE-LKC sorption }\end{cases}
$$

For combined LKC and LKP/LIP sorption, $p_{r}$ is the sum of the two components in (24). Using (23) and (24), it is easy to show that equation (20) applies, but with

$$
G_{\nu}=\phi m_{r}\left[\lambda_{\nu-1} R_{\nu-1}-\left(s+\lambda_{\nu}\right) p_{r}\right]
$$

All other terms in (20) remain unchanged. Equations (20) through (25) are valid in any layer $n$. It is obvious that for a complete daughter ejection, [Faure, 1977], $\zeta_{\nu}=0, p_{r}=0$, and (21) and (25) become identical. 
2.3.3. Products of Chemical Reactions. If the species is a product of the $\nu$-th firstorder chemical reaction in the reaction chain (12), the right-hand side of equation (5) is augmented by the term $-\phi h \mathcal{K}_{\nu-1} C_{\nu-1}$. Then, equation (20) applies unchanged, but with

$$
G_{\nu-1}=\phi h \mathcal{K}_{\nu-1}
$$

\section{Colloid Transport Equations}

\subsection{The PDE of Colloid Transport}

The 1-D PDE of transport of a radioactive or reactive true colloid (i.e., a colloid generated from contaminants when their concentrations exceed their solubility [Saltelli et al., 1984]) through a variably saturated PFM is described by equation (1) with the following changes:

(a) The term $C$ refers to the colloidal species. The term $C_{i}$ is entirely analogous, and equation (4) applies.

(b) Colloids do not support surface diffusion, thus $D_{s}=0$ in equation (3).

(c) The term $F$ is replaced by $\sigma$, which describes physical-chemical filtration of colloids (distinctly different from surface filtration and straining). Thus, the sorption term $(1-\phi) \rho \frac{\partial F}{\partial t}$ in equation (1) is replaced by the filtration term $\rho_{c} \frac{\partial \sigma}{\partial t}$, where $\rho_{c}$ is the colloid density $\left[M L^{-3}\right]$ and $\sigma$ is the filtered concentration of the colloid expressed as volume of colloids per volume of the porous medium.

(d) The velocity adjustment factor $f_{v}=1$ for solutes (see the definition after equation (1)), but $1 \leq f_{v} \leq 1.5$ in colloids. For $f_{v}>1$, it indicates that colloidal advection is larger than the average water velocity [Ibaraki and Sudicky, 1995]. This results from the relatively large size of the colloids, which leads to their concentration in the middle of the pores where the groundwater velocity is larger than the bulk average velocity. The factor $f_{v}$ tends to increase with decreasing ionic strength, but cannot exceed 1.5 
because colloids cannot move faster than the maximum groundwater velocity [Ibaraki and Sudicky, 1995].

(e) The dispersivities $\alpha_{L}$ and $\alpha_{T}$ are generally different from those for solutes [Ibaraki and Sudicky, 1995] and may be a function of the colloidal particle size.

(f) The term $D_{0}$ in $D_{m}$ and $D_{i}$ (equations (1) and (2)) is the colloidal diffusion coefficient in water $\left[L^{2} T^{-1}\right]$ and is described by the Stokes-Einstein equation as [Bird et al., 1960]

$$
D_{0}=\frac{k_{B} \mathcal{T}}{3 \pi \mu d_{c}}
$$

where $k_{B}$ is the Boltzmann constant( $1.38 \times 10^{-23} J K^{-1}$ inSIunits $), \mathcal{T}$ is the absolute water temperature $[K], \mu$ is the dynamic viscosity of water $\left[M L^{-1} T^{-1}\right]$, and $d_{c}$ is the colloid diameter $[L]$.

\subsection{The Equations of Colloid Filtration}

When colloid deposition is a relatively fast process compared to the water velocity, it is possible to describe colloid filtration as a linear equilibrium process [James and Chrysikopoulos, 1999]. Filtration is then described by

$$
\sigma=K_{\sigma} K_{i} C
$$

where $K_{\sigma}$ is a distribution coefficient $\left[M^{-1} L^{3}\right]$.

The colloid filtration is generally nonequilibrium and is more accurately described by a linear kinetic model [Çorapçioglu et al., 1987], which can take the following form:

$$
\frac{\partial \sigma}{\partial t}=\kappa\left(K_{\sigma} K_{i} C-\delta_{p} \sigma\right)=\kappa^{+} C-\kappa^{-} \sigma
$$

where $\kappa\left[T^{-1}\right]$ is a kinetic coefficient, and $\kappa^{+}\left[M^{-1} L^{3} T^{-1}\right]$ and $\kappa^{-}\left[T^{-1}\right]$ are the kinetic forward and reverse colloid deposition rates (clogging and declogging coefficients), 
respectively, whicharespecifictoeachcolloid and PFMtype. Theparameter $\delta_{p}$ is analogous to that for sorption in equation (10), and describes the reversibility of filtration.

The term $\kappa^{-}$is commonly assumed to be zero [Bowen and Epstein, 1979], but there is insufficient evidence to support this. The parameter $\kappa^{+}$can be given by

$$
\kappa^{+}=\kappa K_{\sigma} K_{i} \quad \text { or } \quad \kappa^{+}=\epsilon f_{v} U G
$$

where $\epsilon$ is the filter coefficient of the porous medium $\left[L^{-1}\right], f_{v}$ is a dimensionless velocity modification factor, $U$ is the Darcy velocity $\left[L T^{-1}\right]$, and $G$ is a dimensionless dynamic blocking function. (DBF) which describes the variation of the PFM porosity and specific surface with $\sigma$ [James and Chrysikopoulos, 1999]. For deep filtration (i.e., in the case of very dilute colloidal suspensions), there is no interaction among the colloidal particles and no effect on the medium porosity and permeability (i.e., $\phi$ is constant), and $G=1$.

The first expression in (30) is similar to that for linear kinetic sorption, and is an approximation that can be used effectively in studies where the water flow velocities vary within a narrow range. An example of such an application would be the study of colloid filtration in 1-D systems (columns) under steady-state flow conditions, from which the $\kappa^{+}$ and $\kappa^{-}$parameters can be determined. The second expression in (30) is more general, applies to domains in which the flow velocity varies within a wide range [de Marsily, 1986], and is conceptually more robust because it considers the effects of flow velocity on colloid attachment.

For transport through porous media or in a single fracture with fracture porosity $\phi \neq 1$, $\epsilon$ can be computed from Yao et al. [1971] or Tien et al. [1979] as

$$
\epsilon=1.5 \frac{1-\phi}{d_{m}} \eta_{c}
$$

where $d_{m}$ is the particle size of the medium grains or the fracture aperture $[L]$, and $\eta_{c}$ from Yaoet al. [1971] is 


$$
\eta_{c .}=\alpha_{c}\left[0.9\left(\frac{k_{B} T}{\mu d_{c} d_{m} U}\right)^{2 / 3}+1.5\left(\frac{d_{c}}{d_{m}}\right)^{2}+\left(\rho_{c}-\rho\right) \frac{g d_{c}^{2}}{18 \mu U}\right]
$$

in which $k_{B}$ is the Boltzman constant, $d_{C}$ is the colloid diameter $[L], \alpha_{C}$ is the singe collector efficiency, and all other terms remain as previously defined. Alternatively, $\eta_{c}$ in SI units can be computed from Tien et al. [1979] as

$$
\begin{aligned}
\eta_{c}=(1-\phi)^{2 / 3} A_{S} N_{L o}^{1 / 8} N_{R}^{15 / 8}+4 A_{S}^{1 / 3} N_{P e}^{-2 / 3} \\
+3.375 \times 10^{-3}(1-\phi)^{2 / 3} A_{S} N_{G}^{1.2} N_{R}^{-0.4}
\end{aligned}
$$

where

$$
\begin{gathered}
A_{S}=\frac{2\left(1-\omega^{5}\right)}{2-3 \omega+3 \omega^{5}-2 \omega^{6}}, \quad N_{L o}=\frac{4 \times 10^{-20}}{9 \pi \mu d_{c}^{2} U}, \quad N_{R}=\frac{d_{c}}{d_{m}} \\
N_{G}=\frac{\left(\rho_{c}-\rho\right) d_{c}^{2} g}{18 \pi \mu U}, \quad \cdot N_{P e}=\frac{3 \pi \mu d_{c} d_{m} U}{k T}, \quad \omega=(1-\phi)^{1 / 3} .
\end{gathered}
$$

\subsection{The Colloid Transport ODE in the Laplace Space}

The transport of a radioactive or reactive colloid through a variably saturated PFM in the Laplace space is described by equations (13) through (16) and (18) through (26) with the following changes:

(a) For colloids $D_{s}=0$ in equation (16).

(b) Equations (15), (16) and (19) reflect now colloid filtration that can be described by a linear equilibrium, linear kinetic, or linear irreversible relationship, or combinations thereof.

(c) The terms in equation (17) are now given by

$$
w=K_{\sigma} K_{i}, \quad u=\frac{\kappa^{+}}{s+\lambda+\kappa^{--}}, \quad v=0, \quad \text { and } \quad \psi=\frac{\rho_{c}}{\phi} .
$$

(d) In equation (22), $k_{\alpha}=\kappa^{+}$and $k_{\beta}=\kappa^{-}$, while the term $\zeta_{1}$ is the fraction of the mass of thefiltered (attached) parentcolloid that remains attached after thedecay $\left(0 \leq \zeta_{\nu} \leq 1\right)$. 
The term $p_{r}$ in (24) is now given by

$$
p_{r}=\frac{\lambda_{\nu-1} \zeta_{\nu}}{s+\lambda_{\nu}+\kappa^{-}} u
$$

\section{Transport in Layered Fractured Media}

The development of the equations for transport in a a layered fractured media expands on the analysis of Tang et al. [1981] and Sudicky and Frind [1982]. A schematic of the fracture-matrix system is shown in Figure 1, in which each of the $N$ layers has different properties.

\subsection{Transport in the Matrix}

4.1.1. The ODE of Parent or Stable Species Transport in the Matrix. Advection in the matrix is neglected, that is $U_{n}^{m}=0$. Then the Laplace space ODE of the species transport in the matrix layer $n$ is given by

$$
D_{n}^{m} \frac{d^{2} \widehat{C}_{n}^{m}}{d x_{n}^{2}}-E_{n}^{m} \widehat{C}_{n}^{m}=0
$$

where the $m$ superscript denotes the matrix. The diffusive flux across the fracture-matrix interface is given by

$$
q_{n}=-\left.r_{n} \chi_{n}^{m} D_{n}^{m} \frac{\partial C_{n}^{m}}{\partial x_{n}}\right|_{x_{n}=0}
$$

and differs from the analogous expression of Tang et al. [1981] in the inclusion of (a) the active interface area reduction factor $r_{n}$ and of (b) the accessibility factor $\chi_{n}$. The term $r_{n}$ $\left(1 \geq r_{n}>0\right)$ is defined as the ratio of the average interface area between mobile water in a fracture and its surrounding matrix to the average interface area between a fracture and the surrounding matrix. A detailed discussion on the subject can be found in Liu et al. [1998]. It is obvious that, for a fully saturated fracture, $r_{n}=1$. 
The accessibility factor $\chi_{n}^{m}$ adjusts the fracture-matrix interface fluxes within a layer; $\chi_{n}^{m}=1$ for solute transport, and $0 \leq \chi_{n}^{m} \leq 1$ for colloid transport. It describes the portion of the colloidal concentration in a medium allowed to enter an adjacent medium of different characteristics, and quantifies pore-size exclusion (straining).

4.1.2. The ODE of Daughter Transport in the Matrix. From equation (20), the Laplace space ODE of transport of the daughter $\nu$ in the matrix of layer $n$ is given by

$$
D_{n, \nu}^{m} \frac{d^{2} \widehat{C}_{n, \nu}^{m}}{d x_{n}^{2}}-E_{n, \nu}^{m} \widehat{C}_{n, \nu}^{m}=-G_{\nu}^{m} \widehat{C}_{n, \nu-1}^{m}
$$

where the term $G_{\nu}^{m}$ is computed from (21) to (26). The diffusive flux of the daughter $\nu$ across the fracture-matrix interface is given by equation (38).

\subsection{Transport in the Fractures}

4.2.1. Adjustments to Concepts and Equations. In fracture transport, the Darcy velocity $U_{n}$ in any layer $n$ is computed from the basic mass balance equation as

$$
U_{n}=\frac{Q_{w}}{M_{n} b_{n}}
$$

where $Q_{w}$ is the water influx rate per unit fracture thickness (in the $y$ direction, not shown in Figure 1) at the $z_{1}=0$ boundary $\left[L^{2} T^{-1}\right]$, and $2 b_{n}$ is the fracture aperture $[L]$. The parameter $M_{n}[L / L]$ is the fracture density, and is determined from the number of fractures inanarbitrary length $L_{x}$ (see Figure 1). Theterm $L_{x}$ is related tothe matrixblock half-width $X_{n}[L]$ and $b_{n}$ (see Figures $2 \mathrm{a}$ and $2 \mathrm{~b}$ ) through the relationship

$$
M_{n}=\frac{L_{x}}{2\left(X_{n}+b_{n}\right)}, \quad n=1, \ldots, N .
$$

There are two different ways to treat the fractures. If the fractures are open, we have surface-based rather than volume-based sorption in the fractures of any layer $n$ $(n=1, \ldots, N)$. The following changes are then made: 
(a) $F$ is now the mass of solute adsorbed per unit length of fracture surface, and has units $\left[M L^{-2}\right]$. Similarly, $\sigma$ is the volume of colloids attached per unit length of fracture surface, and has units of $\left[L^{-1}\right]$.

(b) From the mass balance equations, the term $(1-\phi) \rho$ in (17) and $\rho_{c}$ in equation (34) are replaced by $1 / b_{n}$, where $b_{n}$ is the fracture width $[L]$ in layer $n$.

(c) The distribution coefficient of the fracture $K_{d}^{f}$ is now defined as the mass of solute adsorbed per unit area of surface divided by the concentration of solute in solution [Tang et al., 1981], with units [L]. Similarly, the distribution coefficient of the fracture $K_{\sigma}^{f}$ is now defined as the mass of true colloids attached per unit area of surface divided by the concentration of colloids in suspension, with units $[L]$.

(d) The kinetic constants $k_{c}^{+}$of chemical sorption in (11) and $\kappa^{+}$in (30) have units $\left[M L^{-2} T^{-1}\right] ; k_{c}^{-}$in (11) and $\kappa^{-}$in (30) have units $\left[L T^{-1}\right]$. For transport through a single fracture with fracture porosity $\phi=1, \epsilon$ in (29) is now dimensionless and is given by Abdel-Salam and Chrysikopoulos [1995] as

$$
\epsilon=\frac{\eta_{d}}{2 b}
$$

where $\eta_{d}$ is the fracture surface colloid deposition coefficient $[L]$. Abdel-Salam and Chrysikopoulos [1995] and Chrysikopoulos and Abdel-Salam [1997] reported $\eta_{d}$ in the $10^{-10}-10^{-9} \mathrm{~m}$ range.

If the fractures are filled (a rather common occurrence), they are treated as a porous medium. Then, there is no need for the conceptual or mathematical adjustments in (1) through (4).

In both open and filled fractures, the right-hand side of equation (5) is augmented by the term

$$
Q_{n}=f_{n}^{q} q_{n}, \quad \text { where } f_{n}^{q}= \begin{cases}1 / b_{n} & \text { for open fractures } \\ 1 & \text { for filled fractures, }\end{cases}
$$

and $q_{n}$ is described by (38). 
4.2.2. The ODE of Parent or Stable Species Transport in the Fractures. The Laplacespaceequation forfracture transport alongthe $z$-coordinate(Figure 1)then becomes

$$
D_{n}^{f} \frac{d^{2} \widehat{C}_{n}^{f}}{d z_{n}^{2}}-U_{n} \frac{d \widehat{C}_{n}^{f}}{d z_{n}}-E_{n}^{f} \widehat{C}_{n}^{f}=\widehat{Q}_{n}
$$

where the $f$ superscript denotes the fracture, the $n$ subscripts denotes the layer, and $\widehat{Q}_{n}=\mathcal{L}\left\{Q_{n}\right\}$. Equation (42) is written in terms of the local coordinate $z_{n}$ in each layer $n$.

4.2.3. The ODE of Daughter Transport in the Fractures. The Laplace space ODE of transport for the daughter $\nu$ in the matrix of layer $n$ is given by

$$
D_{n, \nu}^{f} \frac{d^{2} \widehat{C}_{n, \nu}^{f}}{d z_{n}^{2}}-U_{n} \frac{d \widehat{C}_{n, \nu}^{f}}{d z_{n}}-E_{n, \nu}^{f} \widehat{C}_{n, \nu}^{f}=\widehat{Q}_{n}-G_{n}^{f} \widehat{C}_{n, \nu-1}^{f}
$$

All the terms in (43) are as previously defined.

\subsection{Initial and Boundary Conditions}

The initial and boundary conditions corresponding to the fracture equation are

$$
\begin{aligned}
& C_{n}^{f}\left(z_{n}, t=0\right)=0, \\
& C_{1}^{f}\left(z_{1}=0, t\right)=C_{z 0}(t), \\
& C_{n}^{f}\left(z_{n}=Z_{n}, t\right)=C_{n+1}^{f}\left(z_{n+1}=0, t\right), \quad n=1, \ldots, N-1, \\
& C_{N}^{f}\left(z_{N} \rightarrow \infty, t\right)=0,
\end{aligned}
$$

where $Z_{n}$ denotes the thickness of the n-th segment (layer). The time-dependence of $C_{z 0}$ allows investigation of systems with time-variable upper boundaries. Some of the more common forms of $C_{z 0}(t)$ are

$$
C_{z 0}(t)= \begin{cases}C_{0} & \text { constant concentration } \\ C_{0} \exp \left[-\lambda\left(t+t_{d}\right)\right] & \text { decaying radionuclide concentration } \\ \sum_{i=1}^{N^{*}} C_{i}^{*}\left[\mathcal{U}\left(t-t_{i-1}^{*}\right)-\mathcal{U}\left(t-t_{i}^{*}\right)\right] & \text { variable pulse concentration }\end{cases}
$$


where $C_{0}$ is a constant, $t_{d}$ is the release delay (the time between radionuclide generation or storage, and the beginning of release), $\mathcal{U}\left(t-t^{*}\right)$ denotes the unit step function at time $t^{*}$ (see Figure 3), and $N^{*}$ is the number of different concentration pulses (see Figure 3). Note that $t_{0}^{*}=0$ and that, for $N^{*}=1$, we obtain the unit pulse of duration $t_{1}^{*}$.

The initial and boundary conditions corresponding to the matrix equation are

$$
\begin{aligned}
& C_{n}^{m}(x, t=0)=0, \\
& C_{n}^{m}(x=0, t)=C_{n}^{f}\left(z_{n}, t\right), \\
& \left\{\begin{array}{cc}
\frac{\partial C_{n}^{m}}{\partial x}(x=X, t)=0 & \text { for Case 1 (Figure 2a), } \\
C_{n}^{m}(x \rightarrow \infty, t)=0 & \text { for Case 2 (Figure 2b), }
\end{array}\right.
\end{aligned}
$$

where $X$ is the half-width of the matrix block (Figure 2). Case 1 in Figure 2a describes a finite system with a Neuman boundary. If dry fractures (i.e., fractures in which the water phase is discontinuous) occur in the rock matrix of Case 1, the half-width $X$ is replaced by $X^{*}=2 X /\left(n_{d}+1\right)$, where $n_{d}$ is the number of dry fractures evenly spaced along $x$ in the matrix block (Figure $2 b$ ). Case 2 in Figure $2 \mathrm{~b}$ describes a semi-infinite system. The Laplace transforms of equations (44) through (46) are trivial.

\section{The Laplace Space Equations}

\subsection{General Matrix Solutions in Each Layer}

5.1.1. Parent or Stable Species. Omitting for simplicity the $n$ subscript, and expanding on Tang et al. [1981] and Sudicky and Frind [1982], the solutions to (37) are given by

$$
\widehat{C}^{m}= \begin{cases}H^{c} \cosh [\theta(X-x)] & \text { for Case 1 } \\ H^{e} \exp (-\theta x) & \text { for Case 2 }\end{cases}
$$

respectively, where $H^{c}$ and $H^{e}$ are parameters to be determined, and

$$
\theta=\theta(s)=\sqrt{\frac{E^{m}}{D^{m}}} .
$$


From (47) and the Laplace transform of (46),

$$
\widehat{C}^{m}(x=0)= \begin{cases}H^{c} \cosh (\theta X)=\widehat{C}^{f} \Rightarrow H^{c}=\frac{\widehat{C}^{f}}{\cosh (\theta X)} & \text { for Case 1 } \\ H^{e}=\widehat{C}^{f} & \text { for Case 2 }\end{cases}
$$

from which

$$
\widehat{C}^{m}=\widehat{C}^{m}(x, s)= \begin{cases}\frac{\cosh [\theta(X-x)]}{\cosh (\theta X)} \widehat{C}^{f} & \text { for Case } 1 \\ \exp (-\theta x) \widehat{C}^{f} & \text { for Case } 2\end{cases}
$$

Although the hyperbolic cosine solutions for $X \rightarrow \infty$ theoretically provides the same results with the exponential solution, in practice this is not the case because of difficulties in the computation of $\cosh [\theta(X-x)]$ for large values of the argument. The equations in (50) are applicable in any layer $n(n=1, \ldots, N)$.

5.1.2. Daughter or Reaction Products. Following the same approach, it is straightforward to show that the Laplace space solution of the ODE in (43) for any daughter or reaction product $\nu$ is given by

$$
\widehat{C}_{\nu}^{m}= \begin{cases}H_{\nu}^{c} \cosh \left[\theta_{\nu}(X-x)\right]+\sum_{\kappa=\nu-1}^{1}\left(\prod_{i=\nu}^{\kappa} A_{i \kappa}^{m}\right) H_{\kappa}^{c} \cosh \left[\theta_{\kappa}(X-x)\right] & \text { for Case 1 } \\ H_{\nu}^{e} \exp \left(-\theta_{\nu} x\right)+\sum_{\kappa=\nu-1}^{1}\left(\prod_{i=\nu}^{\kappa} A_{i \kappa}^{m}\right) H_{\kappa}^{e} \exp \left(-\theta_{\kappa} x\right) & \text { for Case 2 }\end{cases}
$$

where

$$
A_{i \kappa}^{m}=-\frac{G_{i}^{m}}{D_{i}^{m} \theta_{\kappa}^{2}-E_{i}^{m}} .
$$

The coefficients $H_{\nu}$ are given by the general expression

$$
H_{\nu}=\sum_{\kappa=1}^{\nu} T_{\nu, \kappa} \widehat{C}_{\kappa}^{f},
$$

where $T_{\nu, \kappa}$ are appropriate coefficients. Expressions for $H_{\nu}$ and $T_{\nu, \kappa}$ (the derivation of which is tedious but straightforward) for Cases 1 and 2 are provided in Appendix A. 
Equation (51) shows that the solution of the matrix transport equation of the daughter or reaction product $\nu$ requires knowledge of the fracture solutions of all previous members of the decay or reaction chain.

\subsection{General Fracture Solutions in Each Layer}

5.2.1. Parent or Stable Species. From the Laplace transform of the diffusive flux in (42), and omitting for simplicity the subscript $n$,

$$
\widehat{Q}=\gamma f^{q} \widehat{C}^{f}
$$

where

$$
\gamma= \begin{cases}r \chi^{m} D^{m} \theta \tanh (\theta X) & \text { for Case } 1 \\ r \chi^{m} D^{m} \theta & \text { for Case 2 }\end{cases}
$$

Substituting in (42) and collecting terms,

$$
D^{f} \frac{d^{2} \widehat{C}^{f}}{d z^{2}}-U \frac{d \widehat{C}^{f}}{d z}-E^{*} \widehat{C}^{f}=0
$$

where $E^{*}=E^{f}+\gamma f^{q}$. The general solution to (56) is given by

$$
\widehat{C}^{f}=\widehat{C}^{f}(x, s)=\alpha \exp \left(\eta^{+} z\right)+\beta \exp \left(\eta^{-} z\right)
$$

where $\alpha$ and $\beta$ are parameters to be determined, and

$$
\eta^{ \pm}=\frac{U \pm \sqrt{U^{2}+4 D^{f} E^{*}}}{2 D^{f}}
$$

Equations (54)-(58) apply in any layer $n$.

5.2.2. Daughter or Reaction Products. From equations (41)-(44) and (51)-(53), for a daughter $\nu$

$$
\widehat{Q}_{\nu}=f^{q} r \chi^{m} D_{\nu}^{m} W_{\nu}=f^{q} \sum_{\kappa=1}^{\nu} \gamma_{\nu, \kappa} \widehat{C}_{\kappa}^{f}
$$


Equation (59) is general and applies to both Case 1 and Case 2. Expressions for $W_{\nu}$ and $\gamma_{\nu, \kappa}$ (the derivation of which is tedious but straightforward) for Cases 1 and 2 are provided in Appendix B.

Substituting in (43) and collecting terms,

$$
D_{\nu}^{f} \frac{d^{2} \widehat{C}_{\nu}^{f}}{d z^{2}}-U \frac{d \widehat{C}_{\nu}^{f}}{d z}-E_{\nu}^{*} \widehat{C}_{\nu}^{f}=-G_{\nu} \widehat{C}_{\nu-1}^{f}+f^{q} \sum_{\kappa=1}^{\nu-1} \gamma_{\nu, \kappa} \widehat{C}_{\kappa}^{f}
$$

where $E_{\nu}^{*}=E_{\nu}^{f}+\gamma_{\nu, \nu} f^{q}$

Following the same approach, it is straightforward to show that the Laplace space solution of any daughter or reaction product $\nu$ is given by

$$
\widehat{C}_{\nu}^{f}=\alpha_{\nu} \exp \left(\eta_{\nu}^{+} z\right)+\beta_{\nu} \exp \left(\eta_{\nu}^{-} z\right)+Y_{\nu}
$$

where

$$
Y_{\nu}=\sum_{\kappa=\nu-1}^{1} A_{\nu, \kappa}^{+} \alpha_{\kappa} \exp \left(\eta_{\kappa}^{+} z\right)+\sum_{\kappa=\nu-1}^{1} A_{\nu, \kappa}^{-} \beta_{\kappa} \exp \left(\eta_{\kappa}^{-} z\right)
$$

and

$$
A_{\nu, \kappa}^{ \pm}=\frac{B_{\nu, \kappa}^{ \pm}}{D_{\nu}^{f}\left(\eta_{\kappa}^{ \pm}\right)^{2}-U \eta_{\kappa}^{ \pm}-E_{\nu}^{*}} .
$$

The computation of the $B_{\nu, \kappa}^{ \pm}$coefficients is tedious but straightforward. Expressions for $B_{\nu, \kappa}^{ \pm}$and for $\nu \leq 5$ are given in Appendix C. Equations (61) and (62) show that the solution of the fracture transport equation of the daughter or reaction product $\nu$ requires knowledge of all previous $\alpha_{\nu}$ and $\beta_{\nu}$, i.e., the solutions of all previous members of the decay or reaction chain.

\section{The Solution Approach}

\subsection{Determination of the $\alpha$ and $\beta$ Parameters}

Equation (57) defines a total of $2 N$ unknowns, i.e., the $\alpha$ and $\beta$ parameters in each of the $N$ subdomains. These are obtained from the solution of the following equations. 
6.1.1. Boundary Equations. These apply to the $z_{1}=0$ point in the first layer $(n=1)$. From (57) and the Laplace transform of (44), for a known boundary concentration we have

$$
\alpha_{1}+\beta_{1}=\widehat{C}_{z 0}
$$

while for known flux boundary conditions

$$
\alpha_{1}\left(U_{1}-D_{1}^{f} \eta^{+}\right)+\beta_{1}\left(U_{1}-D_{1}^{f} \eta^{-}\right)=U_{1} \widehat{C}_{z 0}
$$

where $\widehat{C}_{z 0}=\mathcal{L}\left\{C_{z 0}\right\}$. For the common boundary conditions in (45),

$$
\widehat{C}_{z 0}= \begin{cases}\frac{C_{0}}{s} & \text { constant concentration } \\ \frac{C_{0} \exp \left(-\lambda t_{d}\right)}{s+\lambda} & \text { decaying radionuclide concentration } \\ \sum_{i=1}^{N^{*}} \frac{C_{i}^{*}}{s}\left[\exp \left(-s t_{i-1}^{*}\right)-\exp \left(-s t_{i}^{*}\right)\right] & \text { piecewise constant concentration. }\end{cases}
$$

For the limiting case of a system consisting of a single semi-infinite layer (i.e., $N=1$ ) with an open fracture and a constant concentration at $z_{1}=0, \alpha_{1}=0, \beta_{1}=C_{0} / s$, and equation (57) is reduced to the Laplace space solutions obtained by Tang et al. [1981] (Case 2) and Sudicky and Frind [1982] (Case 1).

6.1.2. Concentration Equations. At the layer interfaces we have the equations

$$
\alpha_{n-1} \exp \left(\eta_{n-1}^{+} Z_{n-1}\right)+\beta_{n-1} \exp \left(\eta_{n-1}^{-} Z_{n-1}\right)-\alpha_{n}-\beta_{n}=0
$$

for $n=2, \ldots, N$. An additional equation is provided by the requirement that $\widehat{C}_{n}^{f}$ be finite for $Z_{N} \rightarrow \infty$, which dictates that $\alpha_{N}=0$.

6.1.3. Flux Equations. The remaining $N-1$ equations are provided by the equality of fluxes across the layer boundaries in the fractures, which dictates that

$$
M_{n-1} b_{n-1}\left[U_{n-1} C_{n-1}^{f}-D_{n-1}^{f} \frac{d C_{n-1}^{f}}{d z_{n-1}}\right]_{Z_{n-1}}=M_{n} b_{n}\left[U_{n} C_{n}^{f}-D_{n}^{f} \frac{d C_{n}^{f}}{d z_{n}}\right]_{0},
$$


in which the quantity in the brackets is computed at the value of the local $z$ coordinate indicated by the bracket subscript. From (57) and (68) we obtain

$$
\begin{aligned}
& \alpha_{n-1}\left[M_{n-1} b_{n-1}\left(U_{n-1}-D_{n-1}^{f} \eta_{n-1}^{+}\right)\right] \exp \left(\eta_{n-1}^{+} Z_{n-1}\right) \\
& +\beta_{n-1}\left[M_{n-1} b_{n-1}\left(U_{n-1}-D_{n-1}^{f} \eta_{n-1}^{-}\right)\right] \exp \left(\eta_{n-1}^{-} Z_{n-1}\right) \\
& \quad-\alpha_{n}\left[M_{n} b_{n}\left(U_{n}-D_{n}^{f} \eta_{n}^{+}\right)\right]-\beta_{n}\left[M_{n} b_{n}\left(U_{n}-D_{n}^{f} \eta_{n}^{-}\right)\right]=0
\end{aligned}
$$

where $n=1, \ldots, N-1$.

6.1.4. Equations for Daughters. For a daughter product $\nu$ of radioactive decay or reaction, the following changes are made to equations (64) through (69):

(a) In the right-hand side of equations (64) and (65), the term $\widehat{C}_{z 0}$ is replaced by $\widehat{C}_{\nu, z 0}$, where $\widehat{C}_{\nu, z 0}=\mathcal{L}\left\{C_{\nu, z 0}\right\}$, and $C_{\nu, z 0}$ is the concentration of daughter $\nu$ at the $z_{1}=0$ boundary. For a constant $C_{\nu, z 0}, \widehat{C}_{\nu, z 0}$ can be obtained from equation (66). For a $z_{1}=0$ boundary with a decaying radionuclide concentration, $\widehat{C}_{\nu, z 0}$ is computed from theLaplace transform ofthe mass balance equation $\frac{\partial C_{\nu, z 0}}{\partial t}=\lambda_{\nu} C_{\nu, z 0}-\lambda_{\nu-1} C_{\nu-1, z 0}$ as

$$
\widehat{C}_{\nu, z 0}=\frac{C_{\nu, z 0}}{s+\lambda_{\nu}} \exp \left(-\lambda t_{d}\right)+m_{r} \frac{\lambda_{\nu-1}}{s+\lambda_{\nu}} \exp \left(-\lambda t_{d}\right) \widehat{C}_{\nu-1, z 0}
$$

For a reaction chain, equation (70) indicates a recursive reaction.

(b) The 0 on the right-hand side of the layer interface equation (67) is replaced by $Y_{\nu, n}\left(z_{n}=0\right)-Y_{\nu, n-1}\left(z_{n-1}=Z_{n}\right)$ for $n=2, \ldots, N$.

(c) Equation (68) applies unchanged. The 0 on the right-hand side of equation (69) is replaced by the known quantity

$$
\begin{aligned}
M_{n} b_{n}\left[U_{n} Y_{\nu, n}\right. & \left.-D_{\nu, n}^{f} \frac{d Y_{\nu, n}}{d z_{n}}\right]_{0} \\
& -M_{n-1} b_{n-1}\left[U_{n-1} Y_{\nu, n-1}-D_{\nu, n-1}^{f} \frac{d Y_{\nu, n-1}}{d z_{n-1}}\right]_{Z_{n-1}}
\end{aligned}
$$

6.1.5. Parent-Daughter Species. Note that the development of the equations for parents and daughters is general and does notrestrict radioactive chains tosolute or colloidal 
species. Thus, colloidal parents can have solute daughters if the daughter solubility exceeds that of the parent (a distinct possibility in the transport of small-diameter colloids). The reverse is also possible if the parent solubility exceeds that of the daughter.

\subsection{The Laplace Space Solutions}

The generality and complexity of these equations preclude the development of closedform solutions for $\alpha_{i}, \beta_{i}(i=1, \ldots, N)$. Consequently, it is not possible to analytically invert equations (57) or (61), and to obtain a closed-form equation for concentration in time. The problem is alleviated by numerically inverting the Laplace space solutions. The algebraic equations discussed in Section 6.1 may be written in a general matrix form as:

$$
\mathbf{M} \overrightarrow{\mathbf{X}}=\overrightarrow{\mathbf{B}}
$$

where $\mathbf{M}$ is the coefficient matrix, $\overrightarrow{\mathbf{X}}$ is the vector of the unknowns, and $\overrightarrow{\mathbf{B}}$ is the composite vector of knowns. Solution of (71) returns the vector

$$
\overrightarrow{\mathbf{X}}=\left(\begin{array}{c}
\overrightarrow{\mathbf{X}}_{1} \\
\overrightarrow{\mathbf{X}}_{2} \\
\vdots \\
\overrightarrow{\mathbf{X}}_{N}
\end{array}\right), \quad \text { where } \quad \overrightarrow{\mathbf{X}}_{i}=\left(\begin{array}{c}
\alpha_{i} \\
\beta_{i}
\end{array}\right), i=1, \ldots, N
$$

The solution of the matrix equation (71) necessitates arithmetic values for the $s$ parameter of the Laplace space. These are provided by the numerical inversion scheme of DeHoog et al. [1982] that uses complex values for $s$. A detailed discussion of the application of this method and its performance can be found in Sudicky [1990] and Moridis [1998]. The quantities $\mathbf{M}, \overrightarrow{\mathbf{X}}$ and $\overrightarrow{\mathbf{B}}$ assume the complex type of $s$.

The $\alpha_{i}$ and $\beta_{i}$ computed from the matrix equation (71) are then used to obtain all the $\widehat{C}_{n}^{f}$ solutions $(i=1, \ldots, N)$. The corresponding $\widehat{C}_{n}^{m}$ solutions are obtained from $\widehat{C}_{n}^{f}$ and equations (50) or (51)-(53). Note that the solutions for daughters or reaction products 
requires knowledge of the solutionsof all the previous membersinthe radioactive or reactive chain.

\subsection{Numerical Inversions of the Laplace Space Solutions}

The various time-variable concentrations can be determined by numerically inverting the Laplace space solutions, i.e.,

$$
C_{n}^{f}(x, t)=\mathcal{L}^{-1}\left\{\widehat{C}_{n}^{f}(x, s)\right\}, \quad C_{n}^{m}(x, t)=\mathcal{L}^{-1}\left\{\widehat{C}_{n}^{m}(x, s)\right\}
$$

where $\mathcal{L}^{-1}\{\}$ denotes the inverse Laplace transform of the quantity in the brackets. Details on the inversion will not be discussed here; they can be found in DeHoog et al. [1982] and in Moridis [1998].

\section{Treatment of Special Conditions}

\subsection{Colloid Transport With Straining}

Ifstraining acrossthe interfaces(fracture-fracture or fracture-porousmedium) of layers is considered, the determination of the $\alpha$ and $\beta$ parameters is more complicated and can involve several stages. The solution process is as follows:

(a) The system described by equations (64)-(69) is solved, and $\alpha_{n}, \beta_{n}(n=1, \ldots, N)$ are computed.

(b) The potential (maximum) flux at the bottom boundary of the first layer (first interface) is computed as

$$
\begin{aligned}
\widehat{Q}_{Z_{1}} & =\left[U_{1} \widehat{C}_{1}^{f}-D_{1}^{f} \frac{d \widehat{C}_{1}^{f}}{d z_{1}}\right]_{z_{1}=Z_{1}} \\
& =\alpha_{1}\left(U_{1}-D_{1}^{f} \eta_{1}^{+}\right) \exp \left(\eta_{1}^{+} Z_{1}\right)+\beta_{1}\left(U_{1}-D_{1}^{f} \eta_{1}^{-}\right) \exp \left(\eta_{1}^{-} Z_{1}\right)
\end{aligned}
$$

This represents the Laplace-transformed flux if all the colloids can move uninhibited (i.e., without straining) across the layer interface. Because all the quantities in (74) are known, $\widehat{Q}_{Z_{1}}$ is easily computed. 
(c) The actual transformed flux across the straining boundary is now

$$
\begin{aligned}
\widehat{Q}_{Z_{1}}^{*} & =\alpha_{1}^{*}\left(U_{1}-D_{1}^{f} \eta_{1}^{+}\right) \exp \left(\eta_{1}^{+} Z_{1}\right)+\beta_{1}^{*}\left(U_{1}-D_{1}^{f} \eta_{1}^{-}\right) \exp \left(\eta_{1}^{-} Z_{1}\right) \\
& =\chi_{2}^{f} \widehat{Q}_{Z_{1}}
\end{aligned}
$$

where the superscript ${ }^{*}$ denotes the actual (as opposed to potential) values, and $\chi_{2}^{f}$ is a dimensionless accessibility factor [Moridis et al., 1999]. The term $\chi_{2}^{f}$ adjusts the fluxes at the fracture interfaces of layers 1 and 2. It describes the portion of the colloidal concentration in the fractures that is allowed to enter the fractures (or, in the case of an unfractured layer, the flowing portion of the matrix) of an adjacent layer of different characteristics, and quantifies pore size exclusion (straining). For solutes $\chi_{2}^{f}=1$, while for colloids $0 \leq \chi_{2}^{f} \leq 1$ because of their relatively large size.

Forthefirst layer $(n=1)$, equations(64)-(66)areunaffected. Equation (67)is replaced by the actual flux equation (75). The right hand side $\left(=\chi_{2}^{f} \widehat{Q}_{Z_{1}}\right)$ and the coefficients of $\alpha_{1}^{*}$ and $\beta_{1}^{*}$ in (75) are known quantities.

(d) The flux equation (69) for $n=2$ now becomes

$$
\begin{aligned}
& \alpha_{1}^{*}\left[M_{1} b_{1}\left(U_{1}-D_{1}^{f} \eta_{1}^{+}\right)\right] \exp \left(\eta_{1}^{+} Z_{1}\right) \\
& +\beta_{1}^{*}\left[M_{1} b_{1}\left(U_{1}-D_{1}^{f} \eta_{1}^{-}\right)\right] \exp \left(\eta_{1}^{-} Z_{1}\right) \\
& \quad-\alpha_{2}\left[M_{2} b_{2}\left(U_{2}-D_{2}^{f} \eta_{2}^{+}\right)\right]-\beta_{2}\left[M_{2} b_{2}\left(U_{2}-D_{2}^{f} \eta_{2}^{-}\right)\right]=0 .
\end{aligned}
$$

(e) Equations (67) and (69) apply for $n>2$. The resulting system of linear equations in (71) is solved again to yield $\alpha_{1}^{*}, \beta_{1}^{*}, \alpha_{n}, \beta_{n}(n=2, \ldots, N)$.

(f) The potential (maximum) flux $\widehat{Q}_{Z_{2}}$ at the bottom boundary of the next layer $n=2$ is then computed in analogously to that in equation (74). In the same layer, equation (67) is replaced by the equation of the corrected flux in (75), and the adjusted flux at the boundary between the $n=2$ and $n=3$ layers is obtained from (76) after the subscript substitutions $2 \rightarrow 1$ and $3 \rightarrow 2$. The new system of linear equations (71) is solved again to yield $\alpha_{1}^{*}, \beta_{1}^{*}, \alpha_{2}^{*}, \beta_{2}^{*}, \alpha_{n}, \beta_{n}(n=3, \ldots, N)$. 
(g) The process is repeated until all the concentration equations (67) are replaced by the corrected flux equations (75). The solution of the linear system (71) then returns $\alpha_{n}^{*}$, $\beta_{n}^{*}(n=1, \ldots, N)$. Using the $\alpha_{n}^{*}$ and $\beta_{n}^{*}$ values (instead of $\alpha_{n}$ and $\beta_{n}$ ) in (57) yields $C_{n}^{f}$.

It is obvious that colloid filtration with pore-size exclusion (straining) requires solution of the linear system (71) a maximum of $N$ times. Note that there is no need to apply the process described above at the interfaces where no straining occurs.

Extension to daughter products (colloidal or solute) of the decay of radioactive colloids is straightforward. Equations (65)-(69), as modified per the discussion in Section 6.1.4, apply to the initial (unstrained) system. The quantity

$$
\left[U_{1} Y_{\nu, 1}-D_{\nu, 1}^{f} \frac{d Y_{\nu, 1}}{d z_{1}}\right]_{Z_{1}}
$$

is added to the expression for $\widehat{Q}_{Z_{1}}$ in the second line of (74), whereas the quantity

$$
\left[U_{1} Y_{\nu, 1}^{*}-D_{\nu, 1}^{f} \frac{d Y_{\nu, 1}^{*}}{d z_{1}}\right]_{Z_{1}}
$$

is subtracted from the right-hand side (second line) of (75), where $Y^{*}$ indicates particular solutions based on the corrected fluxes.

\subsection{Misaligned Fractures}

The analysis presented thus far assumes that the effect of fracture offset on transport is negligible. This may not be the case for large fracture spacing or at short observation times.

The process that accounts for fracture misalignment is described in Figure 4. The increased travel path of the transporting water caused by the offset fractures is indicated by the horizontal pathway at the confluence of the $n$ and $n+1$ layers in Figure $4 \mathrm{a}$, and its effect is described by the addition of an "interlayer", i.e., a pseudo-layer (Figure 4b) with the following characteristics: 
(a) A thickness $Z_{I}=\max \left\{X_{n}, X_{n+1}\right\}$ when $M_{n}>M_{n+1}$, or $Z_{I}=\min \left\{X_{n}, X_{n+1}\right\}$ when $M_{n}<M_{n+1}$.

(b) A relative frequency $M_{I}=M_{n}$.

(c) An open or filled fracture of width $b_{I}$ through which water flows between the $n$ and $n+1$ layers. The properties of the fracture in the interlayer are independent of those in the layers above and below.

(d) A complex matrix, composed of the matrices of both the $n$ and $n+1$ layers. In Figure $4 \mathrm{~b}$, the matrices of the $n$ and $n+1$ layers are positioned on the left and right sides of the fracture, respectively. The two components of the matrix are assumed to be semi-infinite, as illustrated by their rotation by $90^{\circ}$ (with respect to the original layer orientation) in Figure 4b. Then, the flux into the composite matrix of the interlayer is computed from equation (54), but with $\gamma \equiv \gamma_{I}$, where

$$
\gamma_{I}=\frac{1}{2}\left(\gamma_{n}+\gamma_{n+1}\right)
$$

and $\gamma_{n}, \gamma_{n+1}$ are computed from equation (55).

Thus, considerationof misaligned fracturestransformsasystem of $N$ layerstoasystem of $N+N_{I}$ layers, where $N_{I}$ is the number of interlayers. The solution of the augmented system does not pose any particular challenges and proceeds in the manner discussed in Section 6. Note that the approximation discussed involves the longest possible travel path and the largest possible amount of tracer diffusing into the matrix. This is because the concept of the interlayer assumes semi-infinite pseudomatrices (see Figure $4 \mathrm{~b}$ ) and ignores cross diffusion between the $n$ and $n+1$ layers. In that respect, the transport estimates considering and ignoring fracture offset provide the limits that bracket the true solution.

\subsection{Occasional Unfractured Layers}

If the layered system includes unfractured (porous) layers (e.g., Layer 3 in Figure 
1), these are treated as a combination of a pseudo-matrix (representing the nonflowing portion of the layer) and a pseudo-fracture representing the flowing portion of the layer. In essence, unfractured layers are treated as filled-fracture systems, and all the equations apply unchanged. The properties of the unfractured medium are assigned to both the pseudomatrix and the pseudo-fracture. The relative sizes of $b$ and $X$ can describe the flowing and non-flowing portions of the porous medium. If water flows uniformly through the porous medium, $X=0$. This approach maintains water mass and flux balance.

It is obvious that, for unfractured media, $L_{x}=2\left(b_{n}+X_{n}\right)$, i.e., $M_{n}=1$. Note that correct water saturations $S$ must be used (obtained from the solution of the steady-state flow equation) because the derivation of the transport equations is based on time-invariant flow conditions and cannot compute changes in $S$.

\subsection{Transport in Layered Unfractured Media}

This is a limiting case of the scenario discussed in Section 7.3. Setting $X_{n}=0$ $(n=1, \ldots, N)$ transforms the problem into that of one-dimensional tracer transport in a layered porous (unfractured) system. Then, all the semianalytical solutions derived here apply unchanged.

\section{Verification}

A FORTRAN program was written to obtain the semianalytical (SA) solutions developed in Sections 4 through 6 by first solving (71), and then performing the numerical inversion indicated in (73). This code, named FRACL, accounts for all the processes, phenomena and conditions discussed in Sections 2 through 7. It can obtain solutions for a system involving an arbitrary number of layers $N$ of any combination of porous and/or fractured media, and up to 4 daughters (i.e., 5 radionuclides, including the parent). It is 
very computationally efficient, and required less than 10 seconds for any of the problems discussed in Sections 8 or 9.

FRACL is verified through comparisons to analytical solutions of radioactive solute and colloid transport in 1-D porous (unfractured) media and 2-D fractured media. In all cases, FRACL solutions are first obtained in a system consisting of a single semi-infinite layer (i.e., $N=1$ ). The domain is then subdivided into three layers in the $z$ direction, and FRACL solutions for this multilayered system $(N=3)$ are obtained. Coincidence of the analytical solutions to the FRACL solutions for $N=1$ and for $N=3$ validates FRACL.

\subsection{Test FS1: Radioactive Solute Transport in a System of Parallel Fractures}

This problem describes transport with LE sorption in the fracture-matrix system of Case 1 (Figure 2a). The corresponding analytical solution was developed by Sudicky and Frind [1982]. The values of the parameters used for the computation of the analytical and the SA solutions are as in Sudicky and Frind [1982], and are listed in Table 1. A constant concentration condition is applied at $z_{1}=0$.

Figure 5 shows the distribution of the relative concentration $C_{R}$ (defined as $C_{R}=$ $\left.C_{n}^{f} / C_{z 0}\right)$ in the fractures along the $z$ axis at $t=1,000$ days. The analytical solution and the two FRACL solutions (for $N=1$ and $N=3$ ) are identical in the first 5 significant digits.

\subsection{Test FS2: Radioactive Solute Transport in a Single Fracture}

This problem describes transport with LE sorption in the fracture-matrix of Case 2 (Figure 2b). The corresponding analytical solution was developed by Tang et al. [1981]. The values of the parameters used for the computation of the analytical and the SA solutions are the same as in Test FS1, and are listed in Table 1. A constant concentration condition is applied at $z_{1}=0$. 
Figure 6 shows the distribution of $C_{R}$ in the fracture along the $z$ axis at $t=10,000$ days. The analytical solution and the two FRACL solutions (for $N=1$ and $N=3$ ) are identical in the first five significant digits.

\subsection{Tests FS3 and FS4: Transport of a Two-Member Radioactive Solute Chain in a Single Fracture and in Parallel Fractures}

To verify the ability of FRACL to predict the transport of the members of radioactive/reactive chains, the transport of a parent and a single daughter is studied in Tests FS3 and FS4 (corresponding to theconditions of Case 1 and 2, respectively). Theparent radionuclide is non sorbing and has a short half life $\left(t_{1 / 2}=1,000 \mathrm{~s}\right)$. The daughter radionuclide has the properties of the radionuclide discussed in Tests FS1 and FS2 (Table 1). The boundary conditions and the values of the parameters are the same as in Tests FS1 and FS2. The observation time in this test is long ( $t=100,000$ days). The very short $T_{1 / 2}$ of the parent ensures complete decay to the daughter radionuclide at the time of observation.

The SA solutions of Tests FS3 and FS4 for $N=1$ and $N=3$ are shown in Figure 7. The SA predictions of the $C_{R}$ distributions in the fractures for both $N=1$ and $N=3$ coincide with the analytical solutions of Sudicky and Frind [1982] and Tang et al. [1981].

An analytical solution to the problem of transport of a parent and a single daughter was developed by Sudicky and Frind [1984], but was based on a simplified PDE of fracture transport that neglects dispersion and involving a delta-Dirac type of contaminant release. Because of these differences, it is not possible to compare the SA solution from FRACL to the Sudicky and Frind [1984] solution.

\subsection{Tests FC1 and FC2: Colloid Transport in a Single Fracture}

These tests correspond to nonradioactive colloid transport with kinetic filtration (deposition) in the fracture-matrix system of Case 2 (Figure 2b). Abdel-Salam and 
Chrysikopoulos [1994] developed the corresponding analytical solutions for different boundary conditions and matrix penetration scenarios.

The values of the parameters used for the computation of the analytical and the SA solutions of Tests FC1 and FC2 are listed in Table 2. In both tests, a constant flux condition is applied at $z_{1}=0$. Filtration in the matrix is not considered in Test FC1, while matrix deposition is controlled by a linear kinetic model in Test FC2.

Figure 8 shows the distribution of the relative concentration $C_{R}$ in the fractures along the $z$ axis at $t=5$ years. The SA predictions of $C_{R}$ in the fractures for both $N=1$ and $N=3$ coincide with the analytical solutions of Abdel-Salam and Chrysikopoulos [1997].

\subsection{Tests PS1 to PS4: Radioactive Solute Transport in Unfractured Porous Media}

Tests PS1 to PS4 are designed to confirm the ability of the SA solutions to describe transport in unfractured media without any modification. The solution to this problem is provided by Bear [1979], and accounts for LE sorption and radioactive decay.

The values of the parameters used for the computation of the analytical and the SA solutions of Tests PS1 to PS4 are listed in Table 3. In all four tests, a constant concentration condition is applied at $z_{1}=0$. The solute is a nondecaying isotope in Tests PS1 and PS2, and a decaying radionuclide in Tests PS3 and PS4. LE sorption is considered in Tests PS2 and PS3, but is ignored in Tests PS1 and PS4.

Figure 9 shows the distribution of the relative concentration $C_{R}$ along the $z$ axis at $t=200$ days. The SA predictions of $C_{R}$ distributions for both $N=1$ and $N=3$ are identical with the analytical solutions of Bear [1979].

\subsection{Test PS5: Transport of a Three-Member Radioactive Solute Chain in Unfractured Porous Media}


This test is designed to verify the ability of the SA solutions to describe the transport of reactive chains in unfractured media without any modification. An analytical solution to this problem was developed by Harada et al. [1980], and accounts for LE sorption, radioactive decay, and time-variable boundary conditions.

Test PS5 describes the transport of the radioactive chain

$$
{ }^{234} \mathrm{U} \longrightarrow{ }^{230} \mathrm{Th} \longrightarrow{ }^{226} \mathrm{Ra}
$$

through a sorbing porous medium. The concentration of ${ }^{234} \mathrm{U}$ (i.e., the parent radionuclide) at the $z_{1}=0$ is not constant over time, but subject to radioactive decay. The initial concentrations of the ${ }^{230} \mathrm{Th}$ and ${ }^{226} \mathrm{Ra}$ daughter radionuclides at the $z_{1}=0$ boundary are zero, but increase over time because of the decay of their parents.

The values of the parameters used for the computation of the analytical and the SA solutions of Test PS5 are as in Harada et al. [1980], and are listed in Table 4. Figure 10 shows that the analytical solutions at $t=10,000$ years coincide with the SA predictions (for both $N=1$ and $N=3$ ) of the $C_{R}$ distributions of the three members of the radioactive chain.

\subsection{Tests PC1 to PC3: Transport of Non-Radioactive Colloids in Unfractured Porous Media}

Tests PC1 to PC3 describe nonradioactive colloid transport in a porous medium under conditions of "deep filtration" (see Section 3.2). An analytical solution to this problem for $f_{v}=1$ (see Sections 3.1 and 3.2) was developed by Dieulin [1982], and accounts for kinetic irreversible filtration (i.e., $\kappa^{-}=0$ in equation (29)).

The values of the parameters used for the computation of the analytical and the SA solutions of Tests PC1 to FC3 are listed in Table 5. The three tests differ only in the value of the filtration coefficient $\epsilon$ of equation (30). A constant concentration condition is applied 
at $z_{1}=0$ in all three tests.

The distributions of the relative concentration $C_{R}$ in the three tests at $t=7200 \mathrm{~s}$ are shown in Figure 11. In all tests, the SA predictions for both $N=1$ and $N=3$ are identical with the corresponding analytical solutions of Dieulin [1982].

\section{Analysis and Test Problems}

In this section the transport of various radionuclides is studied in layered systems (involving both fractured and porous layers) of different characteristics and properties. The $D_{0}$ and $\lambda$ of the radionuclides discussed here appear in Table 6 . For constant boundary conditions in any of the problems in this section, $C_{R}=1$ by definition.

\subsection{Problem 1: Importance of Fracture Misalignment}

This problem studies the importance of fracture misalignment on transport, as quantified by the concept of interlayers (discussed in Section 7.2). The following analysis focuses on the effects of the presence of such interlayers, in conjunction with other parameters of the hydrogeologic layers and of the species. The flow velocity in all cases of Problem 1 was $U=0.1 \mathrm{~m} /$ day, the system was saturated $(S=1)$, and the $z=0$ boundary was kept at a constant concentration $\left(C_{R}=1\right)$.

9.1.1. Case 1-a: Effect of fracture offset (interlayers). This case involves the transport of the nonsorbing solute species ${ }^{3} \mathrm{H}$ in a layered fractured system with fracture offsets and various interlayer characteristics. Case 1-a involves three sub-cases: 1-al, 1a2 and 1-a3. The geometry of the reference Case 1-a1 of the layered fractured system is described in Table 7, while the hydraulic properties of the fractured layers are shown in Table 8 and the sorption properties in Table 9. The three main layers (identified as Layers \# 1,3 and 5 in Table 8) were fractured media (FM), while the interlayers (identified as Layers 
\# 2 and 4) were considered to be fracture interlayers (FI, i.e., horizontal open fractures connecting the vertical fractures in the layers above and below).

The characteristics of Cases 1-a2 and 1-a3 are explained in Table 10, which shows only the differences from the base Case 1-a1. Thus, Cases 1-a2 and 1-a3 differ from Case 1-a1 in that the interlayers are porous interlayers (PI), i.e., the horizontal features connecting the fractured layers are either fractures filled with porous media or unfractured porous media. Flow and transport occurs through a porous medium with different transport behavior than in the FIs of Case 1-a1. The hydraulic properties of the porous media in the PIs in Cases $1-\mathrm{a} 2$ and $1-\mathrm{a} 3$ are the same as those of the porous matrix in the overlaying and underlying layers. In Cases 1-a2 and 1-a3, the PIs have an $X=0.025 \mathrm{~m}$ and $X=0.1 \mathrm{~m}$, respectively.

The results of the three subcases of Case 1-a are shown in Figure 12, which shows the fracture $C_{R}$. The presence of the interlayers in Figure 12 is marked by the vertical steps in the $C_{R}$ profiles (caused by the fact that Figure 12 indicates the vertical coordinate $\mathrm{z}$ and not the length of the travel path. For the nonsorbing ${ }^{3} \mathrm{H}$ and at early times, the retardation caused by the presence of the FI is measurable, as compared to the case with aligned fractures (no interlayer, denoted by NI in Figure 12-included for comparison). This was expected because of the longer travel path in the case of FIs, which increase the amount of ${ }^{3} \mathrm{H}$ diffusing into the porous matrix and result in lower fracture concentrations. At the same early times, the retardation caused by the PIs can be substantial and increases with the the half-width $b$ of the PI. These results also conform with expectations because of the slower flow velocities in the porous media of the PI (as compared to those in the fractures of the FIs), which increase the residence time and diffusion into the porous matrix.

Figure 12 also shows that the effect of the interlayers keeps decreasing with time. This was expected in Case 1-a because the travel path increase caused by the interlayers is small (as the layer half-width $X$ is only $0.25 \mathrm{~m}$ ) and ${ }^{3} \mathrm{H}$ is nonsorbing (leaving diffusion into the matrix as the only mechanism removing the radionuclide from the flowing water). 
At $t=10^{4}$ days, the presence of interlayers of any kind (FI vs. PI) has no effect on the concentration profile in the fractures.

\subsubsection{Case 1-b: Combined effect of interlayers and matrix width of the fractured}

layers. This case involves three subcases: 1-b1, 1-b2 and 1-b3 (see Table 10). Cases 1-b1, 1-b2 and 1-b3 differed from Cases $1-\mathrm{a} 1,1-\mathrm{a} 2$ and $1-\mathrm{a} 3$ in that $X=2.5 \mathrm{~m}$ instead of 0.25 $\mathrm{m}$, thus substantially increasing the travel path and residence time of ${ }^{3} \mathrm{H}$ in the interlayers. This is expected to increase retardation, especially at early times.

Figure 13 confirms this expectation. At $t=10^{2}$ days, the presence of the relatively fast flowing FI is sufficient to reduce $C_{R}$ in the fracture by about four orders of magnitude. The effect is more pronounced in Case $1-\mathrm{b} 3$ (PI with $b=0.1 \mathrm{~m}$ ). The same pattern is observed at $t=10^{3}$ days, at which time the retardation in Case $1-\mathrm{b} 3$ remains very substantial. This is caused by the reduction of the advective and dispersive components of transport (because velocity decreases as $b$ increases) in addition to the reduction of the molecular diffusion component (due to the smaller $\phi$ and $\tau$ values in the filled fracture, see equation (2)). Remarkably, stronger retardation is observed in Case 1-b1 (FI) than in case 1-b2 (PI with $b=0.025 \mathrm{~m}$ ). This is attributed to the larger solute mass in the PI, which is less affected by diffusion into the matrix (about the same in both cases). As in Case 1-a, the effect of the fracture offset (presence of interlayers) decreases with time.

The conclusion reached from these results is that the effect of fracture offsets (interlayers) increases with the matrix block size of the fractured layers. This is consistent with expectations because the travel path increases substantially in fractured system with large $X$, with a corresponding increase in residence time and diffusion into the matrix.

9.1.3. Case 1-c: Combined effect of interlayers and aperture $b$ of the fractured layers. This case involves two subcases: 1-cl and 1-c2 (see Table 10). Cases 1-c1 and 1-c2 differed from Cases $1-\mathrm{al}$ and 1 -a3, respectively, in that $b=5 \times 10^{-4} \mathrm{~m}$ instead of $5 \times 10^{-5} \mathrm{~m}$, thus substantially increasing the fracture width and, correspondingly, the mass 
of the radionuclide transported in the flowing water per unit time.

Figure 14 shows that the transport is faster in this case (compared to Cases 1-a and $1-b)$, and that the effect of fracture misalignment is minimal because the mass flow rate in this case is ten times that in Cases 1-a and 1-b. Although there may be an interlayer effect at very early times, the comparatively very large amount of available ${ }^{3} \mathrm{H}$ easily overwhelms the increased retardation capacity of the system (caused by the increased travel path, residence time, and diffusion into the matrix). The obvious conclusion is that the effect of fracture misalignment on retardation decreases with the fracture aperture of the fractured layers.

\subsubsection{Case 1-d: Combined effect of interlayers and water saturation $S$ of the} fractured layers. This case involved two subcases: 1-d1 and 1-d2 (see Table 10). Cases 1-d1 differed from Case 1-a1 in that $S^{m}=0.8$ and $S^{f}=0.5$ instead of $S^{m}=S^{f}=1$. Cases 1-d2 differed from Case $1-\mathrm{a} 3$ in that $S^{m}=S^{f}=0.8$ instead of $S^{m}=S^{f}=1$. The effect of $S$ is exhibited through its effect on the water velocity: a higher pore velocity $V$ is needed to maintain the same $U$ if $S$ decreases. Thus, faster transport was expected in this case, with a corresponding decrease in the importance of the increased travel path caused by the fracture offset.

The results in Figure 15 confirm these expectations. Transport appears faster than in Cases 1-a and 1-b, while the relative importance of the fracture offset (presence of interlayers) decreases in systems with the same water mass flow rate but with decreasing water saturation.

9.1.5. Case 1-e: Combined effect of interlayers and radionuclide properties. The transport of ${ }^{237} \mathrm{~Np}$ (a moderately sorbing radionuclide) and ${ }^{239} \mathrm{Pu}$ (a strong sorber) were studied in the fracture systems discussed in Case $1-\mathrm{a}$. The properties of ${ }^{237} \mathrm{~Np}$ and ${ }^{239} \mathrm{Pu}$ appear in Tables 6 and 9. The sorption behavior of these two species is expected to increase the retardation effect of the interlayers.

The results are shown in Figures 16 (for ${ }^{237} \mathrm{~Np}$ ) and 17 (for ${ }^{239} \mathrm{Pu}$ ), and confirm 
expectations. Despite the much longer $T_{1 / 2}$ of ${ }^{237} \mathrm{~Np}$ and ${ }^{239} \mathrm{Pu}$, transport is much slower than that for ${ }^{3} \mathrm{H}$ because of two mechanisms at work removing the solutes from the water in the fractures: diffusion and sorption. The longer travel path in the case of interlayers (fracture offsets) provides an opportunity for increased diffusion and sorption, as indicated by the comparison to the fracture $C_{R}$ profile for no interlayers. Note that sorption decreases the concentration in the liquid phase, and although the $D_{0}$ of ${ }^{3} \mathrm{H}$ is higher, diffusion is higher because the $\frac{\partial C}{\partial x}$ gradient is steeper. Retardation increases with the distribution coefficient $K_{d}$ of the radionuclide, from FIs to PIs, and with an increasing $b$ of the PIs.

\subsection{Transport of Straining ${ }^{239} \mathrm{Pu}$ Colloids in a Layered Fractured System With Fracture Misalignment}

Problem 2 describes colloid transport through the layered system with the geometry and characteristics of Case 1-a1 of Problem 1. Thus, the domain comprises three misaligned layers with the fracture offsets represented by interlayers, for a total of five layers. Colloids are subject to straining (pore-size exclusion) and filtration (a physical-chemical process of colloid attachment to active sites on the fractures and matrix).

Three cases (Cases 2-a, 2-b and 2-c) are studied in Problem 2. The properties and parameters of the base Case 2-al are listed in Table 11, and the specifics of the various cases of this problem are listed in Table 12. Two colloids are compared in all the studies: a $5 \mathrm{~nm}$ colloid and a $500 \mathrm{~nm}$ colloid. Flow velocities, water saturations and boundary conditions were as in Problem 1.

9.2.1. Case 2-a: No matrix deposition. In this case, colloids were deposited in the fractures, but there was no colloid filtration in the matrix. The FRACL results in Cases 2-al and 2-a2 are shown in Figures 18 and 19, which also include the $C_{R}$ profile for aligned fractures (no interface, NI) for reference. The following observations are made:

1. At the straining interface (i.e., at the boundary of a fracture with a PI) $C_{R}$ exhibits a 
localsteeppeak, and $C_{R}$ caneasilyexceedthevalueof 1 . This indicatesaconcentration that exceeds the source concentration and is an expected consequence of straining.

2. Because of their larger size and enhanced pore size exclusion, the fracture $C_{R}$ of larger colloids is significantly higher than that for smaller colloids if no PIs are involved.

3. Figures 18 and 19 confirm the expectation that PIs can have a very strong retardation effect on transport because they are far more effective strainers than FIs (in which straining may be provided by a narrowing of the fracture). Consequently, the fracture $C_{R}$ of larger colloids after a PI is significantly lower than that of smaller colloids. This indicates that filled fractures or flow through the matrix between fractures can drastically reduce colloid concentrations in the fractures.

4. A very important observation is that larger colloids exhibit faster transport in the fractures than smaller ones. This is because of their size, which affects three processes. Larger colloids move faster $\left(1<f_{v} \leq 1.5\right.$ because they are channeled toward the middle of the fractures and pores where the flow velocity is maximum. As can be seen from equation (27), the coefficient of colloidal diffusion decreases in larger colloids, thus limiting diffusion intothe matrix and leaving larger colloidal loads in the fractures. Penetration into the matrix (and, consequently, removal from the fractures) is further restricted by pore size exclusion.

5. The ${ }^{239} \mathrm{Pu}$ colloid front moves at least one to two orders of magnitude faster than the front of the solute ${ }^{239} \mathrm{Pu}$ (a strongly-sorbing species). This is true even when colloids are transported through straining (and strongly retarding) PIs, and appears to confirm previous laboratory and field studies [McCarthy and Zachara, 1989; Buddemeier and Hunt, 1988, Kersting et al., 1999]. The obvious implication is that, if the geochemical conditions favor the creation and stability of radioactive colloids, these can move much faster through the geologic system than the corresponding solutes of strongly sorbing species, because colloid filtration is not as important a retardation mechanism as solute 
sorption.

6. At longer times, the fracture $C_{R}$ profiles for both the $5 \mathrm{~nm}$ colloid and the $500 \mathrm{~nm}$ colloid are about the same if no PIs are involved.

7. The fracture concentrations $C_{R}$ continue to grow for a long time behind the straining surface, and eventually reach steady-state levels that, for larger colloids, can be hundreds of times higher than the source concentrations. In this example, the 500 $\mathrm{nm}$ colloid before the first PI reaches a $C_{R}$ of about 300 at $t=10^{4}$ days; at the same time and location, the $5 \mathrm{~nm}$ colloid reaches a $C_{R} \equiv 20$.

Figure 20 provides a more detailed view of the steep $C_{R}$ peaks behind the PIs (i.e., the straining interfaces) and their evolution over time. Note that the $500 \mathrm{~nm}$ profile reaches a steady state far earlier than the smaller colloid.

9.2.2. Case 2-b: Colloid filtration in the matrix. The specifics of the various subcases of Case 2-b are listed in Table 12. The only difference in geometry is the wider matrix block of the fractured system under study. The results at $t=10^{4}$ days are shown in Figure 21, which also includes the solution for aligned fractures (denoted by NI, i.e., no interlayer).

The general pattern of fracture $C_{R}$ profile here is similar to that in Figures 18 and 19 , and is characterized by steep concentration spikes behind straining PI interfaces. There are, however, some significant differences.

One of the most remarkable differences is that the effect of matrix filtration is far more pronounced in the case of the $5 \mathrm{~nm}$ colloid than in the larger $500 \mathrm{~nm}$ colloid. Thus, the presence of matrix filtration leads to significantly lower concentrations than the analogous results without filtration, and is consistent with the diffusion of relatively large amounts of the smaller colloids into the matrix. This holds true for any of the subcases (2-b1 through 2-b4), as well as for the reference case of aligned fractures. In subcase 2-b3 (which assumes transport through a PI with $b=0.1 \mathrm{~m}-\mathrm{PI}(\mathrm{b})$ in Figure 21 ), the colloids breach the first 
PI, but the combined straining-filtration effect at the interface of the fractured layer and the second PI is so strong that the $5 \mathrm{~nm}$ colloids have not yet entered the fractures in the underlying layer after $t=10^{4}$ days.

In subcases 2-b2 (thinner porous flowing section - PI(a) in Figure 21) and 2-b4 (thinner flowing section and weaker filtration - PI(c) in Figure 21), the colloid manages to breach the PIs, but the retardation (over the no-filtration cases of Figure 19) is very significant. This is important, given the fact that these very large retardations are caused by an additional travel path of only $0.25 \mathrm{~m}$ in each interlayer. Note that, as expected, the weaker matrix filtration in subcase 2-b4 leads to higher $C_{R}$ in the fractures. The fracture $C_{R}$ profiles for the cases of NI and FI practically coincide.

An interesting observation is that the $C_{R}$ profiles for NI and FI of the $500 \mathrm{~nm}$ colloid show little difference from those without matrix filtration in Figure 19. This indicates that matrix deposition does not significantly affect the transport of this colloid for NI or FI, and is attributed to the fact that so little of it enters the matrix because of straining. The effect of straining at the interface with PIs, however, has significant effects on the fracture $C_{R}$, which are now much lower than the corresponding profiles in Figure 19. As in the case of the $5 \mathrm{~nm}$ colloid, no $500 \mathrm{~nm}$ colloids appear in the fractures of the underlying layer when the PI has a $b=0.1 \mathrm{~m}$ (Case 2-b3 - PI(c) in Figure 21). In Cases 2-b2 and 2-b4, the $C_{R}$ concentrations are (a) lower than the corresponding ones in Figure 19 (because of colloid removal by the porous medium in the PI), but (b) higher than the ones for the $5 \mathrm{~nm}$ colloids in Figure 21 (because far fewer $500 \mathrm{~nm}$ colloids than $5 \mathrm{~nm}$ colloids enter the matrix).

\subsubsection{Case 2-c: Colloid filtration in fractured media with wide matrix blocks.} The specifics of the various subcases of Case 2-c are listed in Table 12. The results at $t=10^{4}$ days are shown in Figure 22, which also includes the solution for aligned fractures (denoted by NI).

Figure 22 shows the same general pattern of colloid transport observed in Figures 18 
through 21 and a prominent colloid accumulation behind straining PI interfaces. For no matrix deposition (denoted by NMD in Figure 22), there is practically no difference in the fracture $C_{R}$ profiles between Case 2-c and 2-a (see Figure 20) for both the $5 \mathrm{~nm}$ and the $500 \mathrm{~nm}$ colloids.

Thus, the effect of a larger matrix block (and, consequently, longer travel path) is practically negligible at $t=10^{4}$ days when matrix deposition is not considered. The same holds true for the profile with NI and matrix deposition (denoted by WMD in Figure 22) for both colloids, and the profile for FI and the larger colloid (because of the limited diffusion and the pore size exclusion). The $5 \mathrm{~nm}$ fracture $C_{R}$ profile for matrix filtration does show a small difference from the one for FI, which is attributed to the increased filtration and diffusion into the matrix due to the longer travel path of the colloid in the FI in Case 2-c (which is 10 times longer than the reference travel path in the FI in Case 2-a).

The most pronounced effect is noticed in the case of a PI, which strains colloids and provides more opportunities for filtration in the matrix of the interlayer. In both colloids, the longer travel path in the PI (over the reference in Case 2-a) results in profiles that indicate the colloids cannot even breach the first PI after $t=10^{4}$ years. Thus, the fracture misalignment and the transport through a longer porous pathway (a consequence of the larger matrix blocks) prevent the migration of the colloids in the fracture system.

\subsection{Problem 3: Radioactive Solute Transport in a Complex Multi-Layered System}

The complex geological system in Problem 3 is comprised of 14 layers and interlayers of fractured and porous media. The geometry and configuration of the system are described in Table 13, and the rock properties and conditions are listed in Table 14. Linear equilibrium sorptionis assumed, and thesorption coefficientsof thevariousradionuclides inthefractures and in the matrix of the various layers $\left(K_{d}^{f}\right.$ and $K_{d}^{m}$, respectively) are listed in Table 15. 
The water velocity $U$ at $z=0$ is as in Problem 1 .

9.3.1. ${ }^{3} \mathrm{H}$ Transport. The fracture $C_{R}$ profiles of the nonsorbing ${ }^{3} \mathrm{H}$ for both constant concentration (CC) and decaying (radioactively) concentration (DC) at the $z=0$ boundary are shown in Figure 23, which includes observations at the following times: $t_{1}=10^{4}$ days, $t_{2}=5 \times 10^{4}$ days, $t_{3}=10^{5}$ days, $t_{4}=2.5 \times 10^{5}$ days and $t_{5}=5 \times 10^{5}$ days.

The various layers can be generally identified by a change in the $C_{R}$ slope, while the interlayers are indicated by vertical sections of the $C_{R}$ curves (as the abscissa is the $z$ coordinate rather than the travel path). For a $\mathrm{CC}$ boundary, the $C_{R}$ distribution reaches a steady state for $t \geq t_{4}$. As expected, the effect of the DC boundary is a $C_{R}$ profile that is progressively lower than the one for a $\mathrm{CC}$ boundary, never reaches steady state, and is outside the $C_{R}$ range $\left(<10^{-9}\right)$ for $t \geq t_{4}$. This example demonstrates that a complex problem of transport in a multilayered system can be easily handled by FRACL, which yields semianalytical (SA) solutions in less than $10 \mathrm{~s}$.

9.3.2. ${ }^{99} \mathrm{Tc}$ Transport. ${ }^{99} \mathrm{Tc}$ (in its pertechnate $\mathrm{TcO}_{4}^{-}$speciation) is a non-sorbing radionuclide with a longer half life than ${ }^{3} \mathrm{H}$ (see Table 6). Two boundary conditions were considered in this case: a CC boundary and a piece-wise continuous (step) concentration (PC) boundary, i.e.,

$$
C_{R}(z=0)= \begin{cases}1 & \text { for } t \leq 5 \times 10^{4} \text { days } \\ 0 & \text { for } t>5 \times 10^{4} \text { days }\end{cases}
$$

The $C_{R}$ profiles in the fractures of the layered geologic system (at the same times as in the case of ${ }^{3} \mathrm{H}$ in Section 9.3.1) are shown in Figure 24. The effect of the longer half life is evident in the $C_{R}$ profile for CC boundary, which indicates that ${ }^{99} \mathrm{Tc}$ advances much further in the formation than ${ }^{3} \mathrm{H}$ at the same times (the difference is due to radioactive decay), and does not appear to have reached steady state at $t=t_{5}$. The change in the boundary concentration over time in the $\mathrm{PC}$ boundary case results in $C_{R}$ profiles that show aprogressivelylarger(withtime) ${ }^{99} \mathrm{Tc}$-freezoneneartheboundary, whilethe $C_{R}$ further into 
the formation keeps decreasing and deviating from that for constant boundary concentration (with which it coincides fully or in part for $t \leq t_{4}$ ).

As indicated in the case of ${ }^{3} \mathrm{H}$, the various layers and interlayers can be generally identified from changes in the $C_{R}$ slope. Transport in fast flowing fractures (e.g., in the case of narrow fractures with large matrix blocks under a layer of wider fractures and narrow matrix blocks) can also be identified by a near-horizontal portion of the $C_{R}$ profile. This example (which takes less than $15 \mathrm{sec}$ to run) confirms the ability of FRACL to solve the problem of transport of a decaying radionuclide in a complex geological system.

9.3.3. ${ }^{237} \mathrm{~Np}$ Transport. The fracture $C_{R}$ profile of the moderately sorbing ${ }^{237} \mathrm{~Np}$ for a CC boundary is shown inFigure 25 . Note that the observation times here are: $t_{1}=5 \times 10^{4}$ days, $t_{2}=10^{5}$ days, $t_{3}=5 \times 10^{5}$ days, $t_{4}=10^{6}$ days, $t_{5}=2.5 \times 10^{6}$ daysand $t_{6}=5 \times 10^{6}$ days.

The slower transport of ${ }^{237} \mathrm{~Np}$ (compared to that of ${ }^{99} \mathrm{Tc}$ ) is caused by sorption and, to a far lesser extent, by increased diffusion into the matrix. Despite its longer half-life, the transport of ${ }^{237} \mathrm{~Np}$ appears to be about an order of magnitude slower than that of ${ }^{99} \mathrm{Tc}$, and does not appear to have reached steady state at $t=t_{6}$.

The $C_{R}$ profiles along the $x$ axis in the matrices of the various layers at $t=t_{6}$ are shown in Figure 26. The different shape of the curves is a function of their location (with respect to the $z=0$ boundary and to the solute front) and of the sorption properties of the matrix in the various layers.

\subsection{Problem 4: Solute Transport of a Three-Member Radioactive Decay Chain in a Complex Multi-Layered System}

Problem 4 describes the transport of the radioactive chain

$$
{ }^{239} \mathrm{Pu} \rightarrow{ }^{23.5} \mathrm{U} \rightarrow{ }^{2.31} \mathrm{~Pa}
$$

through the complex multilayered system described in Problem 3 (Tables 12 and 13). The 
sorption coefficients $K_{d}^{f}$ and $K_{d}^{m}$ of the ${ }^{239} \mathrm{Pu}$ parent in the various layers are listed in Table 15. The sorption coefficients of ${ }^{235} \mathrm{U}$ and ${ }^{231} \mathrm{~Pa}$ in the fractures and in the matrix were assumed to be $5 \%$ and $50 \%$ of the ${ }^{239} \mathrm{Pu}$ ones, respectively. $C_{R}$ profiles of the three radionuclides were obtained at the following observation times: $t_{1}=10^{5}$ days, $t_{2}=10^{6}$ days, $t_{3}=10^{7}$ days, $t_{4}=10^{8}$ days, $t_{5}=10^{9}$ days, and $t_{6}=10^{10}$ days. Two boundary conditions were considered: a CC and a DC boundary.

9.4.1. ${ }^{239} \mathrm{Pu}$ Transport. Figure 27 shows the $C_{R}$ profiles of ${ }^{239} \mathrm{Pu}$ in the fractures for constant boundary concentration and a decaying boundary concentration. There is no or little deviation of the two curves until $t=t_{3}$. The fracture $C_{R}$ in DC case at $t=t_{4}$ is substantially lower than that of the $\mathrm{CC}$ case, and the $C_{R}$ for a DC boundary is less than $10^{-9}$ for $t \geq t_{5}$

An interesting observation is that, for a $\mathrm{CC}$ boundary, the ${ }^{239} \mathrm{Pu}$ front does not advance deep into the formation despite observation times orders of magnitude larger than those for the ${ }^{237} \mathrm{~Np}$ transport. This is due to the very strong sorption of ${ }^{239} \mathrm{Pu}$ onto the matrix and fractures of the layers and, to a lesser extent, the shorter half life of ${ }^{239} \mathrm{Pu}$ (compared to that of ${ }^{237} \mathrm{~Np}$. Note that the $C_{R}$ profile appears to have reached steady state at $t \geq t_{5}$.

In addition to the transport of the members of the chain, the transport of ${ }^{239} \mathrm{Pu}$ was studied separately, assuming a CC boundary and a $r \leq 1$ (see Equation (38) and the corresponding discussion). This describes a situation in which not all the contact area between fracture and matrix contributes to transport (e.g., because of a partially dry fracture which constitutes a discontinuity in the water phase). In this case, $r=S_{w}^{f}$ in the fractured layers and interlayers (FM or FI), and $r=1$ elsewhere.

The effect of $r \leq 1$ in Figure 28 appears to have a substantial effect on transport, and results in a ${ }^{239} \mathrm{Pu}$ front that reaches much further (i.e., about three time deeper) in the geologic profile than that for $r=1$. This is a direct consequence of a reduced area for ${ }^{239} \mathrm{Pu}$ diffusion from the fractures into the matrix, which leaves a larger amount of ${ }^{239} \mathrm{Pu}$ in 
the fractures where advection is fast and sorption relatively small (compared to the matrix). Thus, the transport of strongly sorbing radionuclides in fractured systems may be strongly influenced (enhanced) by partially dry fractures.

9.4.2. ${ }^{235} \mathrm{U}$ Transport. The fracture $C_{R}$ profiles of ${ }^{235} \mathrm{U}$ for $\mathrm{CC}$ and $\mathrm{DC}$ boundaries and for $t \leq t_{4}$ are shown in Figure 29. The $C_{R}$ of the DC solution always exceeding that from the CC solution, and significantly so (as imposed) in the vicinity of $z=0$. A very significant observation is that, in either case, $C_{R} \simeq 1$ for $t \geq t_{4}$ in the top $120 \mathrm{~m}$ of the domain. This is even more the case in Figure 30, which shows the $C_{R}$ of ${ }^{235} \mathrm{U}$ for $t \geq 4$ and gives a more detailed picture of the $C_{R}$ distribution near the value of 1 . The results in Figures 29 and 30, in conjunction with the observations from Figure 27, indicate that for $t \geq t_{4}$, practically all of the radionuclide that advances deep into the formation is the ${ }^{235} \mathrm{U}$ daughter. The transport of ${ }^{235} \mathrm{U}$ is faster, the front reaches deeper, and $C_{R} \simeq 1$ because ${ }^{235} \mathrm{U}$ is generally weaker sorbing than ${ }^{239} \mathrm{Pu}$ and it has an extremely long half life. The obvious implication is that studies of ${ }^{239} \mathrm{Pu}$ transport cannot neglect the transport of the ${ }^{235} \mathrm{U}$ daughter, which is the dominant radionuclide at longer times.

Note from Figure 30 that, for $t=t_{4}$ and a DC boundary, $C_{R}>1$, i.e., the ${ }^{235} \mathrm{U}$ concentration in the fractures exceeds the initial ${ }^{239} \mathrm{Pu}$ concentration at the $z=0$ boundary. This is possible because the boundary (which introduces a radionuclide mixture composed of all the members of the decay of the chain as ${ }^{239} \mathrm{Pu}$ decays) is now contributing a stream of almost $100 \%{ }^{235} \mathrm{U}$, which is added to the ${ }^{235} \mathrm{U}$ produced from the (almost complete) decay of ${ }^{239} \mathrm{Pu}$ already in the fractures and matrix of the system.

As expected, the $C_{R}$ from the CC solution at $t=t_{4}$ is lower than that from the DC solution (Figure 29). For $t>t_{4}$, the CC solutions exceed the DC solutions because the decay of the ${ }^{235} \mathrm{U}$ at the boundary is beginning to have an effect on the fracture distribution of $C_{R}$. This is particularly evident at $t=t_{6}$. Note that steady state is not reached (in either the $\mathrm{CC}$ or the $\mathrm{DC}$ boundary cases) even after $t_{6}=10^{10}$ days because of the extremely long 
half-life of ${ }^{235} \mathrm{U}$.

9.4.3. ${ }^{231} \mathrm{~Pa}$ Transport. The fracture $C_{R}$ profiles of ${ }^{231} \mathrm{~Pa}$ for $\mathrm{CC}$ and $\mathrm{DC}$ boundaries are shown in Figure 31. The $C_{R}$ levels of ${ }^{231} \mathrm{~Pa}$ are quite low because of the very long half life of its ${ }^{235} \mathrm{U}$ parent, its own shorter half life, and its stronger tendency to sorb. The $C_{R}$ increases with time for both DC and CC boundaries. The CC profile has always lower concentrations because there are all derived solely from the decay of ${ }^{235} \mathrm{U}$ (the boundary doesnotsupplyanyadditional ${ }^{231}$ PainaCCregime). Note thatineither case,concentrations reach a steady state at about $t=t_{4}$.

These examples demonstrate that the FRACL code (i.e., the FORTRAN implementation of the semianalytical solutions developed in Sections 2.0 through 7.0 ) is capable of handlingthetransport ofallthemembersof decaychains incomplexmultilayeredgeological systems and under a variety of boundary conditions.

\subsection{Problem 5: Transport of a Radioactive Colloid Parent and a Solute Daughter in a Complex Multi-Layered System}

Problem 5 describes the transport of the radioactive chain ${ }^{239} \mathrm{Pu} \rightarrow{ }^{235} \mathrm{U}$, in which ${ }^{239} \mathrm{Pu}$ is a $10 \mathrm{~nm}$ true colloid (with the properties of $\mathrm{PuO}_{2}$ ), and ${ }^{235} \mathrm{U}$ is a solute. The radionuclides are transported through the complex layered system of Problems 3 and 4, and $f_{v}=1$ in all the layers. The colloid filtration is described by the linear kinetic model of equation (29). The filtration and straining parameters in the matrix and in the fractures are listed in Table 16, and the sorption coefficients of the ${ }^{235} \mathrm{U}$ daughter are as in Problem 4. $C_{R}$ profiles of the three radionuclides are obtained at the following observation times: $t_{1}=10^{5}$ days, $t_{2}=10^{6}$ days, and $t_{3}=10^{7}$ days (sufficiently short so that the colloidal particle diameter remains practically unchanged). A CC boundary condition is assumed.

9.5.1. Transport of the ${ }^{239} \mathrm{Pu}$ colloid. Figure 32 shows the $C_{R}$ profiles of the ${ }^{239} \mathrm{Pu}$ in the fractures at $t_{1}, t_{2}$ and $t_{3}$. The $C_{R}$ of the solute ${ }^{239} \mathrm{Pu}$ (Figure 27) at the same times 
are included for reference. The colloid $C_{R}$ profiles are typical of straining colloids (e.g., Figures 18 to 22), and exhibit concentration spikes that can exceed the input concentrations immediately behind straining interfaces (porous media layers or filled fractures). A very important observation is that the colloid front advances about six times deeper into the formation than the corresponding ${ }^{239} \mathrm{Pu}$ solute. This is because matrix diffusion is far less important in the case of the colloid because of its larger size, which results in larger colloid concentrations in the fractures where transport is fast. Additionally, the strong ${ }^{239} \mathrm{Pu}$ sorption is a much more efficient retardation mechanism than the colloid filtration.

9.5.2. Transport of the solute ${ }^{235} \mathrm{U}$ daughter of the colloid. The fracture $C_{R}$ profiles of the solute ${ }^{235} \mathrm{U}$ daughter of ${ }^{239} \mathrm{Pu}$ colloid is shown in Figure 33, which also includes the $C_{R}$ profiles of the ${ }^{235} \mathrm{U}$ daughter of ${ }^{239} \mathrm{Pu}$ solute (Figure 29). The faster transport of the colloid parent is reflected in the solute daughter $C_{R}$ profiles, which show that the ${ }^{235} \mathrm{U}$ moves faster and advances deeper in the formation than the daughter of the ${ }^{239} \mathrm{Pu}$ solute.

Theconcentration spikesoftheparent(causedbycolloidaccumulationbehind straining interfaces) are evident in the $C_{R}$ of the ${ }^{235} \mathrm{U}$ daughter at $t_{1}$, but these are progressively attenuated (but discernible) as time advances. Note that the ${ }^{235} \mathrm{U}$ concentration near the $z_{1}=0$ boundary is lower than in the case of ${ }^{235} \mathrm{U}$ from the a solute ${ }^{239} \mathrm{Pu}$ parent. This is caused to the limited (compared to sorption) filtration of the ${ }^{239} \mathrm{Pu}$ colloids in the fractures and the matrix in the vicinity of the boundary, which in turns limits the amount of parent available for ${ }^{235} \mathrm{U}$ generation through decay.

\section{Summary}

In this paper, semianalytical solutions are developed for the problem of transport of radioactiveorreactivetracers(solutesorcolloids)throughalayeredsystemofheterogeneous fracturedmediawith misalignedfractures. Thesolutionsallowanynumberand combination 
of fractured and/or porous layers that can vary in hydraulic and transport properties, fracture frequency, water saturation, fracture flow, and fracture-matrix interaction.

The tracer transport equations in the matrix account for (a) diffusion, (b) solute surface diffusion, (c) mass transfer between the mobile and immobile water fractions, (d) linear kinetic or equilibrium physical, chemical or combined solute sorption or colloid filtration, and (e) radioactive decay or first order chemical reactions. Any number of radioactive decay daughter products (or products of a linear, first-order reaction chain) can be tracked. The tracer transport equations in the fractures account for the same processes, as well as for advection and hydrodynamic dispersion. Additionally, the colloid transport equations account for straining and velocity adjustments related to colloidal size. A wide array of boundary conditions (constant or time-variable, concentration or flux) can be accommodated.

Analytical solutions describing transport in the fracture and the matrix of each layer are first obtained in the Laplace space. These are impossible to invert analytically, and are numerically inverted by the method of DeHoog et al. [1982] to yield the solutions in time.

The semianalytical solutions are verified against analytical solutions of limiting cases of solute and colloid transport in a fractured medium. Additional verification is provided by comparisons against analytical solutions of transport in porous (unfractured) media.

The semianalytical solutions are then tested in a series of hypothetical problems of increasing complexity. The effect of important parameters on the transport of ${ }^{3} \mathrm{H},{ }^{237} \mathrm{~Np}$ and ${ }^{239} \mathrm{Pu}$ (and its daughters) is investigated in several test problems involving layered heterogeneous geological systems. Fracture misalignment appears to significantly affect transport if water flow (and, consequently, transport) between the fractures of the overlaying and the underlying layers occurs through a porous connecting pathway. Test problems involving radioactive $\left({ }^{239} \mathrm{Pu}\right)$ colloid transport in multilayered systems indicate significant colloid accumulations at straining interfaces but much faster transport of the colloid than 
the corresponding strongly sorbing solute species. Solute daughters of colloid parents are affected by the faster transport of the parents, and exhibit faster transport than the same daughters from solute parents.

The semianalytical solutions are very computationally efficient, requiring less than 10 seconds of execution time for the examples studied in this paper. The results of the test problems indicate that the semianalytical solutions can easily solve the problem of transport of parent and daughter radioactive species in multilayered heterogeneous systems under a variety of boundary conditions.

Acknowledgments. ThisworkwassupportedbytheDirector,OfficeofCivilianRadioactive Waste Management, U.S. Department of Energy, through Memorandum Purchase Order EA9013MC5X between Bechtel SAIC Company, LLC and the Ernest Orlando Lawrence Berkeley National Laboratory (Berkeley Lab). The support is provided to Berkeley Lab through the U.S. Department of Energy Contract No. DE-AC03-76SF00098. Drs. Chao Shan and John Apps are thanked for their insightful review comments.

\section{Appendix A: The $H_{\nu}$ and $T_{\nu, \kappa}$ Coefficients}

For Case $2(X \rightarrow \infty)$, the $H_{\nu} \equiv H_{\nu}^{e}$ of the first five members of a radioactive or reactive chain $(\nu=1, \ldots, 5)$ are

$$
\begin{aligned}
H_{1}^{e}= & \widehat{C}_{1}^{f} \\
H_{2}^{e}= & \widehat{C}_{2}^{f}-A_{21} \widehat{C}_{1}^{f} \\
H_{3}^{e}= & \widehat{C}_{3}^{f}-A_{32} \widehat{C}_{2}^{f}+A_{21}\left(A_{32}-A_{31}\right) \widehat{C}_{1}^{f} \\
H_{4}^{e}= & \widehat{C}_{4}^{f}-A_{43} \widehat{C}_{3}^{f}+A_{32}\left(A_{43}-A_{42}\right) \widehat{C}_{2}^{f} \\
& \quad-A_{21}\left[A_{32}\left(A_{43}-A_{42}\right)-A_{31}\left(A_{43}-A_{41}\right)\right] \widehat{C}_{1}^{f}
\end{aligned}
$$




$$
\begin{aligned}
& H_{5}^{e}=\widehat{C}_{5}^{f}-A_{54} \widehat{C}_{4}^{f}+A_{43}\left(A_{54}-A_{53}\right) \widehat{C}_{3}^{f} \\
& -A_{32}\left[A_{43}\left(A_{54}-A_{53}\right)-A_{42}\left(A_{54}-A_{52}\right)\right] \widehat{C}_{2}^{f} \\
& +A_{21}\left\{A_{32}\left[A_{43}\left(A_{54}-A_{53}\right)-A_{42}\left(A_{54}-A_{52}\right)\right]\right. \\
& \left.\quad-A_{31}\left[A_{43}\left(A_{54}-A_{53}\right)-A_{41}\left(A_{54}-A_{51}\right)\right]\right\} \widehat{C}_{1}^{f}
\end{aligned}
$$

in which the $m$ superscript of the $A$ factors (equation (52)) are omitted for simplicity. The terms $T_{\nu, \kappa}$ inequation (53)can beeasilyidentified byinspection. Byfollowing theemerging pattern, the development of the expressions for $H_{\nu}$ for $\nu>5$ is tedious but straightforward.

The $H_{\nu} \equiv H_{\nu}^{c}$ expressions (corresponding to Case 1) are entirely analogous, and are derived by dividing $H_{\nu}^{e}$ by $\cosh \left(\theta_{\nu} X\right)$. For example, for $\nu=2$,

$$
H_{2}^{c}=\frac{\widehat{C}_{2}^{f}}{\cosh \left(\theta_{2} X\right)}-\frac{A_{21} \widehat{C}_{1}^{f}}{\cosh \left(\theta_{2} X\right)}
$$

\section{Appendix B: The $W_{\nu}$ and $\gamma_{\nu, \kappa}$ Coefficients}

For Case $2(X \rightarrow \infty)$, the $W_{\nu} \equiv W_{\nu}^{e}$ of the first 5 members of a radioactive or reactive chain $(\nu=1, \ldots, 5)$ are

$$
\begin{aligned}
& W_{1}^{e}=\theta_{1} C_{1}^{f} \\
& W_{2}^{e}=\theta_{2} C_{2}^{f}+A_{21}\left(\theta_{1}-\theta_{2}\right) \widehat{C}_{1}^{f} \\
& W_{3}^{e}=\theta_{3} C_{3}^{f}+A_{32}\left(\theta_{2}-\theta_{3}\right) \widehat{C}_{2}^{f}+A_{21}\left[A_{31} \theta_{1}-A_{32} \theta_{2}+\left(A_{32}-A_{31}\right) \theta_{3}\right] \widehat{C}_{1}^{f} \\
& W_{4}^{e}=\theta_{4} C_{4}^{f}+A_{43}\left(\theta_{3}-\theta_{4}\right) \widehat{C}_{3}^{f}+A_{32}\left[A_{42} \theta_{2}-A_{43} \theta_{3}+\left(A_{43}-A_{42}\right) \theta_{4}\right] \widehat{C}_{2}^{f} \\
& \quad+A_{21}\left\{A_{31} A_{41} \theta_{1}-A_{32} A_{42} \theta_{2}+A_{43}\left(A_{32}-A_{31}\right) \theta_{3}\right. \\
& \left.\quad-\left[A_{32}\left(A_{43}-A_{42}\right)-A_{31}\left(A_{43}-A_{41}\right)\right] \theta_{4}\right\} \widehat{C}_{1}^{f}
\end{aligned}
$$




$$
\begin{gathered}
W_{5}^{e}=\theta_{5} C_{5}^{f}+A_{54}\left(\theta_{4}-\theta_{5}\right) \widehat{C}_{4}^{f}+A_{43}\left[A_{53} \theta_{3}-A_{54} \theta_{4}+\left(A_{54}-A_{53}\right) \theta_{5}\right] \widehat{C}_{3}^{f} \\
+A_{32}\left\{A_{42} A_{52} \theta_{2}-A_{43} A_{53} \theta_{3}+A_{54}\left(A_{43}-A_{42}\right) \theta_{4}\right. \\
\left.\quad-\left[A_{43}\left(A_{54}-A_{53}\right)-A_{42}\left(A_{54}-A_{52}\right)\right] \theta_{5}\right\} \widehat{C}_{2}^{f} \\
+A_{21}\left\{A_{31} A_{41} A_{51} \theta_{1}-A_{32} A_{42} A_{52} \theta_{2}+A_{43} A_{53}\left(A_{32}-A_{31}\right) \theta_{3}\right. \\
-A_{54}\left[A_{32}\left(A_{43}-A_{42}\right)-A_{31}\left(A_{43}-A_{41}\right)\right] \theta_{4} \\
+\left[A_{32}\left[A_{43}\left(A_{54}-A_{53}\right)-A_{42}\left(A_{54}-A_{52}\right)\right]\right. \\
\left.\left.-A_{31}\left[A_{43}\left(A_{54}-A_{53}\right)-A_{41}\left(A_{54}-A_{51}\right)\right]\right] \theta_{5}\right\} \widehat{C}_{1}^{f}
\end{gathered}
$$

in which the $m$ superscript of the $A$ factors (equation (52)) are omitted for simplicity.

We obtain $W_{\nu}^{c}$ for Case 1 by replacing $\theta_{\nu}$ by $\theta_{\nu} \tanh \left(\theta_{\nu} X\right)$ in $W_{\nu}^{e}$. Thus, for $\nu=2$ and Case 1,

$$
W_{2}^{c}=\theta_{2} \tanh \left(\theta_{2} X\right) C_{2}^{f}+A_{21}\left[\theta_{1} \tanh \left(\theta_{1} X\right)-\theta_{2} \tanh \left(\theta_{2} X\right)\right] \widehat{C}_{1}^{f}
$$

The terms $\gamma_{\nu, \kappa}$ are easy to obtain from (59) and the $W_{\nu}^{c}, W_{\nu}^{e}$ expressions by inspection. Extension for $\nu>5$ follows the same pattern.

\section{Appendix C: The $B_{\nu, \kappa}^{ \pm}$Coefficients}

The $B_{\nu, \kappa}^{ \pm}$coefficients of up to the first 5 members of a radioactive or reactive chain $(\nu=1, \ldots, 5, \kappa=1, \ldots, \nu-1)$ are given by the following general expressions:

$$
\begin{gathered}
B_{\nu, \nu-1}^{ \pm}=\gamma_{\nu, \nu-1} f^{q}-G_{\nu}^{f} \\
B_{\nu, \nu-2}^{ \pm}=\left(\gamma_{\nu, \nu-1} f^{q}-G_{\nu}^{f}\right) A_{\nu-1, \nu-2}^{ \pm}+\gamma_{\nu, \nu-2} f^{q} \\
B_{\nu, \nu-3}^{ \pm}=\left(\gamma_{\nu, \nu-1} f^{q}-G_{\nu}^{f}\right) A_{\nu-1, \nu-3}^{ \pm}+\left(\gamma_{\nu, \nu-2} A_{\nu-2, \nu-3}^{ \pm}+\gamma_{\nu, \nu-3}\right) f^{q} \\
B_{\nu, \nu-4}^{ \pm}=\left(\gamma_{\nu, \nu-1} f^{q}-G_{\nu}^{f}\right) A_{\nu-1, \nu-4}^{ \pm}+\left(\gamma_{\nu, \nu-2} A_{\nu-2, \nu-4}^{ \pm}+\gamma_{\nu, \nu-3} A_{\nu-3, \nu, 4}^{ \pm}+\gamma_{\nu, \nu-4}\right) f^{q}
\end{gathered}
$$


The coefficients $A^{ \pm}$needed for the computation of $B^{ \pm}$are obtained from equation (63). All other terms are as discussed in Section 5.2. Extension for $\nu>5$ follows the same pattern.

\section{References}

Abdel-Salam, A., and C.V. Chrysikopoulos, Modelling colloid transport and deposition in saturated fractures, Colloids and Surfaces A, 121, 189-202, 1997.

Abdel-Salam, A., and C.V. Chrysikopoulos, Analysis of a model of contaminant transport in fractured media in the presence of colloids, J. Hydrol., 165, 261-281, 1995.

Abdel-Salam, A., and C.V. Chrysikopoulos, Analytical solutions for one-dimensional colloid transport in saturated fractures, Adv. Water Resour., 17, 283-296, 1994.

Bandurraga, T.M., and G.S. Bodvarsson, Calibrating hydrogeologic parameters for the 3D site-scale unsaturated zone model of Yucca Mountain, Nevada, J. Contam. Hydrol., $38(1-3), 25-46,1999$.

Bear, J., Hydraulics of Groundwater, McGraw-Hill, New York, 1979.

Berry, J. A., and K. A. Bond, Studies of the extent of surface diffusion in the migration of radionuclides through geological media, Radiochimica Acta, 58/59, 329-335, 1992.

Bird, R.B., W.E.Stewart, and N.E.Lightfoot, Transport Phenomena, John Wiley, New York, 1960.

Bowen, D.D., and N. Epstein, Fine particle deposition in smooth parallel plate channels, $J$. Colloid Interface Sci., 72, 81-97, 1979.

Buddemeier, R.W., and J.R. Hunt, Transport of colloidal contaminants in groundwater: Radionuclide migration at the Nevada Test Site, Appl. Geochem., 3, 535-548, 1988.

Cameron, D.R., and A. Klute, Convective-dispersive solute transport with a combined equilibrium and kinetic adsorption model, Water Resour. Res., I3(1), 183-188, 1977.

Cho, C.M., Convective transport of ammonium with nitrification in soil, Can. J. Soil Sci., $51,339-350,1971$. 
Çorapçioglu, M.Y., N.M. Abboud, and A. Haridas, Governing equations for particle transport in porous media, in Advances in Transport Phenomena in Porous Media, edited by : J. Bear and M.Y. Çorapçioglu, Series E: Applied Sciences Series No. 128, Martinus Nijhoff, Dordrecht, The Netherlands, 1987.

Cook, A. J., A desk study of surface diffusion and mass transport in clay, Report WE/88/34, Commission of the European Communities, Directorate-General, Sciences Research and Development, Louxemburg, 1989.

DeHoog, F.R., J.H. Knight, and A.N. Stokes, An improved method for numerical inversion of Laplace transforms, SIAM J. Sci. Stat. Comput., 3(3), 357-366, 1982.

de Marsily, G., Quantitative Hydrogeology, Academic Press, San Diego, 1986.

Dieulin, J., Filtration de colloides d' actinides par une colonne de sable argileux, Report LHM/RD/82/83, Paris School of Mines, Fontainebleau, 1982.

Faure, G, Principles of Isotope Geology, John Wiley and Sons, New York, New York, 1977.

Harada, M., P.L Chambré, M. Foglia, K. Higashi, F. Iwamoto, D. Leung, D.H. Pigford, and D. Ting, Migration of radionuclides through sorbing media, Report LBL-10500, Lawrence Berkeley Laboratory, Berkeley, California, 1980.

Ibaraki, M, and E.A. Sudicky, Colloid-facilitated contaminant transport in discretely fractured media: 1. Numerical formulation and sensitivity analysis, Water Resour. Res., 31(12), 2945-2960, 1995.

Jahnke, F.M., Electrolyte diffusion in montmorillonite engineered barriers, Ph.D. dissertation, Univ. of Calif., Berkeley, 1986.

Jahnke, F.M., and C.J. Radke, Electrolyte diffusion in compacted montmorillonite engineered barriers, in Coupled Processes Associated With Nuclear Waste Repositories, pp. 287-297, Academic Press, Orlando, 1987.

James, S.C., and C.V. Chrysikopoulos, Transport of polydisperse colloid suspensions in a single fracture, Water Resour. Res., 35(3), 707-718, 1999. 
Jensen, D.J., and C.J. Radke, Cation diffusion through compacted sodium montmorillonite at elevated temperature, J. Soil Sci., 39, 53-64, 1988.

Kersting, A., D. Efurd, D. Finnegan, D. Rokop, D. Smith, and J. Thompson, Migration of plutonium in ground water at the Nevada Test Site, Nature, 397, 56-59, 1999.

Liu, H.H., C. Doughty, and G.S. Bodvarsson, An active-fracture formulation for modeling flow and transport in fractured rocks, Water Resour. Res., 34, (10), 2633-2646, 1998.

McCarthy, J.F., and J.M. Zachara, Subsurface transport of contaminants, Env. Sci. Technol., 23(5), 496-502, 1989.

Montazer, P., andW.E. Wilson, Conceptualhydrologicmodelofflowintheunsaturatedzone, Yucca Mountain, Nevada, Resources Investigations Report 84-4355, U.S. Geological Survey, Denver, Colorado, 1984.

Moridis, G. J., Semianalytical solutions for parameter estimation in diffusion cell experiments, Water Resour. Res., 35(6), 1729-1740, 1999.

Moridis, G. J., A set of semianalytical solutions for parameter estimation in diffusion cell experiments, Report LBNL-41857, Lawrence Berkeley National Laboratory, Berkeley, Calif., 1998.

Moridis, G. J., Alternative formulations of the Laplace Transform Boundary Element (LTBE) numerical method for the solution of diffusion-type equations, in Boundary Element Technology VII, pp. 815-833, Computational Mechanics Publications, Boston, and Elsevier Applied Science, New York, 1992.

Robinson, N.I., J.M. Sharp, and I. Kreisel, Contaminant transport in sets of parellel finite fractures with fracture skins, J. Cont. Hydrol., 31, (83-109, 1998.

Saltelli,A., A. Avogadro,andG . Bidoglio,Americiumfiltrationinglauconiticsandcolumns, Nuclear Technol., 67, 245-254, 1984.

Skagius, K., and I. Neretnieks, Measurements of cesium and strontium diffusion in biotite gneiss, Water Resour. Res., 24(1), 75-84, 1988. 
Sudicky, E.A., The Laplace transform Galerkin technique for efficient time-continuous solution of solute transport in double-porosity media, Geoderma, 46209-232, 1990.

Sudicky, E.A., and E.O. Frind, Contaminant transport in fractured porous media: analytical solution for a two-member decay chain in a single fracture, Water Resour. Res., 20(7), $1021-1027,1984$.

Sudicky, E.A., and E.O. Frind, Contaminant transport in fractured porous media: analytical solutions for a system of parallel fractures, WaterResour. Res., 18(6), 1634-1642, 1982.

Tang, D.H., E.O. Frind, and E.A. Sudicky, Contaminant transport infractured porous media: Analytical solution for a single fracture, WaterResour. Res., 17(3), 555-564, 1981.

Tien, C., R.M. Turian, and H. Pendse, Simulation of the dynamic behavior of deep bed filters, J. AIChE, 25(3), 1105-1112, 1979.

Yao, K.-M., M.T. Habibian, and C.R. O'Melia, Water and waste water filtration concepts and applications, Environ. Sci. Technol., 5, 1105-1112, 1971. 
Table 1. Input parameters in Test FS1

\begin{tabular}{|c|c|}
\hline Parameters & Values \\
\hline Water saturation $S$ & 1 \\
\hline PM grain density $\rho$ & $2600 \mathrm{~kg} / \mathrm{m}^{3}$ \\
\hline$D_{0}$ & $1.6 \times 10^{-9} \mathrm{~m}^{2} / \mathrm{s}$ \\
\hline Fracture aperture $2 b$ & $10^{-4} \mathrm{~m}$ \\
\hline Fracture $S$ & 1 \\
\hline Fracture $\phi$ & 1 \\
\hline Fracture $\tau$ & 1 \\
\hline Fracture $K_{d}$ & $0 \mathrm{~m}^{3} / \mathrm{kg}$ \\
\hline Longitudinal dispersivity $\alpha_{L}$ in the fracture & $0.1 \mathrm{~m}$ \\
\hline Fracture flow velocity $V$ & $0.1 \mathrm{~m} /$ day \\
\hline Matrix block width $2 X$ & $0.5 \mathrm{~m}$ \\
\hline Matrix $S$ & 1 \\
\hline Matrix $\phi$ & 0.01 \\
\hline Matrix $\tau$ & 0.1 \\
\hline Matrix $K_{d}$ & $0 \mathrm{~m}^{3} / \mathrm{kg}$ \\
\hline Radionuclide $T_{1 / 2}$ & 12.35 years (tritium) \\
\hline$Z_{1}, Z_{2}, Z_{3}($ for $N=3)$ & $1 \mathrm{~m}, 9 \mathrm{~m}, \infty$ \\
\hline
\end{tabular}


Table 2. Input parameters in Tests FC1 and FC2

\begin{tabular}{|c|c|}
\hline Parameters & Values \\
\hline Colloid diameter $d_{c}$ & $10^{-6} \mathrm{~m}$ \\
\hline Colloid density $\rho_{C}$ & $10^{3} \mathrm{~kg} / \mathrm{m}^{3}$ \\
\hline Temperature $\mathcal{T}$ & $293.15^{\circ} \mathrm{K}$ \\
\hline Fracture aperture $2 b$ & $1.25 \times 10^{-4} \mathrm{~m}$ \\
\hline$S^{f}$ & 1 \\
\hline$\phi^{f}$ & 1 \\
\hline$\tau^{f}$ & 1 \\
\hline$\eta_{d}^{f}$ & $10^{-10} \mathrm{~m}$ \\
\hline Fracture $\kappa^{-}$ & 0 year $^{-1}$ \\
\hline Fracture flow velocity $V$ & $1 \mathrm{~m} /$ year \\
\hline Longitudinal dispersivity $\alpha_{L}$ in the fracture & $0.24998645 \mathrm{~m}$ \\
\hline$S^{m}$ & 1 \\
\hline$\phi^{m}$ & 0.3 \\
\hline$\tau^{m}$ & 0.8 \\
\hline Matrix $\kappa^{-}$ & 0 year $^{-1}$ \\
\hline Matrix $\kappa^{+}($Test FC1) & $0 \mathrm{~m}^{3} / \mathrm{kg} /$ year \\
\hline Matrix $\kappa^{+}$(Test FC2) & $5 \times 10^{-5} \mathrm{~m}^{3} / \mathrm{kg} /$ year \\
\hline$Z_{1}, Z_{2}, Z_{3}($ for $N=3)$ & $0.5 \mathrm{~m}, 0.5 \mathrm{~m}, \infty$ \\
\hline
\end{tabular}


Table 3. Input parameters in Tests PS1 to PS4

\begin{tabular}{|c|c|}
\hline Parameters & Values \\
\hline$\rho$ & $2600 \mathrm{~kg} / \mathrm{m}^{3}$ \\
\hline$D_{0}$ & $5 \times 10^{-2} \mathrm{~m}^{2} /$ day \\
\hline$S$ & 1 \\
\hline$\phi$ & 0.1 \\
\hline$\tau$ & 1 \\
\hline$V$ & $0.1 \mathrm{~m} / \mathrm{day}$ \\
\hline$K_{d}$ (Tests PS1 and PS4) & $0 \mathrm{~m} / \mathrm{kg}$ \\
\hline$K_{d}$ (Tests PS2 and PS3) & $4.2735042 \times 10^{-5} \mathrm{~m}^{3} / \mathrm{kg}$ \\
\hline$T_{1 / 2}$ (Tests PS1 and PS2) & $\infty(\mathrm{stable}$ isotopes) \\
\hline$T_{1 / 2}$ (Tests PS3 and PS4) & 100 days \\
\hline$Z_{1}, Z_{2}, Z_{3}$ (for $\left.N=3\right)$ & $10 \mathrm{~m}, 10 \mathrm{~m}, \infty$ \\
\hline
\end{tabular}


Table 4. Input parameters in Test PS5

\begin{tabular}{|c|c|}
\hline Parameters & Values \\
\hline$\rho$ & $2600 \mathrm{~kg} / \mathrm{m}^{3}$ \\
\hline$D_{0}$ & $1000 \mathrm{~m}^{2} /$ year \\
\hline$S$ & 1 \\
\hline$\phi$ & 0.3 \\
\hline$\tau$ & 1 \\
\hline$V$ & $100 \mathrm{~m} / \mathrm{year}$ \\
\hline$K_{d}$ for ${ }^{234} \mathrm{U}$ & $1.64819 \mathrm{~m}^{3} / \mathrm{kg}$ \\
\hline$K_{d}$ for ${ }^{230} \mathrm{Th}$ & $8.24159 \mathrm{~m}^{3} / \mathrm{kg}$ \\
\hline$K_{d}$ for ${ }^{226} \mathrm{Ra}$ & $8.22528 \times 10^{-2} \mathrm{~m}{ }^{3} / \mathrm{kg}$ \\
\hline$T_{1 / 2}$ of ${ }^{234} \mathrm{U}$ & $2.45 \times 10^{5}$ years \\
\hline$T_{1 / 2}$ of ${ }^{230} \mathrm{Th}$ & $7.54 \times 10^{4}$ years \\
\hline$T_{1 / 2}$ of ${ }^{226} \mathrm{Ra}$ & $1.60 \times 10^{3}$ years \\
\hline$Z_{1}, Z_{2}, Z_{3}($ for $N=3)$ & $50 \mathrm{~m}, 150 \mathrm{~m}, \infty$ \\
\hline
\end{tabular}


Table 5. Input parameters in Tests PC1 to PC3

\begin{tabular}{|c|c|}
\hline Parameters & Values \\
\hline$d_{c}$ & $10^{-3} \mathrm{~m}$ \\
\hline$\rho_{c}$ & $10^{3} \mathrm{~kg} / \mathrm{m}^{3}$ \\
\hline $\mathcal{T}$ & $293.15^{\circ} \mathrm{K}$ \\
\hline$S$ & 1 \\
\hline$\phi$ & 0.3 \\
\hline$\tau$ & 1 \\
\hline$U(=\phi V)$ & $2 \mathrm{~m} /$ day \\
\hline$\alpha_{L}$ & $0.15 \mathrm{~m}$ \\
\hline$\epsilon$ in PC1 & $30 \mathrm{~m}^{-1}$ \\
\hline$\epsilon$ in $\mathrm{PC} 2$ & $100 \mathrm{~m}^{-1}$ \\
\hline$\epsilon$ in PC3 & $3000 \mathrm{~m}^{-1}$ \\
\hline$Z_{1}, Z_{2}, Z_{3}$ (for $\left.N=3\right)$ & $0.1 \mathrm{~m}, 0.1 \mathrm{~m}, \infty$ \\
\hline
\end{tabular}

Table 6. Radionuclide properties used in the transport simulations of Section 9

\begin{tabular}{|c|c|c|}
\hline Radionuclide & $D_{0}\left(\mathrm{~m}^{2} / \mathrm{s}\right)$ & $\lambda=\frac{\ln 2}{T_{1 / 2}}(1 / \mathrm{s})$ \\
\hline${ }^{3} \mathrm{H}$ & $1.60 \times 10^{-9}$ & $1.778 \times 10^{-9}$ \\
\hline${ }^{99} \mathrm{Tc}$ & $4.55 \times 10^{-10}$ & $1.031 \times 10^{-13}$ \\
\hline${ }^{237} \mathrm{~Np}$ & $7.12 \times 10^{-10}$ & $1.026 \times 10^{-14}$ \\
\hline${ }^{239} \mathrm{Pu}$ & $6.08 \times 10^{-10}$ & $9.114 \times 10^{-13}$ \\
\hline${ }^{23.5} \mathrm{U}$ & $6.08 \times 10^{-10}$ & $3.1023 \times 10^{-17}$ \\
\hline${ }^{231} \mathrm{~Pa}$ & $6.08 \times 10^{-10}$ & $6.7583 \times 10^{-13}$ \\
\hline
\end{tabular}


Table 7. Layer geometry in Case 1-a of Problem 1

\begin{tabular}{|c|c|c|c|}
\hline Layer \# & Type & Parameter & Value \\
\hline 1 & FM & $Z$ & $5 \mathrm{~m}$ \\
& & $X$ & $0.25 \mathrm{~m}$ \\
& & $b$ & $5 \times 10^{-5} \mathrm{~m}$ \\
\hline 2 & FI & $b$ & $5 \times 10^{-5} \mathrm{~m}$ \\
\hline 3 & FM & $Z$ & $10 \mathrm{~m}$ \\
& & $X$ & $0.25 \mathrm{~m}$ \\
& & $b$ & $5 \times 10^{-5} \mathrm{~m}$ \\
\hline 4 & FI & $b$ & $5 \times 10^{-5} \mathrm{~m}$ \\
\hline 5 & FM & $Z$ & $\infty \mathrm{m}$ \\
& & $X$ & $0.25 \mathrm{~m}$ \\
& & $b$ & $5 \times 10^{-5} \mathrm{~m}$ \\
\hline
\end{tabular}


Table 8. Properties in Case 1-a1 of Problem 1

\begin{tabular}{|c|c|c|}
\hline Layer \# & Parameters & Values \\
\hline $1,3,5$ & $\begin{array}{c}\alpha_{L} \\
\phi^{m} \\
\tau^{m}=\tau_{p}^{m}=\tau_{i}^{m}=\tau_{s}^{m} \\
\phi^{f} \\
\tau^{f}=\tau_{p}^{f}=\tau_{i}^{f}=\tau_{s}^{f} \\
K_{i}^{m}=K_{i}^{f} \\
S_{w}^{m}=S_{w}^{f}\end{array}$ & $\begin{array}{c}0.1 \mathrm{~m} \\
0.01 \\
0.1 \\
1 \\
1 \\
1 \\
1\end{array}$ \\
\hline 2,4 & $\begin{array}{c}\alpha_{L} \\
\phi^{m} \\
\tau^{m}=\tau_{p}^{m}=\tau_{i}^{m}=\tau_{s}^{m} \\
\phi^{f} \\
\tau^{f}=\tau_{p}^{f}=\tau_{i}^{f}=\tau_{s}^{f} \\
K_{i}^{m}=K_{i}^{f} \\
S^{m}=S^{f}\end{array}$ & $\begin{array}{c}0.1 \mathrm{~m} \\
0.01 \\
0.1 \\
1 \\
1 \\
1 \\
1\end{array}$ \\
\hline
\end{tabular}

Table 9. Transfer coefficients in Problem S-1

\begin{tabular}{|c|c|}
\hline Radionuclide & $K_{d}$ \\
\hline${ }^{3} \mathrm{H}$ & $K_{d}^{m}=0 \mathrm{~m}^{3} / \mathrm{kg}$ \\
${ }^{3} \mathrm{H}$ & $K_{d}^{f}=0 \mathrm{~m}$ \\
\hline${ }^{237} \mathrm{~Np}$ & $K_{d}^{m}=10^{-3} \mathrm{~m}^{3} / \mathrm{kg}$ \\
${ }^{237} \mathrm{~Np}$ & $K_{d}^{f}=5 \times 10^{-7} \mathrm{~m}$ \\
\hline${ }^{239} \mathrm{Pu}$ & $K_{d}^{m}=10^{-1} \mathrm{~m}^{3} / \mathrm{kg}$ \\
${ }^{239} \mathrm{Pu}$ & $K_{d}^{f}=5 \times 10^{-5} \mathrm{~m}$ \\
\hline
\end{tabular}


Table 10. Parameter variations in the various cases of Problem 1

\begin{tabular}{|c|c|c|c|c|}
\hline Case \# & Layer \# & Type & Parameter & Value \\
\hline $1-a 2$ & 2,4 & PI & $b$ & $0.025 \mathrm{~m}$ \\
\hline $1-\mathrm{a} 3$ & 2,4 & PI & $b$ & $0.10 \mathrm{~m}$ \\
\hline \multirow[t]{2}{*}{$1-b 1$} & $1,3,5$ & FI & $X$ & $2.5 \mathrm{~m}$ \\
\hline & 2,4 & FI & $Z$ & $2.5 \mathrm{~m}$ \\
\hline \multirow[t]{3}{*}{$1-b 2$} & $1,3,5$ & FI & $X$ & $2.5 \mathrm{~m}$ \\
\hline & 2,4 & PI & $Z$ & $2.5 \mathrm{~m}$ \\
\hline & & & $b$ & $0.025 \mathrm{~m}$ \\
\hline \multirow[t]{3}{*}{$1-b 3$} & $1,3,5$ & FI & $X$ & $2.5 \mathrm{~m}$ \\
\hline & 2,4 & $\mathrm{PI}$ & $Z$ & $2.5 \mathrm{~m}$ \\
\hline & & & $b$ & $0.10 \mathrm{~m}$ \\
\hline \multirow[t]{2}{*}{$1-\mathrm{c} 1$} & $1,3,5$ & FI & $b$ & $5 \times 10^{-4} \mathrm{~m}$ \\
\hline & 2,4 & $\mathrm{FI}$ & $b$ & $5 \times 10^{-4} \mathrm{~m}$ \\
\hline \multirow[t]{2}{*}{$1-c 2$} & $1,3,5$ & FI & $X$ & $5 \times 10^{-4} \mathrm{~m}$ \\
\hline & 2,4 & PI & $b$ & $0.1 \mathrm{~m}$ \\
\hline \multirow[t]{2}{*}{$1-d 1$} & All & $\mathrm{FM}, \mathrm{FI}$ & $S_{w}^{m}$ & 0.8 \\
\hline & & & $S_{w}^{f}$ & 0.5 \\
\hline \multirow[t]{2}{*}{$1-d 2$} & All & FM, PI & $S_{w}^{m}=S_{w}^{f}$ & 0.8 \\
\hline & 2,4 & PI & $b$ & $0.1 \mathrm{~m}$ \\
\hline
\end{tabular}


Table 11. Properties and parameters in Case 2-a1

\begin{tabular}{|c|c|c|c|}
\hline Case & Layer \# & Parameter & Value \\
\hline 2-a1 & All & $\eta_{d}$ & $10^{-10} \mathrm{~m}$ \\
(Geometry & & Fracture $\kappa^{-}$ & $01 / \mathrm{s}$ \\
as in & & $\chi^{f}(5 \mathrm{~nm}$ colloid $)$ & 1 \\
Case 1-a1) & & $\chi^{f}(500 \mathrm{~nm}$ colloid $)$ & 1 \\
& & $f_{v}(5 \mathrm{~nm}$ colloid $)$ & 1 \\
& & $f_{v}(500 \mathrm{~nm}$ colloid $)$ & 1.1 \\
& & $K_{\sigma}^{m}$ & $0 \mathrm{~m}^{3} / \mathrm{kg}$ \\
& & $\chi^{m}(5 \mathrm{~nm}$ colloid $)$ & 0.95 \\
& & $\chi^{m}(500 \mathrm{~nm}$ colloid $)$ & 0.35 \\
\hline
\end{tabular}


Table 12. Properties and parameters in the cases of Problem 2

\begin{tabular}{|c|c|c|c|}
\hline Case & Layer \# (Type) & Parameter & Value \\
\hline $\begin{array}{l}\text { 2-a2 } \\
\text { (Geometry } \\
\text { as in } \\
\text { Case } 1-\mathrm{a} 2)\end{array}$ & $2,4(\mathrm{PI})$ & $\begin{array}{c}K_{\sigma}^{f} \\
\chi^{f}(5 \mathrm{~nm} \text { colloid }) \\
\chi^{f}(500 \mathrm{~nm} \text { colloid })\end{array}$ & $\begin{array}{r}0 \mathrm{~m} \\
0.95 \\
0.35\end{array}$ \\
\hline $\begin{array}{c}\text { 2-b1 } \\
\text { (As in } \\
\text { Case 2-a1) }\end{array}$ & All & $\begin{array}{l}\text { Matrix } \kappa^{+} \\
\text {Matrix } \kappa^{-}\end{array}$ & $\begin{array}{c}10^{-7} \mathrm{~m}^{3} / \mathrm{kg} / \mathrm{s} \\
10^{-6} 1 / \mathrm{s}\end{array}$ \\
\hline $\begin{array}{l}\text { 2-b2, 2-b3 } \\
\text { (Geometry }\end{array}$ & $1,3,5(\mathrm{FM})$ & $\begin{array}{l}\text { Matrix } \kappa^{+} \\
\text {Matrix } \kappa^{-}\end{array}$ & $\begin{array}{c}10^{-7} \mathrm{~m}^{3} / \mathrm{kg} / \mathrm{s} \\
10^{-6} 1 / \mathrm{s}\end{array}$ \\
\hline $\begin{array}{l}\text { as in Cases } \\
1-\mathrm{a} 2,1-\mathrm{a} 3 \\
\text { respectively) }\end{array}$ & $2,4(\mathrm{PI})$ & $\begin{array}{c}\epsilon^{f}(5 \text { and } 500 \mathrm{~nm} \text { colloid }) \\
\text { Colloid } \kappa^{-} \\
\chi^{f}(5 \mathrm{~nm} \text { colloid }) \\
\chi^{f}(500 \mathrm{~nm} \text { colloid })\end{array}$ & $\begin{aligned} & 10^{3} \\
= & 0.1 \kappa^{+} \\
& 0.95 \\
& 0.35\end{aligned}$ \\
\hline $2-\mathrm{b} 4$ & All & As in $2-\mathrm{b} 2$ but $\epsilon^{f}=10^{2}$ & \\
\hline $\begin{array}{c}2-c 1,2-c 2 \\
\text { (Geometry of } 1-b 1)\end{array}$ & All & $\begin{array}{l}\text { Properties as in Cases } \\
\text { 2-a1, 2-b1 respectively }\end{array}$ & \\
\hline $\begin{array}{l}2-c 3,2-c 4 \\
\text { (Geometry of } 1-b 2)\end{array}$ & All & $\begin{array}{l}\text { Properties as in Cases } \\
\text { 2-a1, 2-b2 respectively }\end{array}$ & \\
\hline
\end{tabular}


Table 13. Layer geometry in Problem 3

\begin{tabular}{|c|c|c|c|c|}
\hline Layer \# & Type & $Z(\mathrm{~m})$ & $X(\mathrm{~m})$ & $b(\mathrm{~m})$ \\
\hline 1 & FM & 10 & 0.5 & $10^{-4}$ \\
\hline 2 & PI & & & $5 \times 10^{-2}$ \\
\hline 3 & FM & 10 & 0.25 & $5 \times 10^{-5}$ \\
\hline 4 & PI & & & $2.5 \times 10^{-2}$ \\
\hline 5 & FM & 10 & 3 & $2 \times 10^{-4}$ \\
\hline 6 & PM & 5 & & \\
\hline 7 & FM & 15 & 0.1 & $2 \times 10^{-4}$ \\
\hline 8 & PI & & & $10^{-1}$ \\
\hline 9 & FM & 10 & 4 & $2 \times 10^{-5}$ \\
\hline 10 & FI & & & $2 \times 10^{-5}$ \\
\hline 11 & FM & 20 & 1 & $5 \times 10^{-5}$ \\
\hline 12 & PM & 5 & & $8 \times 10^{-5}$ \\
\hline 13 & FM & 30 & 6 & \\
\hline 14 & PM & $\infty$ & & \\
\hline
\end{tabular}


Table 14. Rock properties in Problem 3

\begin{tabular}{|c|c|c|c|c|c|c|}
\hline Layer \# & $\phi^{m}$ & $\tau^{m}$ & $S_{w}^{m}$ & $\phi^{f}$ & $\tau^{f}$ & $S_{w}^{f}$ \\
\hline 1 & 0.15 & 0.5 & 0.7 & 1 & 1 & 0.2 \\
\hline 2 & 0.3 & 0.3 & 1 & 0.3 & 0.3 & 0.4 \\
\hline 3 & 0.1 & 0.4 & 0.6 & 1 & 1 & 0.15 \\
\hline 4 & 0.35 & 0.3 & 1 & 0.35 & 0.3 & 0.3 \\
\hline 5 & 0.05 & 0.5 & 0.8 & 1 & 1 & 0.1 \\
\hline 6 & 0.35 & 0.8 & 0.9 & 0.35 & 0.8 & 0.9 \\
\hline 7 & 0.025 & 0.2 & 0.9 & 1 & 1 & 0.1 \\
\hline 8 & 0.2 & 0.3 & 0.9 & 0.2 & 0.3 & 0.4 \\
\hline 9 & 0.01 & 0.2 & 0.95 & 1 & 1 & 0.05 \\
\hline 10 & 0.01 & 0.2 & 0.95 & 1 & 1 & 0.05 \\
\hline 11 & 0.05 & 0.15 & 0.95 & 1 & 1 & 0.05 \\
\hline 12 & 0.1 & 0.1 & 0.9 & 0.2 & 0.1 & 0.9 \\
\hline 13 & 0.05 & 0.1 & 1 & 1 & 1 & 1 \\
\hline 14 & 0.1 & 0.1 & 1 & 0.1 & 0.1 & 1 \\
\hline
\end{tabular}


Table 15. Transfer coefficients in Problem 3

\begin{tabular}{|c|c|c|c|c|c|c|}
\hline \multirow{2}{*}{$\begin{array}{c}\text { Layer } \\
\#\end{array}$} & \multicolumn{2}{|c|}{${ }^{3} \mathrm{H}$ or ${ }^{99} \mathrm{Tc}$} & \multicolumn{2}{|c|}{${ }^{237} \mathrm{~Np}$} & \multicolumn{2}{|c|}{${ }^{239} \mathrm{Pu}$} \\
\hline & $K_{d}^{m}\left(^{*}\right)$ & $K_{d}^{f}(\dagger)$ & $K_{d}^{m}(*)$ & $K_{d}^{f}(\dagger)$ & $K_{d}^{m}(*)$ & $K_{d}^{f}(\dagger)$ \\
\hline 1 & 0 & 0 & $6 \times 10^{-4}$ & $3 \times 10^{-8}$ & $6 \times 10^{-2}$ & $3 \times 10^{-6}$ \\
\hline 2 & 0 & 0 & $8 \times 10^{-4}$ & $8 \times 10^{-4}$ & $8 \times 10^{-2}$ & $8 \times 10^{-2}$ \\
\hline 3 & 0 & 0 & $7 \times 10^{-4}$ & $3.5 \times 10^{-8}$ & $7 \times 10^{-2}$ & $3.5 \times 10^{-6}$ \\
\hline 4 & 0 & 0 & $8 \times 10^{-4}$ & $8 \times 10^{-4}$ & $8 \times 10^{-2}$ & $8 \times 10^{-2}$ \\
\hline 5 & 0 & 0 & $8 \times 10^{-4}$ & $4 \times 10^{-8}$ & $8 \times 10^{-2}$ & $4 \times 10^{-6}$ \\
\hline 6 & 0 & 0 & $10^{-4}$ & $10^{-4}$ & $10^{-2}$ & $10^{-2}$ \\
\hline 7 & 0 & 0 & $10^{-3}$ & $5 \times 10^{-7}$ & $10^{-1}$ & $5 \times 10^{-5}$ \\
\hline 8 & 0 & 0 & $8 \times 10^{-4}$ & $8 \times 10^{-4}$ & $8 \times 10^{-2}$ & $8 \times 10^{-2}$ \\
\hline 9 & 0 & 0 & $5 \times 10^{-4}$ & $2.5 \times 10^{-8}$ & $5 \times 10^{-2}$ & $2.5 \times 10^{-6}$ \\
\hline 10 & 0 & 0 & $5 \times 10^{-4}$ & $2.5 \times 10^{-8}$ & $5 \times 10^{-2}$ & $2.5 \times 10^{-6}$ \\
\hline 11 & 0 & 0 & $9 \times 10^{-4}$ & $4.5 \times 10^{-8}$ & $9 \times 10^{-2}$ & $4.5 \times 10^{-6}$ \\
\hline 12 & 0 & 0 & $10^{-3}$ & $10^{-3}$ & $10^{-1}$ & $10^{-1}$ \\
\hline 13 & 0 & 0 & $6 \times 10^{-4}$ & $3 \times 10^{-8}$ & $6 \times 10^{-2}$ & $3 \times 10^{-6}$ \\
\hline 14 & 0 & 0 & $7 \times 10^{-4}$ & $7 \times 10^{-4}$ & $7 \times 10^{-2}$ & $7 \times 10^{-2}$ \\
\hline
\end{tabular}

$(*)$ : in $\mathrm{m}^{3} / \mathrm{kg},(\dagger)$ : in $\mathrm{m}$ 
Table 16. Colloid filtration and straining parameters in Problem 5

\begin{tabular}{|c|c|c|c|c|c|c|}
\hline $\begin{array}{c}\text { Layer } \\
\text { No. }\end{array}$ & $\begin{array}{c}\text { Matrix } \kappa^{+} \\
\left(\mathrm{m}^{3} / \mathrm{kg} / \mathrm{s}\right)\end{array}$ & $\begin{array}{c}\text { Matrix } \kappa^{-} \\
(1 / \mathrm{s})\end{array}$ & $\begin{array}{c}\left(^{*}\right) \epsilon(\mathrm{m}) \\
\text { or } \eta_{d}(1 / \mathrm{m})\end{array}$ & $\begin{array}{c}\text { Fracture } \\
\kappa^{-}\end{array}$ & $\begin{array}{c}\chi^{f} \\
\chi^{f}\end{array}$ & $\begin{array}{c}\chi^{m} \\
\chi^{m}\end{array}$ \\
\hline 2 & $10^{-7}$ & $10^{-6}$ & $\eta_{d}=10^{-10}$ & 0 & - & 1 \\
\hline 3 & $10^{-7}$ & $10^{-6}$ & $\epsilon=10^{3}$ & $=0.1 \kappa^{+}$ & 0.97 & 1 \\
\hline 4 & $10^{-7}$ & $10^{-6}$ & $\eta_{d}=10^{-10}$ & 0 & 1 & 1 \\
\hline 5 & $10^{-7}$ & $10^{-6}$ & $\epsilon=10^{3}$ & $=0.1 \kappa^{+}$ & 0.97 & 1 \\
\hline 6 & $10^{-7}$ & $10^{-6}$ & $\eta_{d}=10^{-10}$ & 0 & 1 & 1 \\
\hline 7 & $10^{-8}$ & $10^{-6}$ & $\epsilon=10^{2}$ & $=0.1 \kappa^{+}$ & 0.97 & 1 \\
\hline 8 & $10^{-7}$ & $10^{-6}$ & $\eta_{d}=10^{-10}$ & 0 & 1 & 1 \\
\hline 9 & $10^{-7}$ & $10^{-6}$ & $\eta_{d}=10^{-10}$ & 0 & 1 & 1 \\
\hline 10 & $10^{-7}$ & $10^{-6}$ & $\eta_{d}=10^{-10}$ & 0 & 1 & 1 \\
\hline 11 & $10^{-7}$ & $10^{-6}$ & $\eta_{d}=10^{-10}$ & 0 & 1 & 1 \\
\hline 12 & $10^{-7}$ & $10^{-6}$ & $\epsilon=10^{3}$ & $=0.1 \kappa^{+}$ & 0.95 & 1 \\
\hline 13 & $5 \times 10^{-8}$ & $10^{-6}$ & $\eta_{d}=10^{-9}$ & 0 & 1 & 1 \\
\hline 14 & $5 \times 10^{-8}$ & $10^{-6}$ & $\epsilon=10^{3}$ & $=0.05 \kappa^{+}$ & 0.95 & 1 \\
\hline
\end{tabular}

$\left(^{*}\right)$ : The fracture $\kappa^{+}$is computed from equations (30) and (40). 
Layers 1,2,4,1: Fractured media

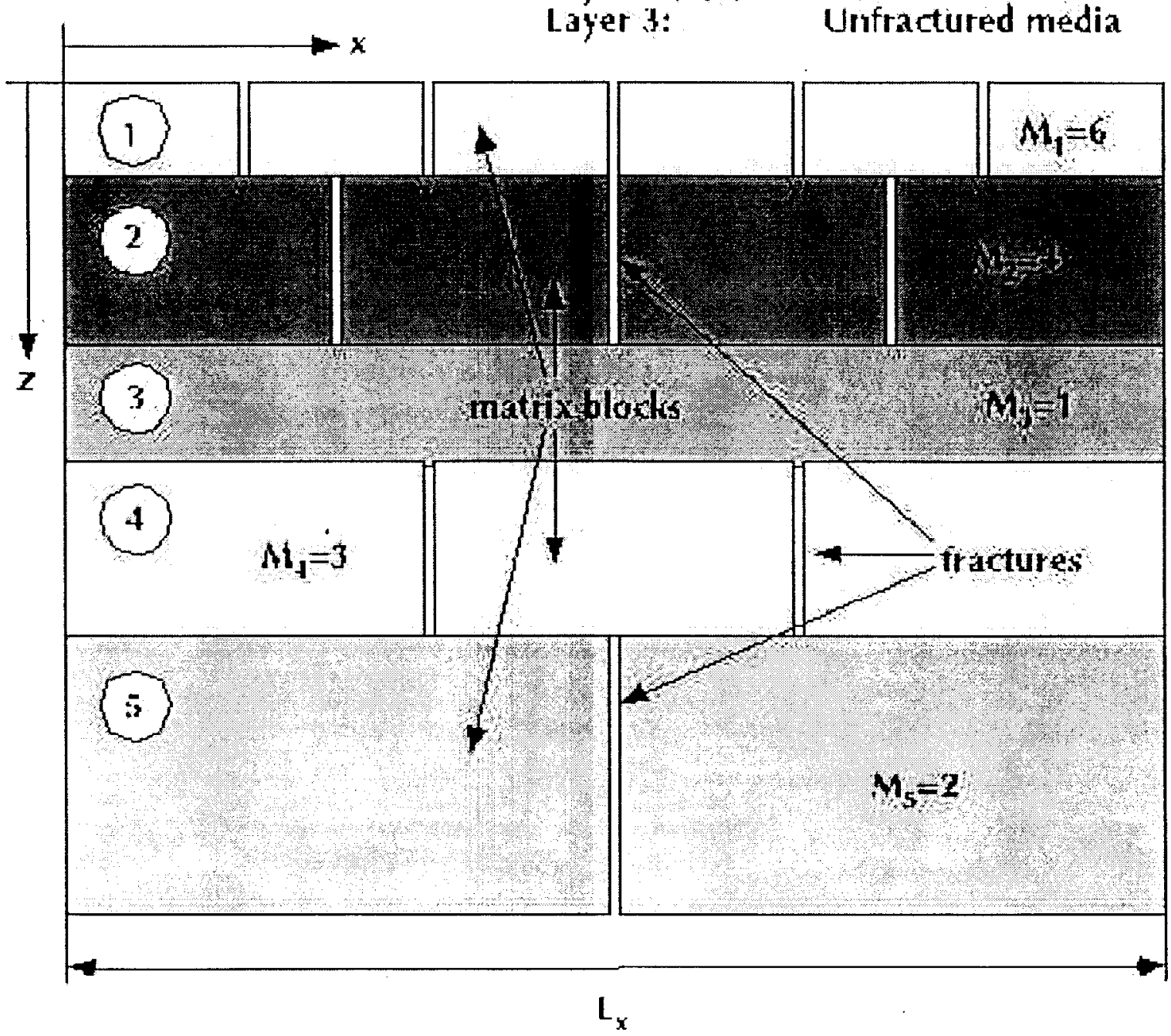

Figure 1. A variably-fractured layered geologic system. 


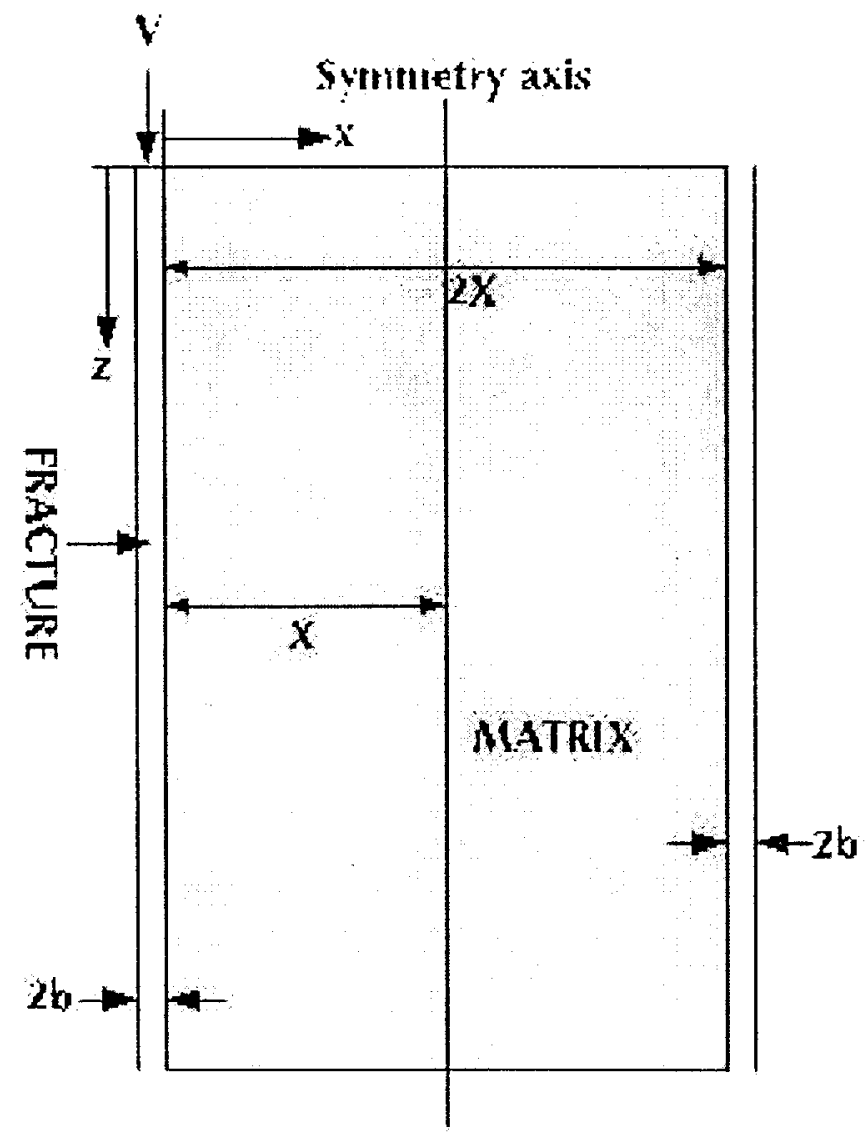

(a) Case 1

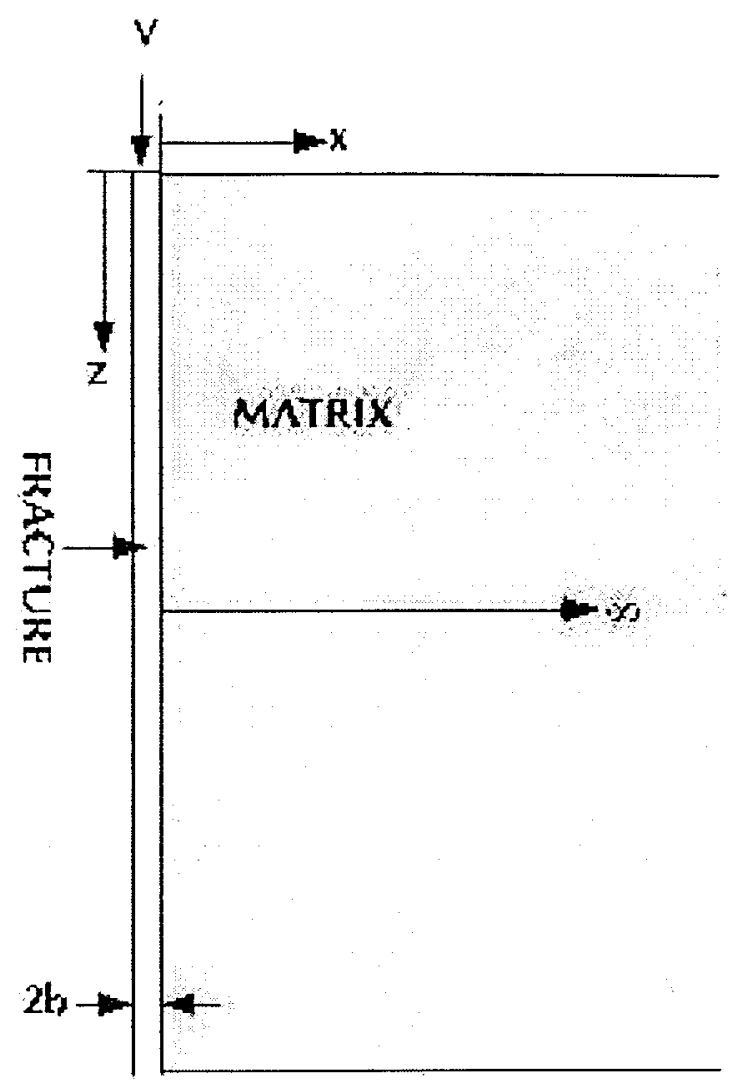

(b) Ciase 2

Figure 2. Fracture-matrix configurations and important parameters in Cases 1 and 2. 

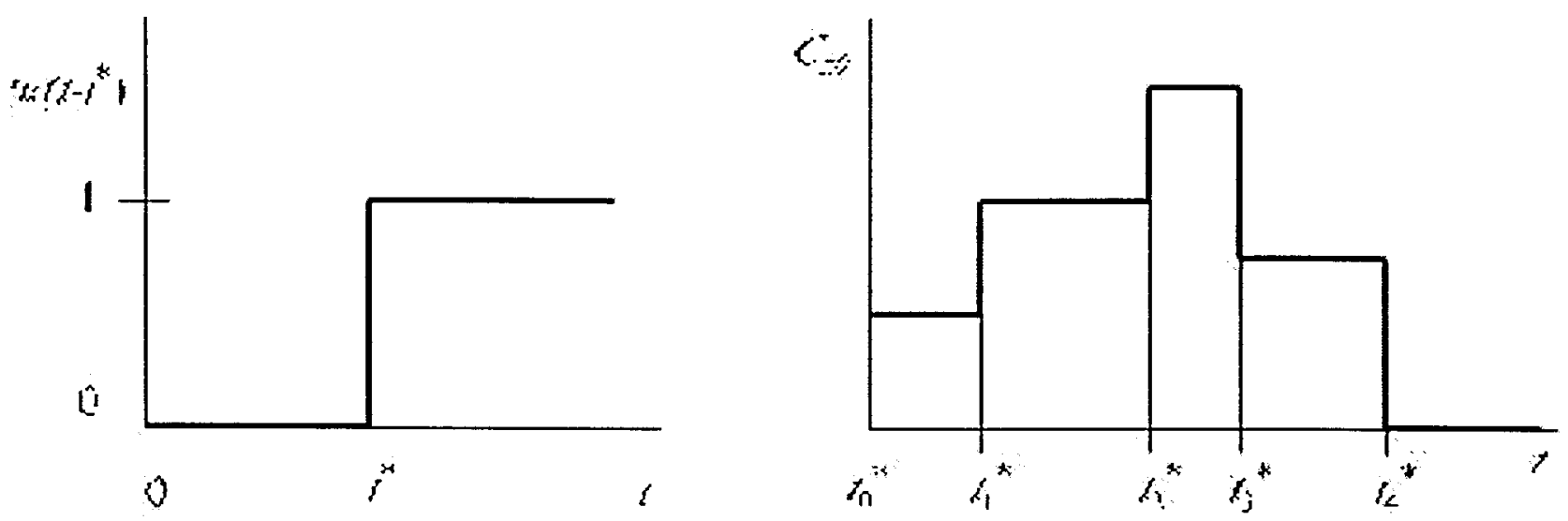

Figure 3. Unit step function and variable pulse concentration at the $z=0$ boundary. 

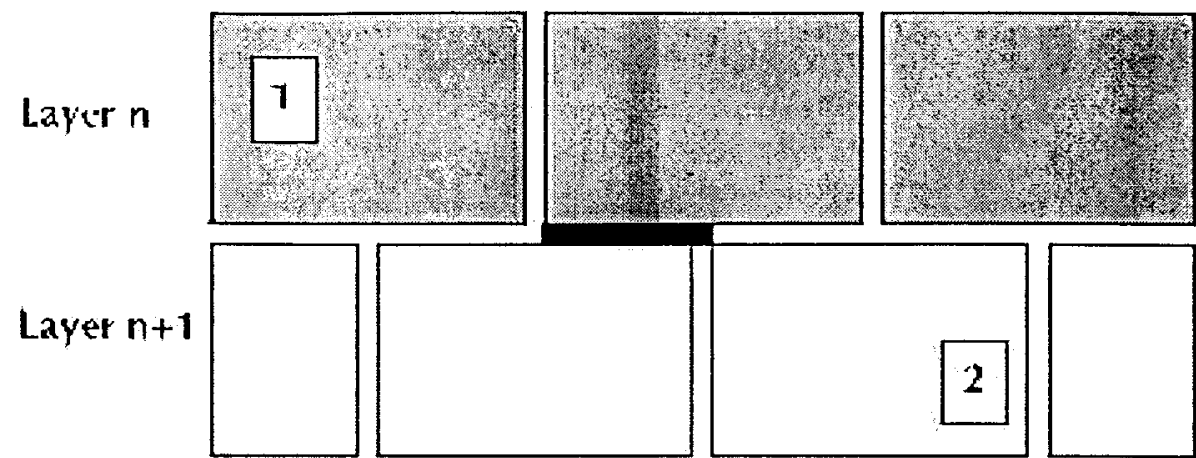

(a)

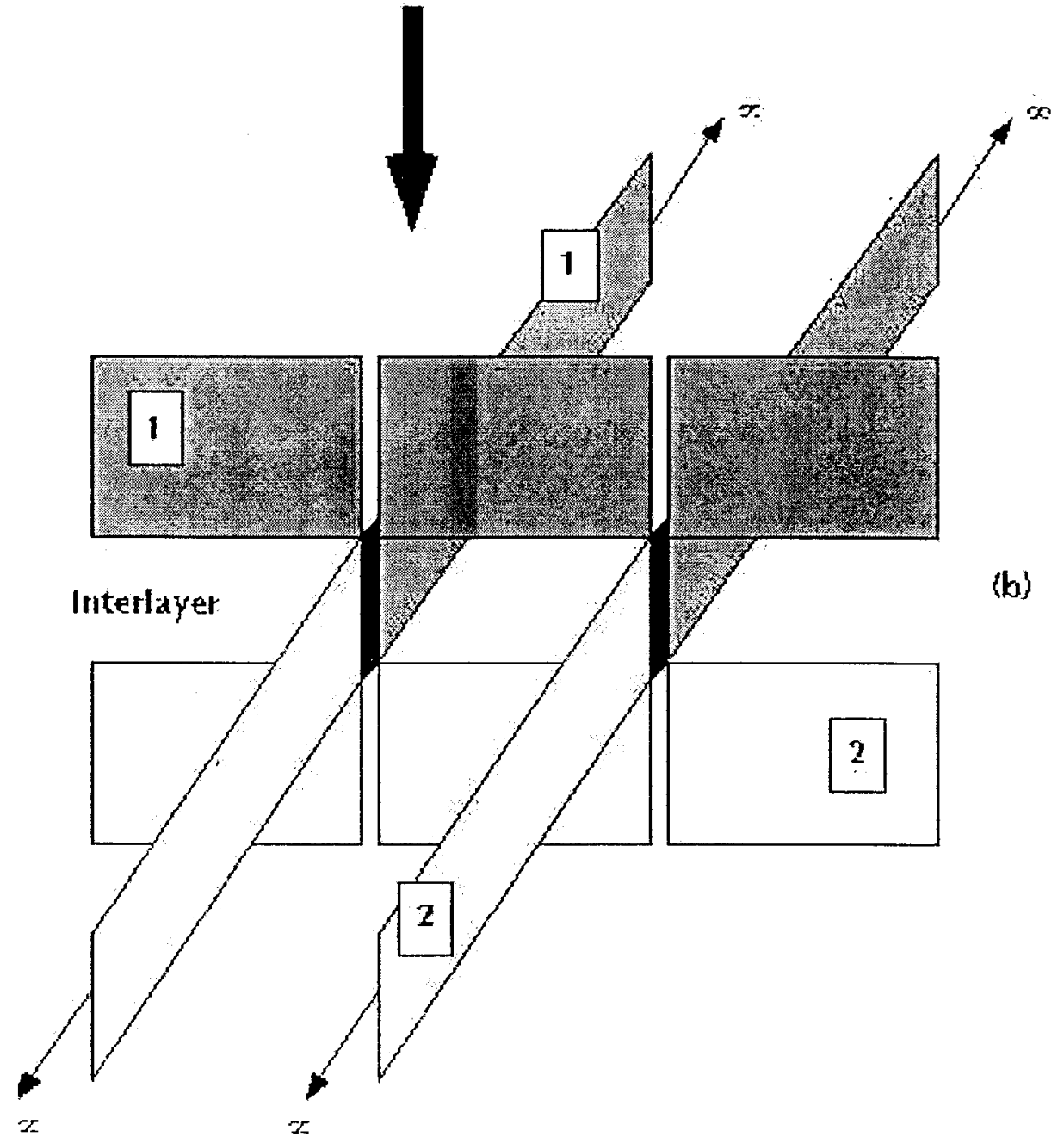

Figure 4. A graphic representation of the concept of interlayer describing the effects of fracture misalignment. The properties of layers $n$ and $n+1$ are denoted by 1 and 2 , respectively. 


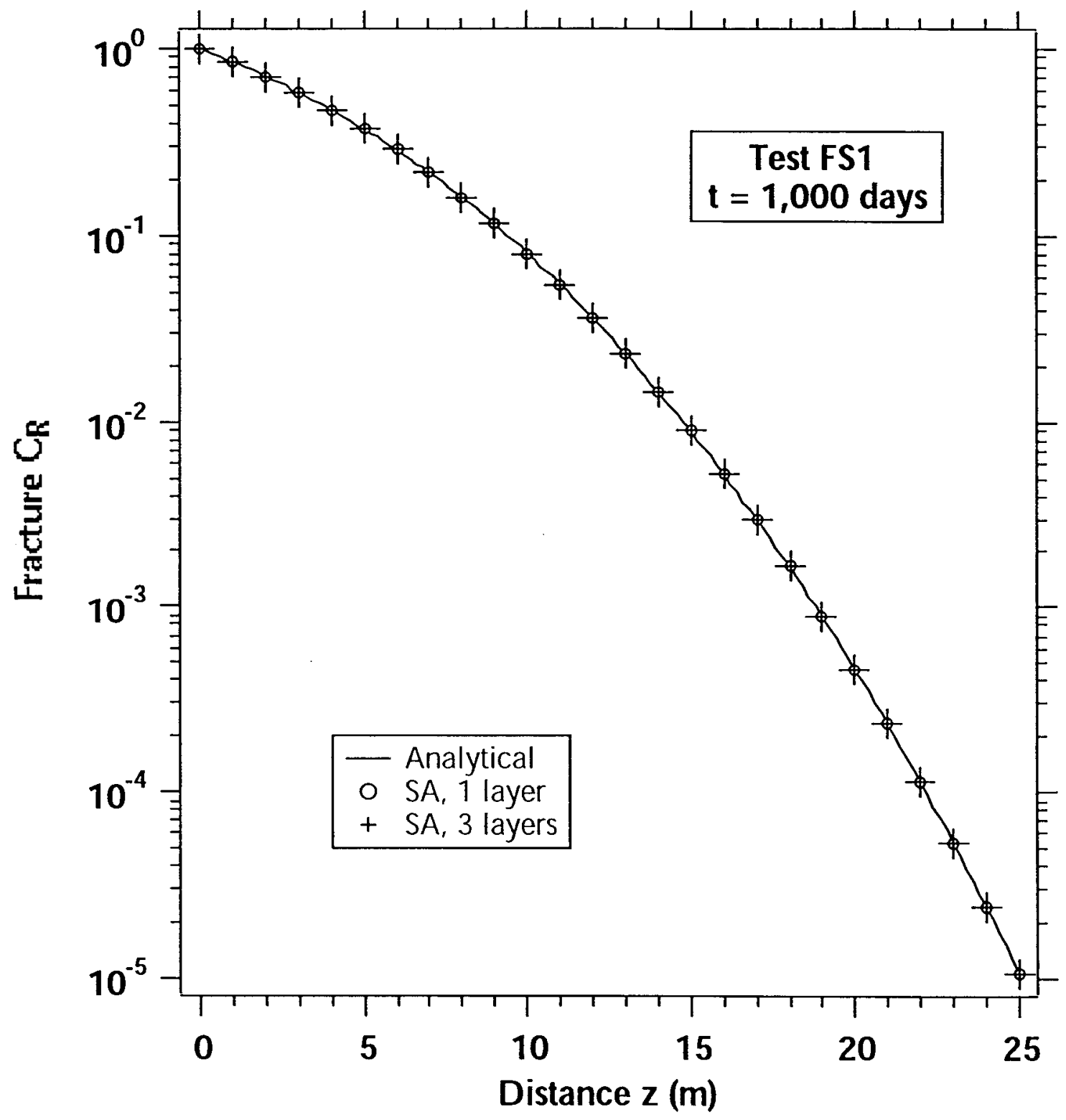

Figure 5. Comparison of the semianalytical (SA) solutions from FRACL to the analytical solution of radioactive solute transport in fractured media in Test FS1. 


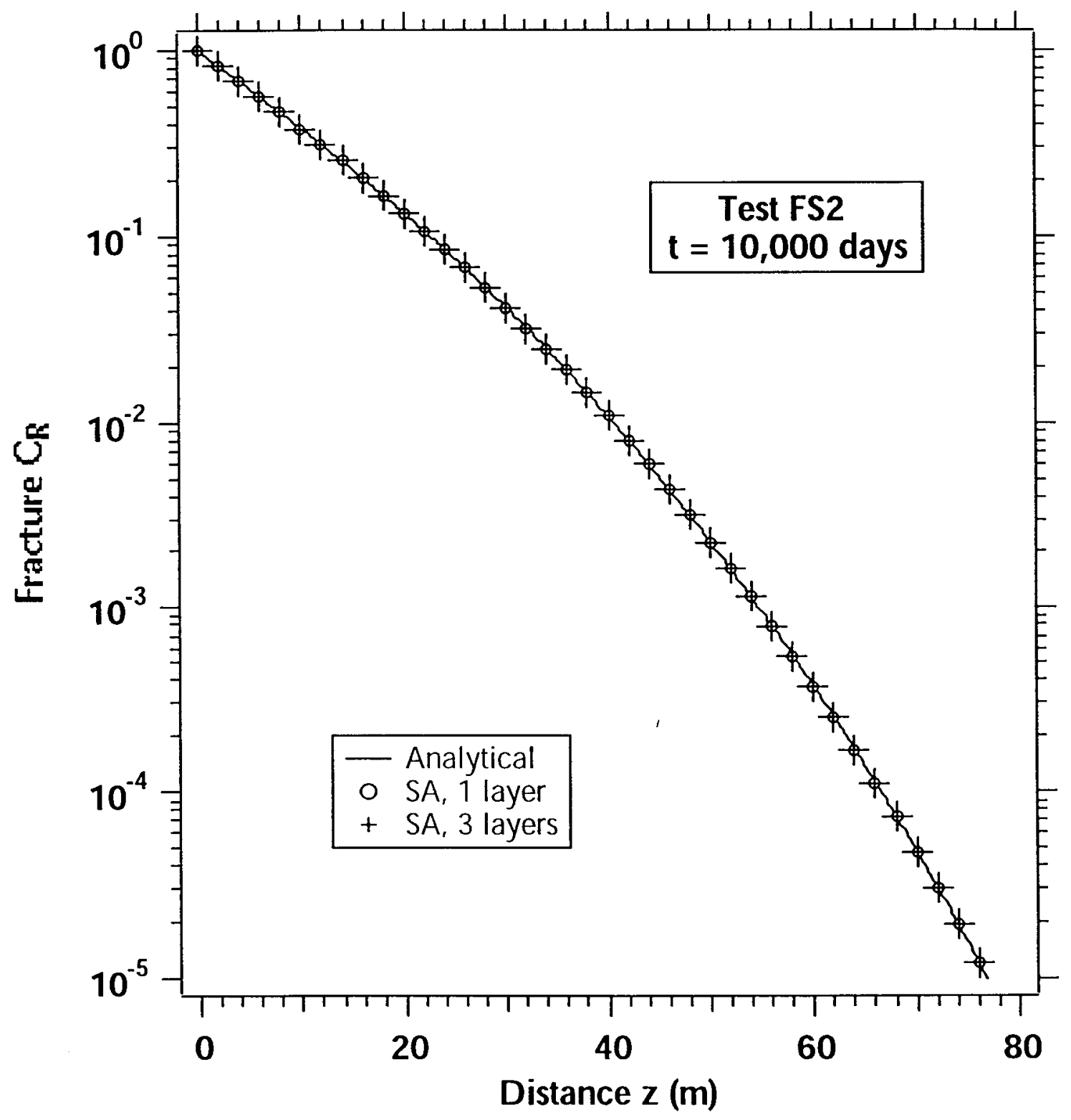

Figure 6. Comparison of the SA solutions to the analytical solution of radioactive solute transport in fractured media in Test FS2. 


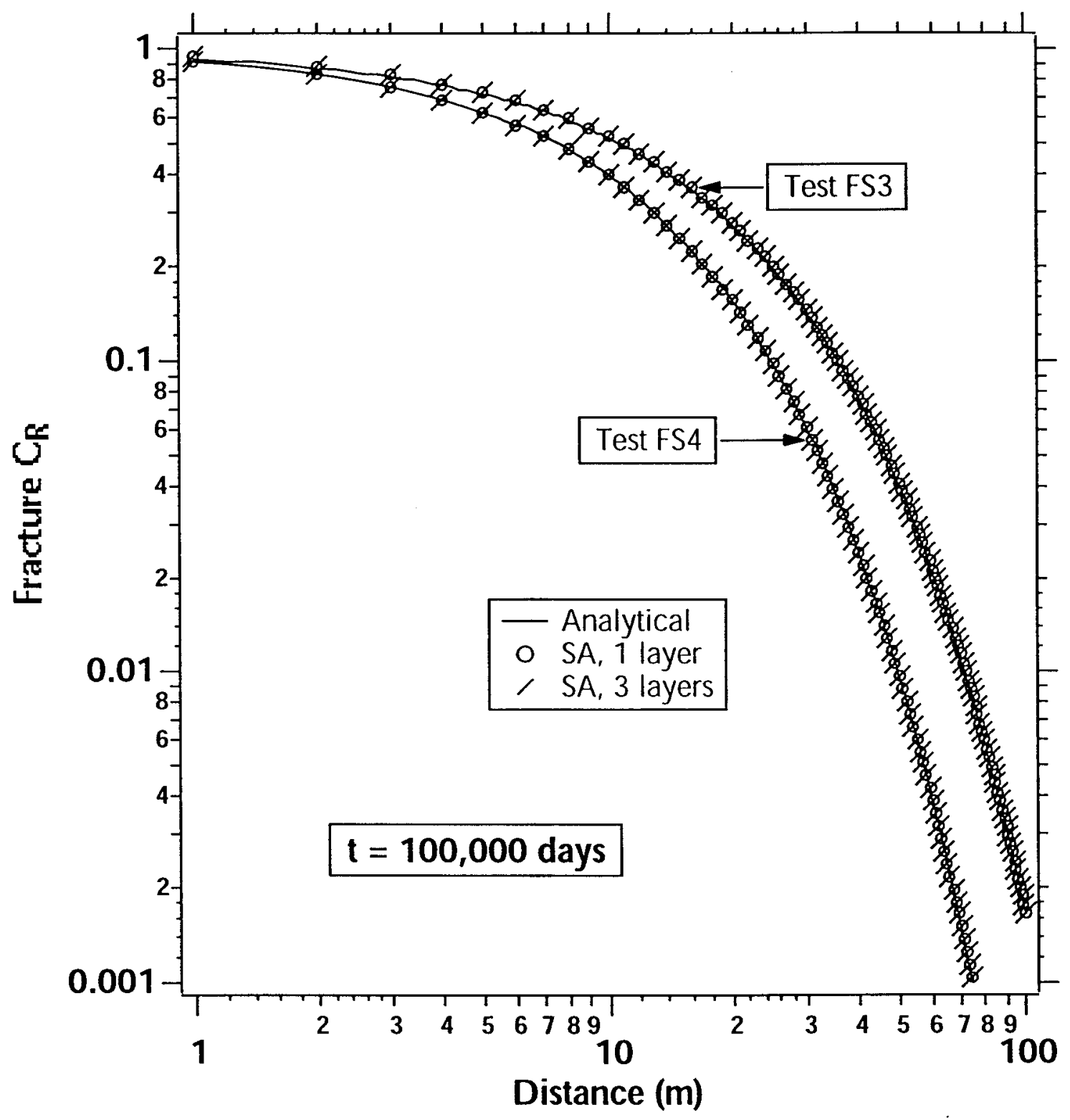

Figure 7. Comparison of the SA solutions from FRACL to the analytical solutions of radioactive solute transport in fractured media in Tests FS3 and FS4. 


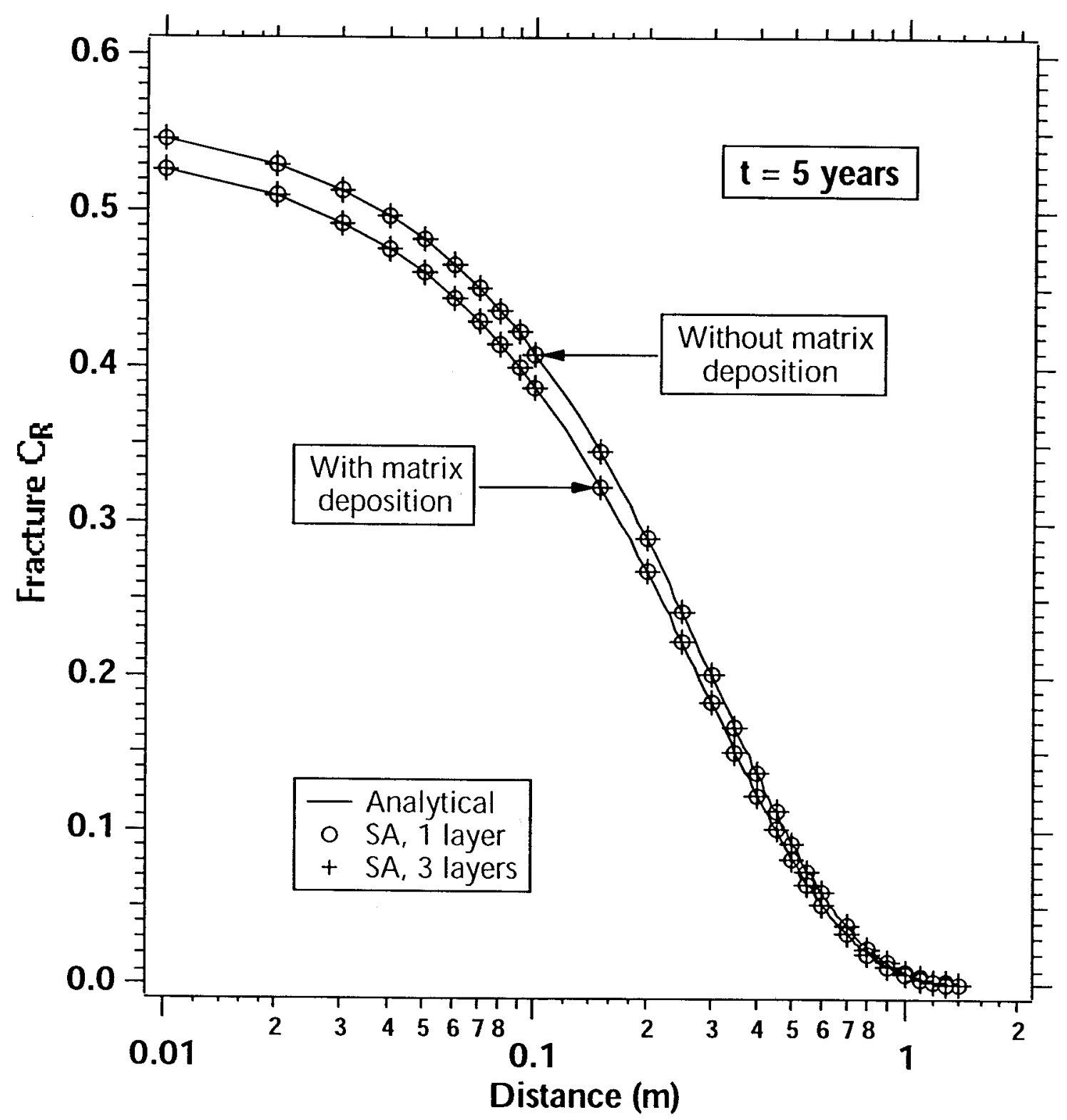

Figure 8. Comparison of the SA solutions to the analytical solutions of colloid transport in fractured media in Tests FC1 (without colloid filtration in the matrix) and FC2 (with colloid filtration in the matrix). 


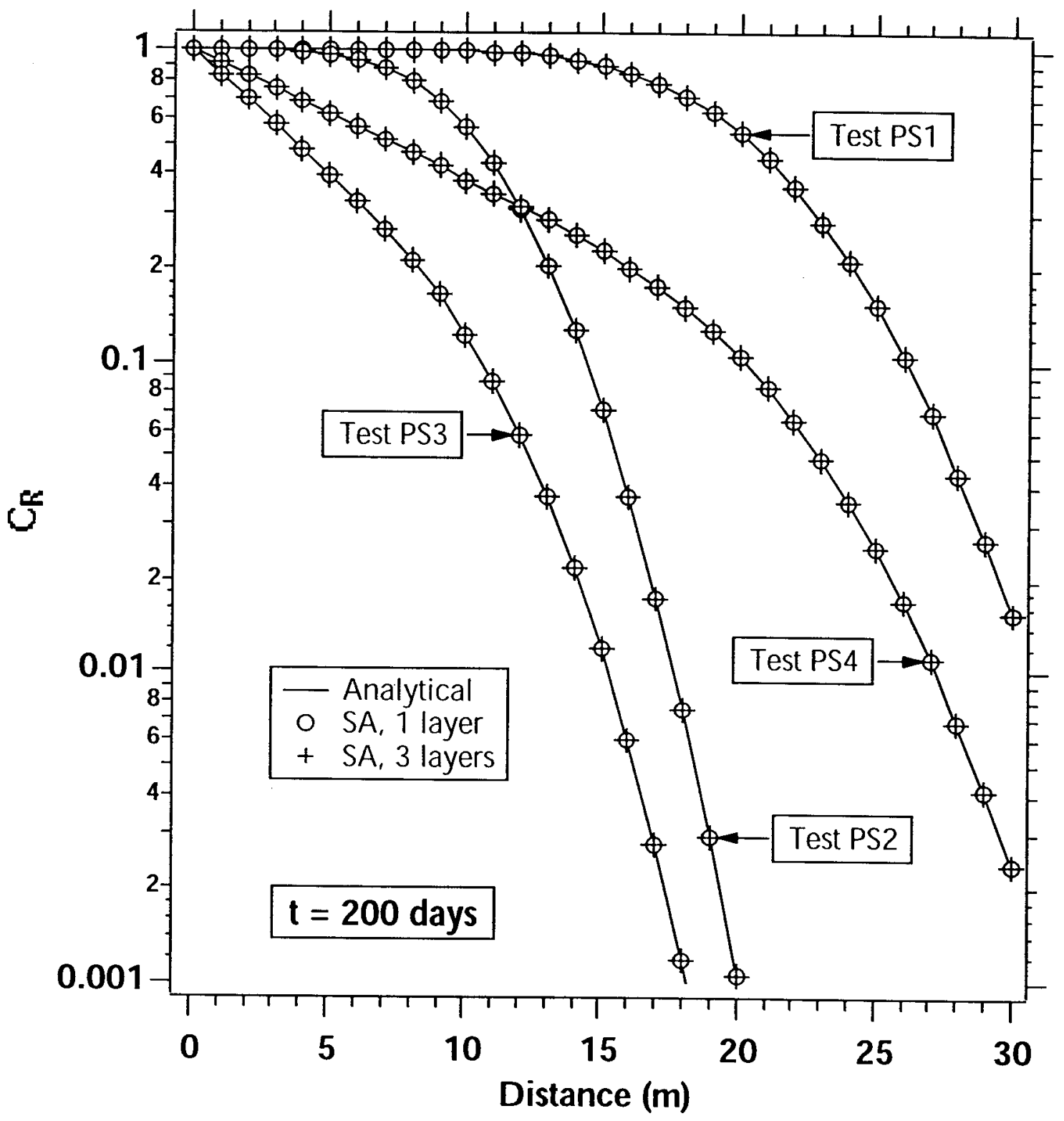

Figure 9. Comparison of the SA solutions to the analytical solutions of solute transport in porous media in Tests PSI to PS4. 


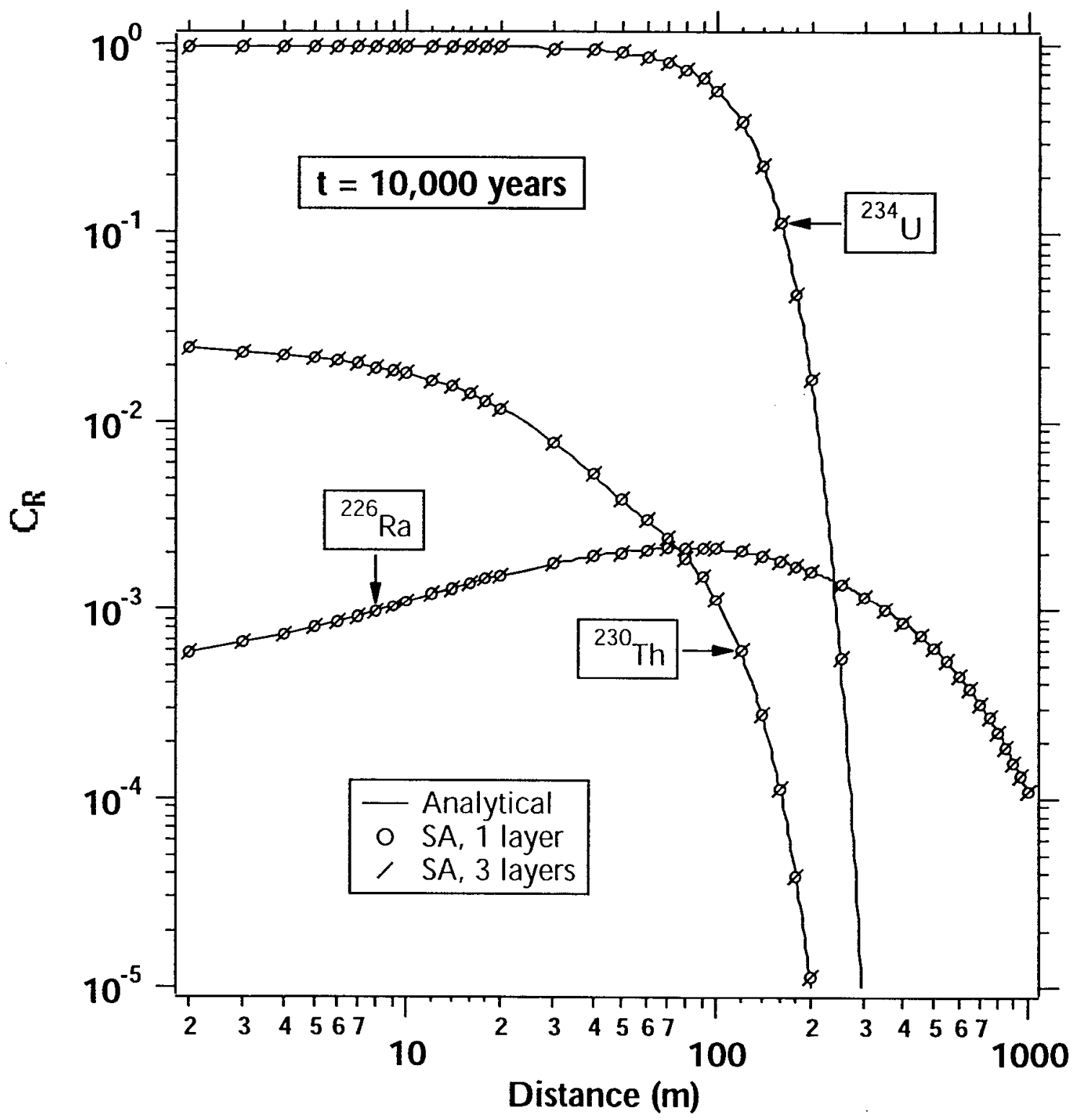

Figure 10. Comparison of the SA solutions from FRACL to the analytical solutions of solute transport of the radioactive chain ${ }^{234} \mathrm{U} \rightarrow{ }^{230} \mathrm{Th} \rightarrow{ }^{226} \mathrm{Ra}$ in porous media in Test PS5. 


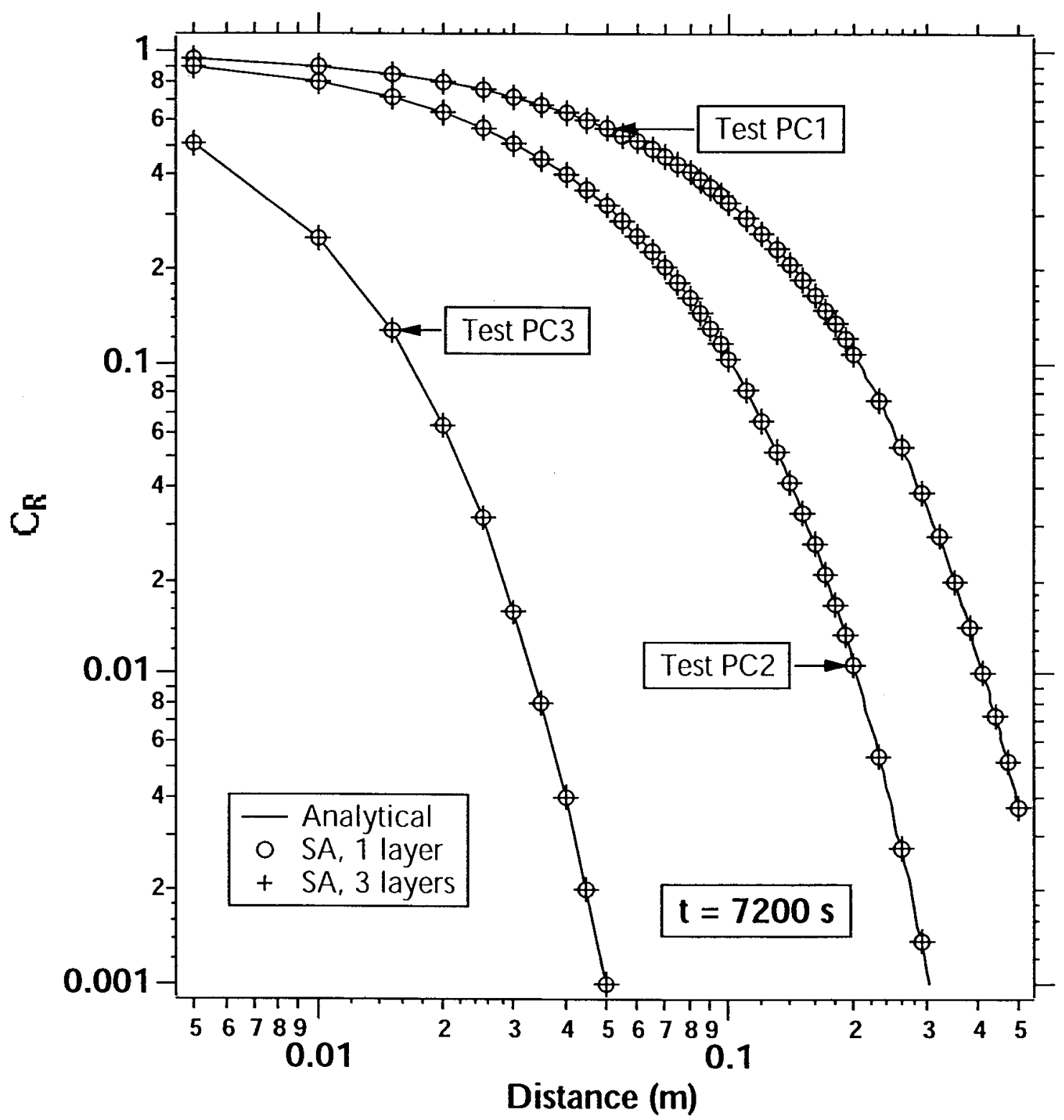

Figure 11. Comparison of the SA solutions to the analytical solutions of colloid transport in porous media in Tests PC1 to PC3. 


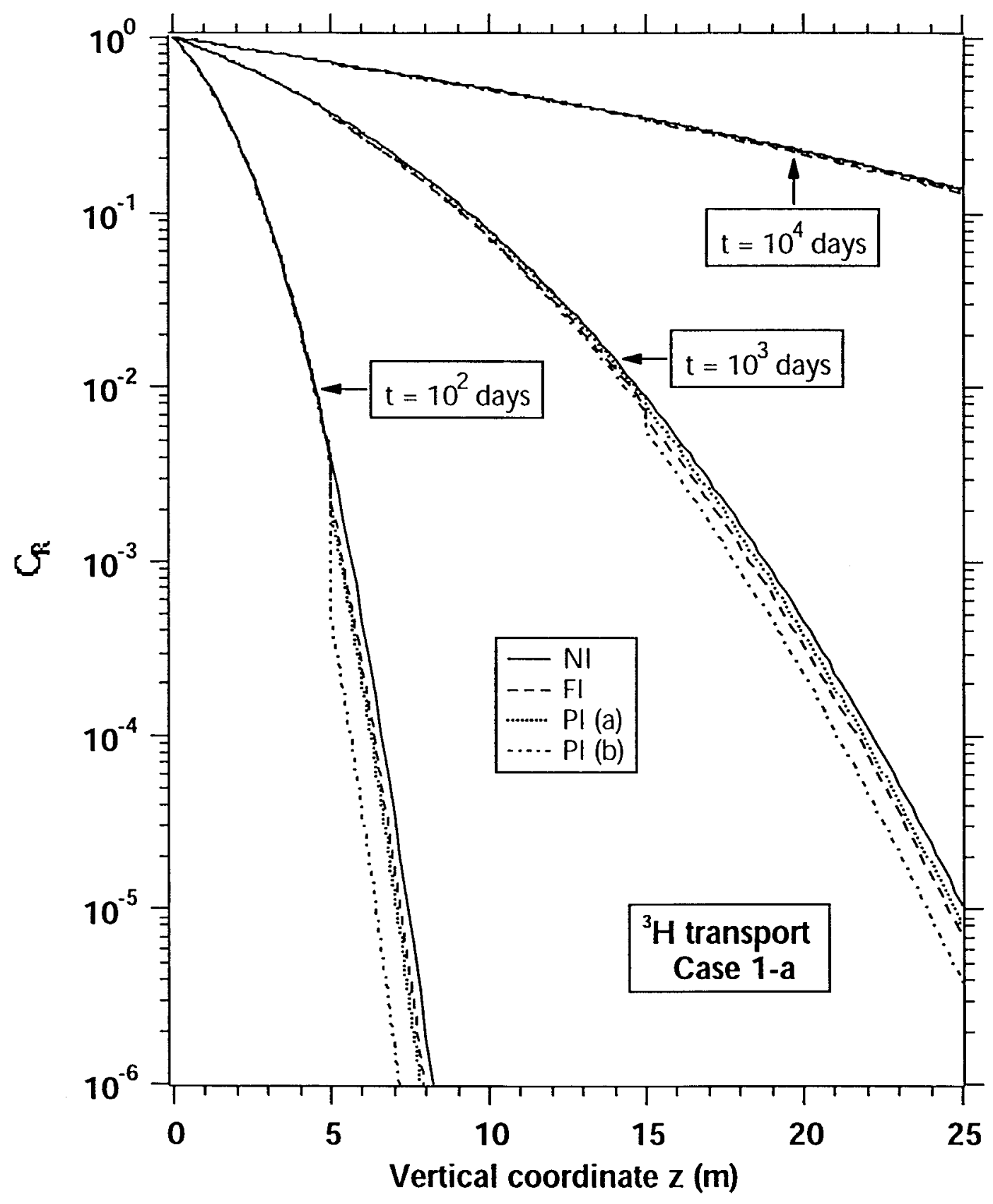

Figure 12. Effect of fracture offset (presence of interlayers) on the transport of ${ }^{3} \mathrm{H}$ through the layered fractured system of Case 1-a (NI: no interlayer, FI: fracture interlayer, PI(a): porous interlayer with $b=0.025 \mathrm{~m}, \mathrm{PI}(\mathrm{b})$ : porous interlayer with $b=0.1 \mathrm{~m})$. 


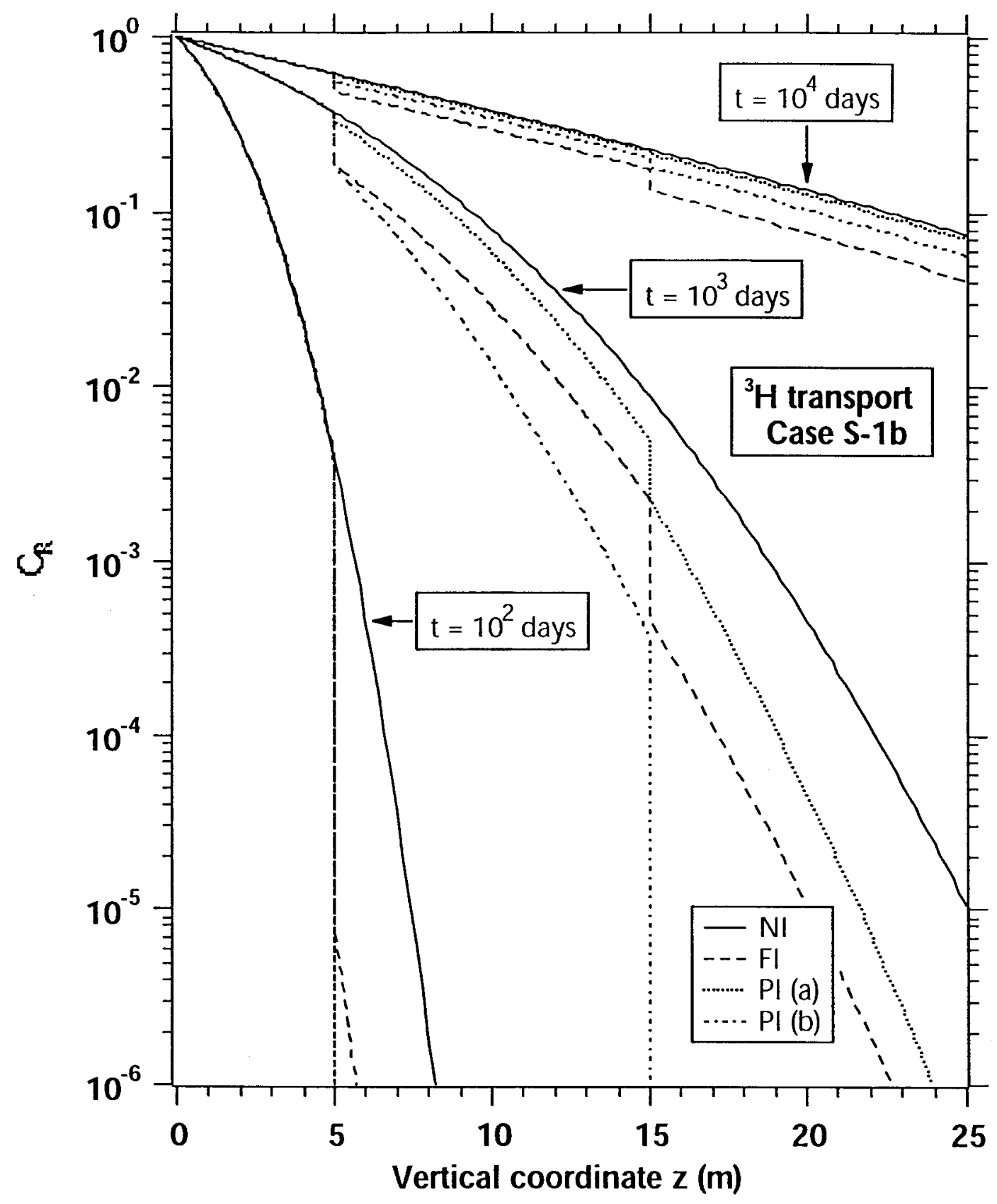

Figure 13. Combined effect of increased $X$ and fracture offset (presence of interlayers) on the transport of ${ }^{3} \mathrm{H}$ through the layered fractured system of Case 1-b (nomenclature as in Figure 12). 


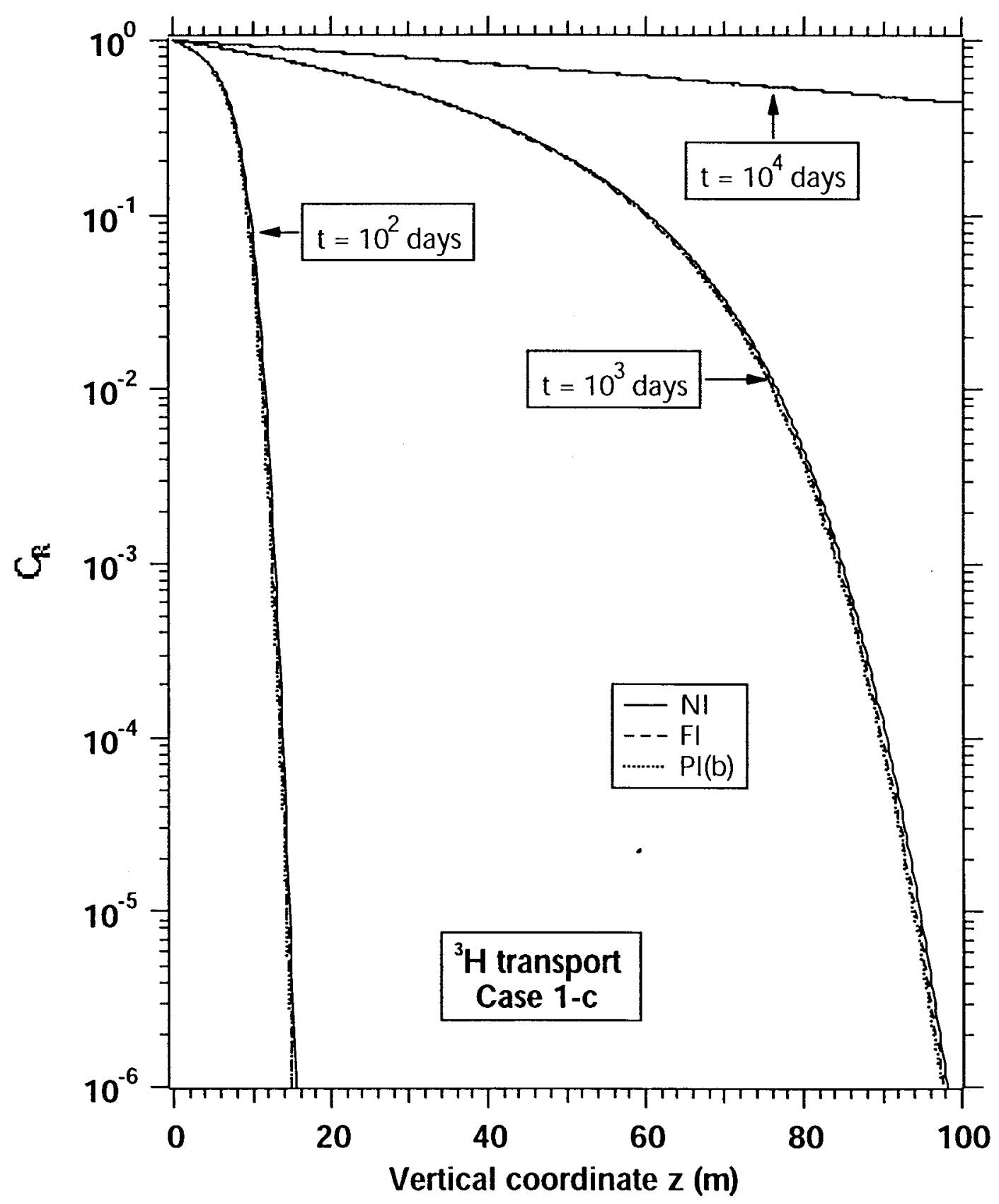

Figure 14. Combined effect of increased $b$ and fracture offset on the transport of ${ }^{3} \mathrm{H}$ through the layered fractured system of Case 1-c (nomenclature as in Figure 12). 


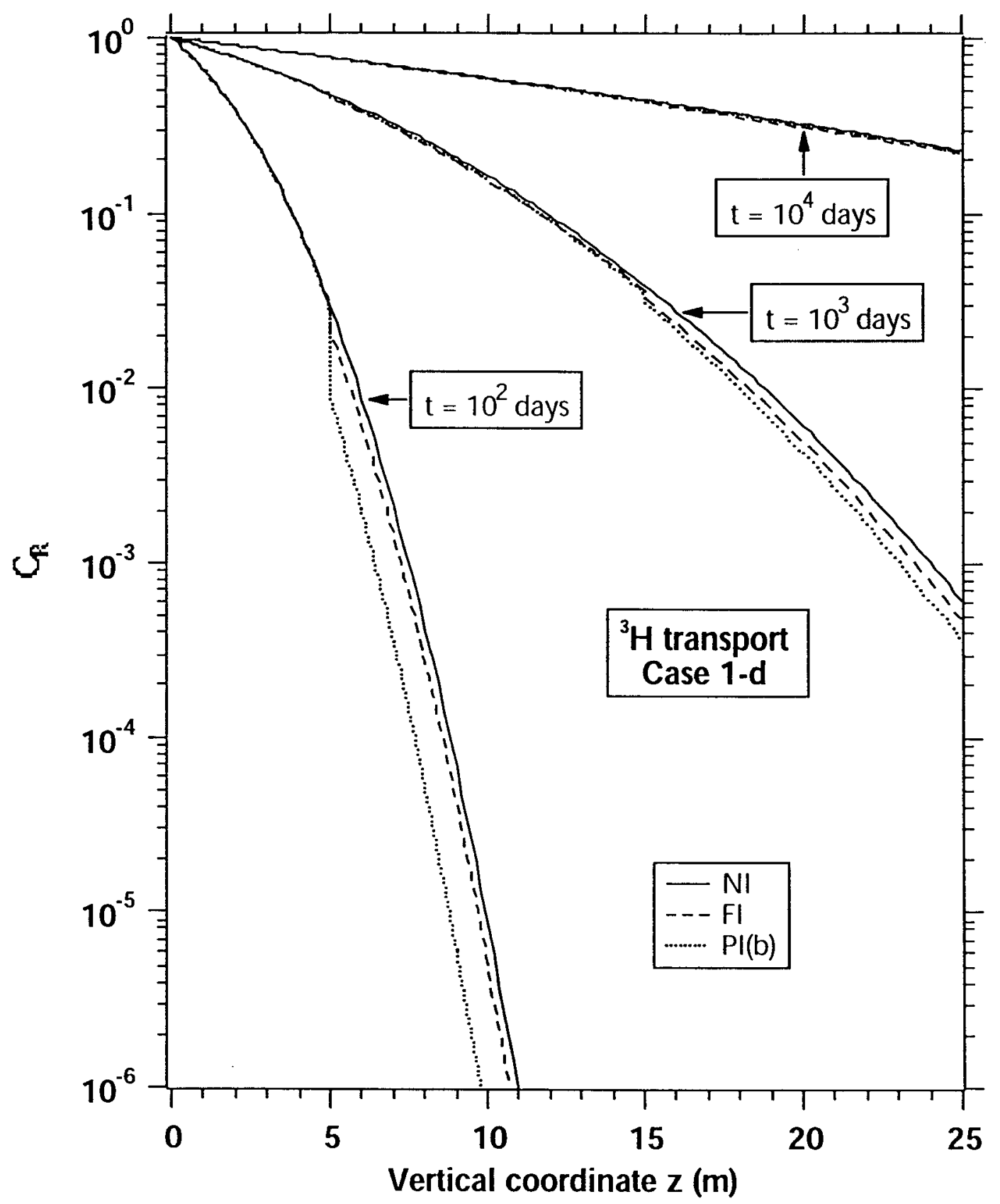

Figure 15. Combined effect of water saturation $S$ and fracture offset on the transport of ${ }^{3} \mathrm{H}$ through the layered fractured system of Case 1-d (nomenclature as in Figure 12). 


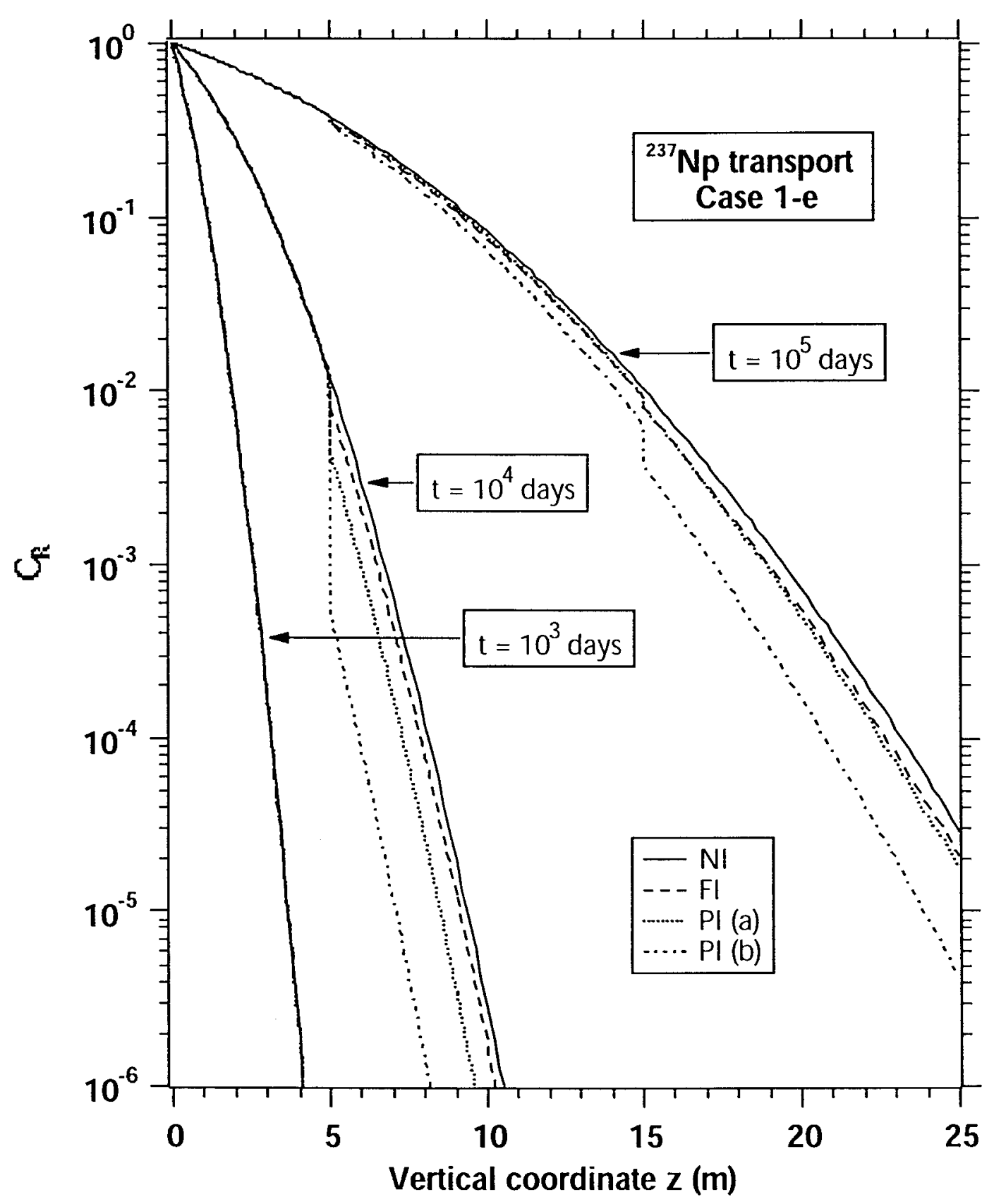

Figure 16. Effect of fracture offset (presence of interlayers) on the transport of ${ }^{237} \mathrm{~Np}$ through the layered fractured system of Case 1-e. 


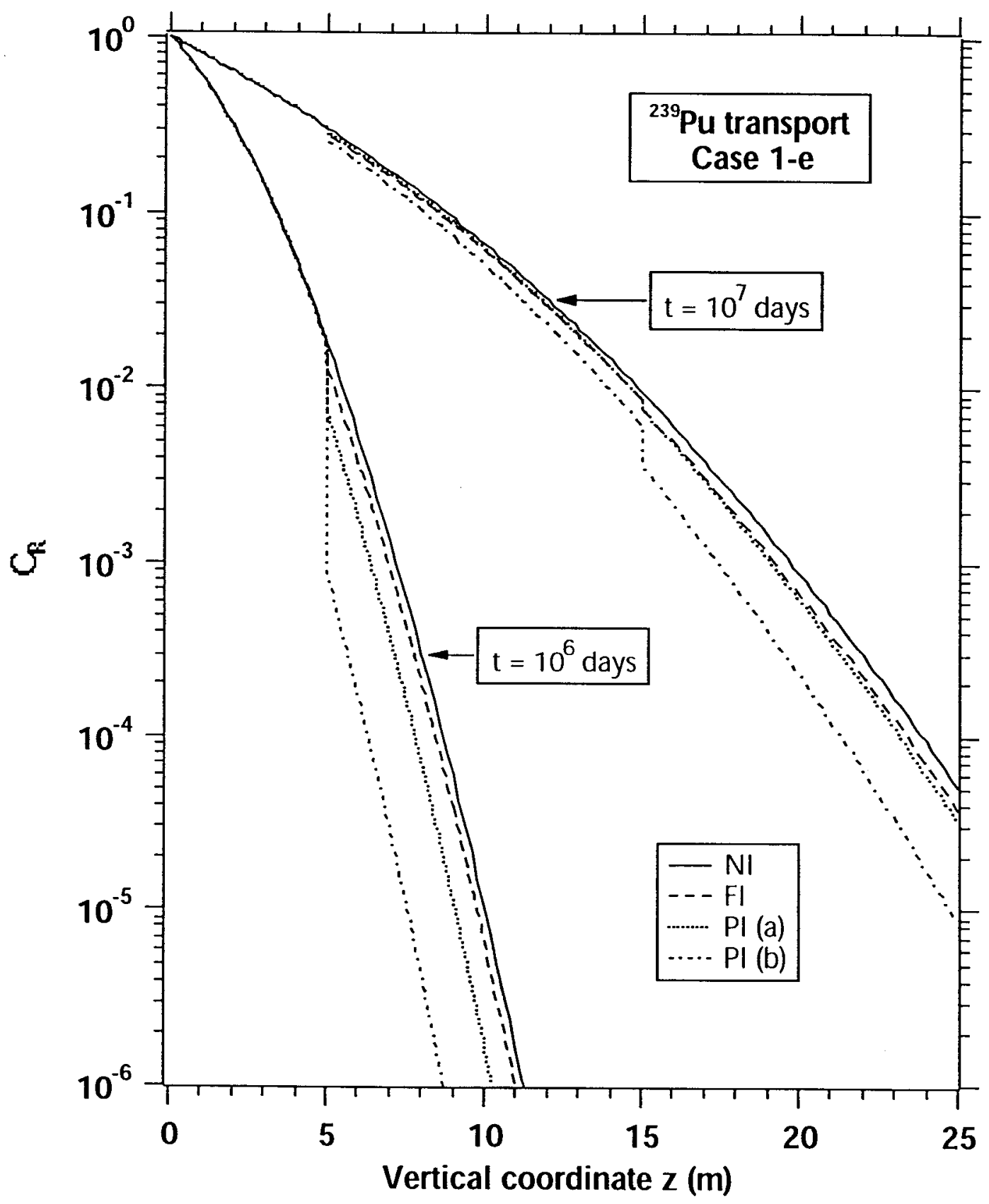

Figure 17. Effect of fracture offset (presence of interlayers) on the transport of ${ }^{239} \mathrm{Pu}$ through the layered fractured system of Case 1-e. 

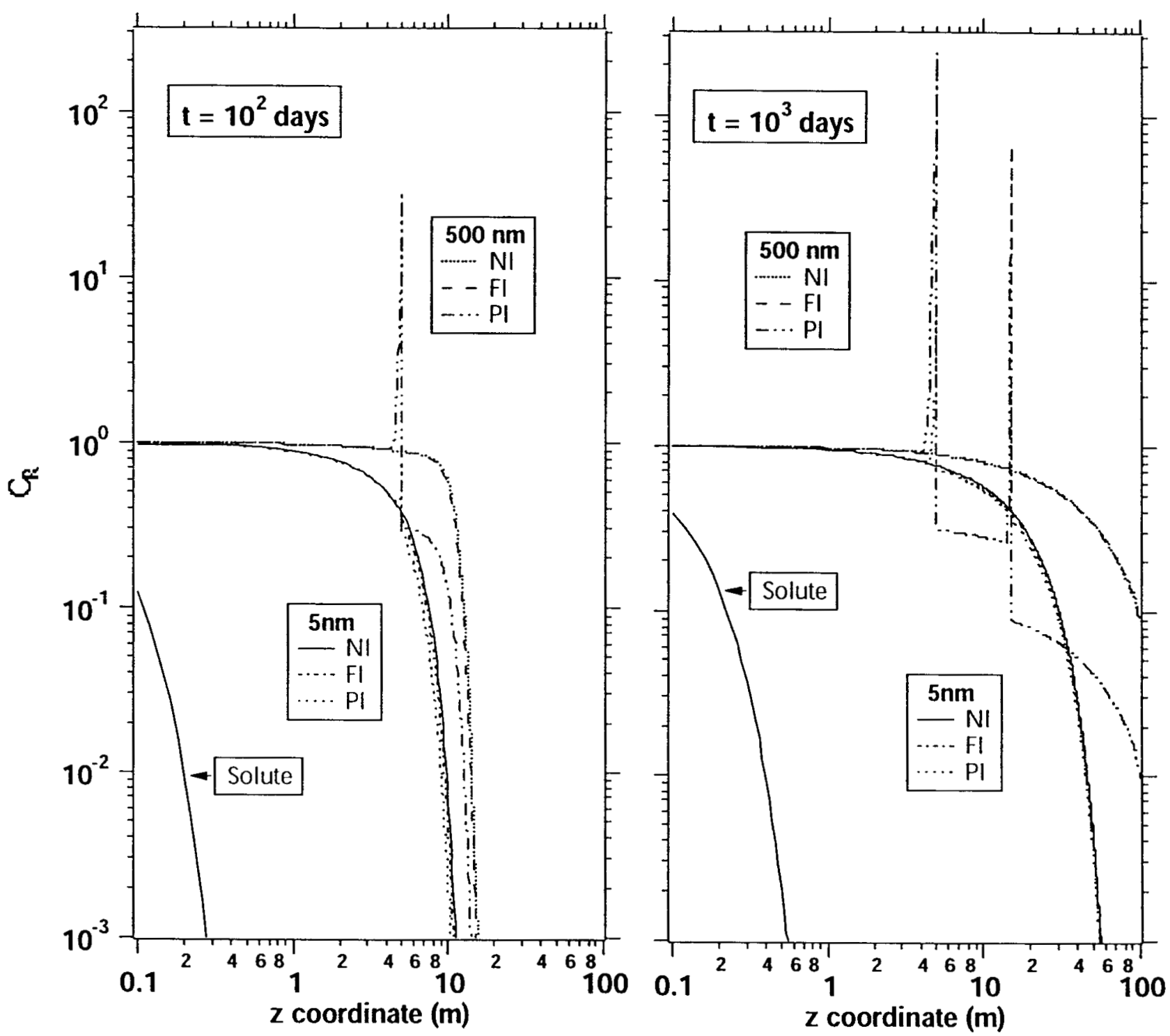

Figure 18. Fracture $C_{R}$ of the $5 \mathrm{~nm}$ and $500 \mathrm{~nm}$ radioactive colloids at $t=10^{2}$ days and $t=10^{3}$ days in the layered system of Case 2-a (no matrix filtration). 

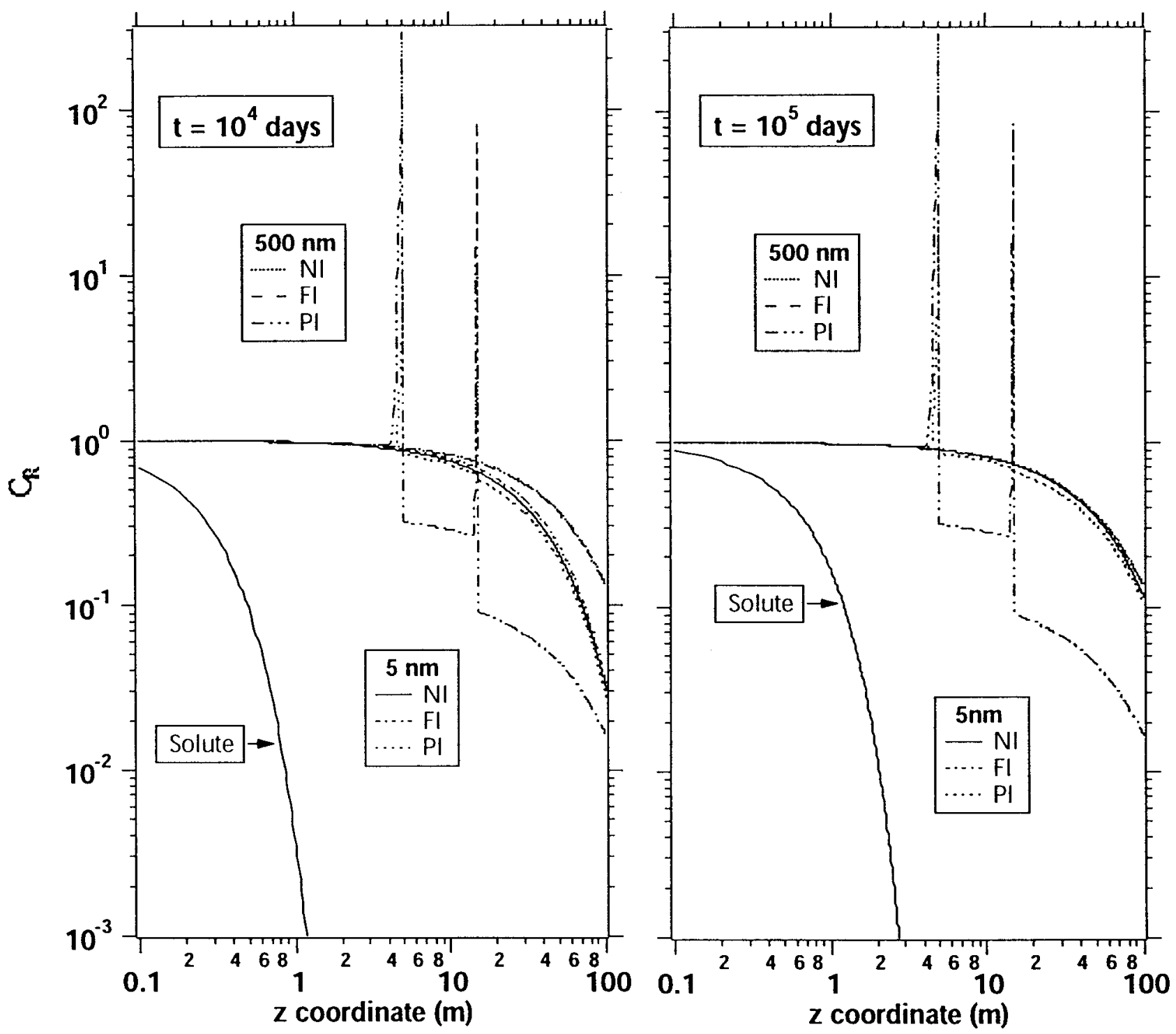

Figure 19. Fracture $C_{R}$ of the $5 \mathrm{~nm}$ and $500 \mathrm{~nm}$ radioactive colloids at $t=10^{4}$ days and $t=10^{5}$ days in the layered system of Case 2 -a (no matrix filtration). 

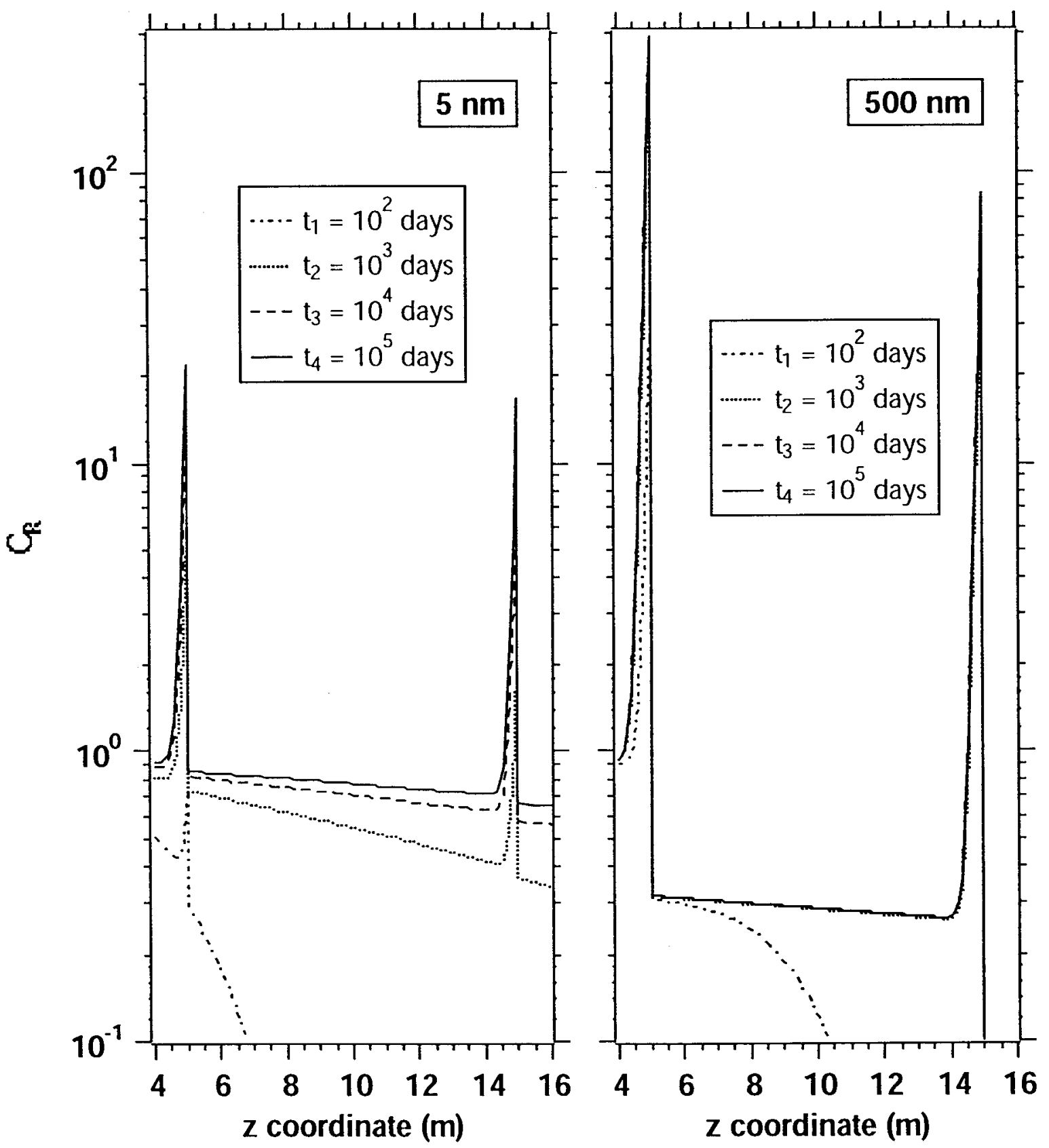

Figure 20. Detailed view of the $C_{R}$ evolution behind the sraining interfaces in Case 2-a. 


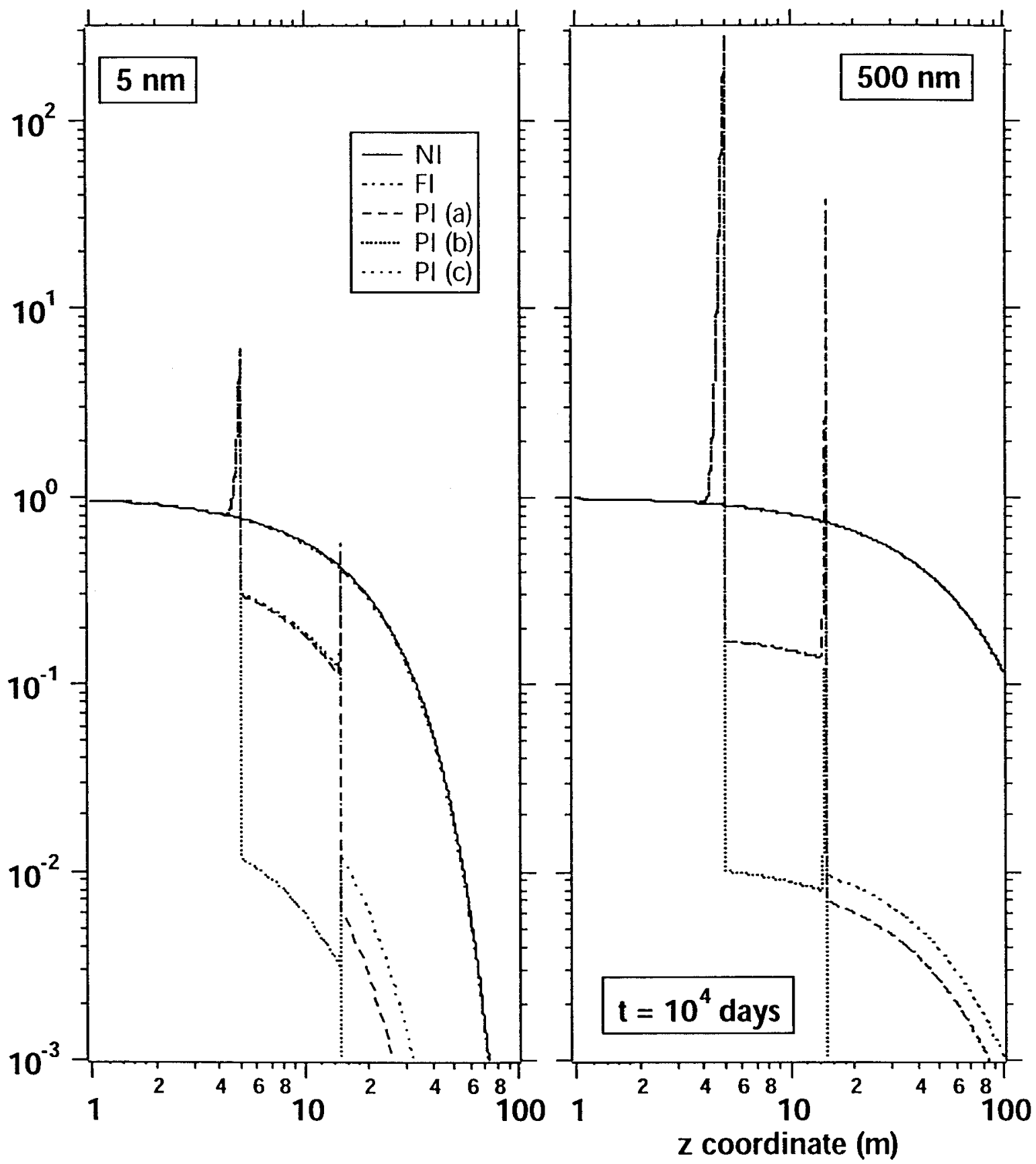

Figure 21. Fracture $C_{R}$ of the $5 \mathrm{~nm}$ and $500 \mathrm{~nm}$ radioactive colloids at $t=10^{4}$ days in the layered system of Case 2-b (with matrix filtration). 

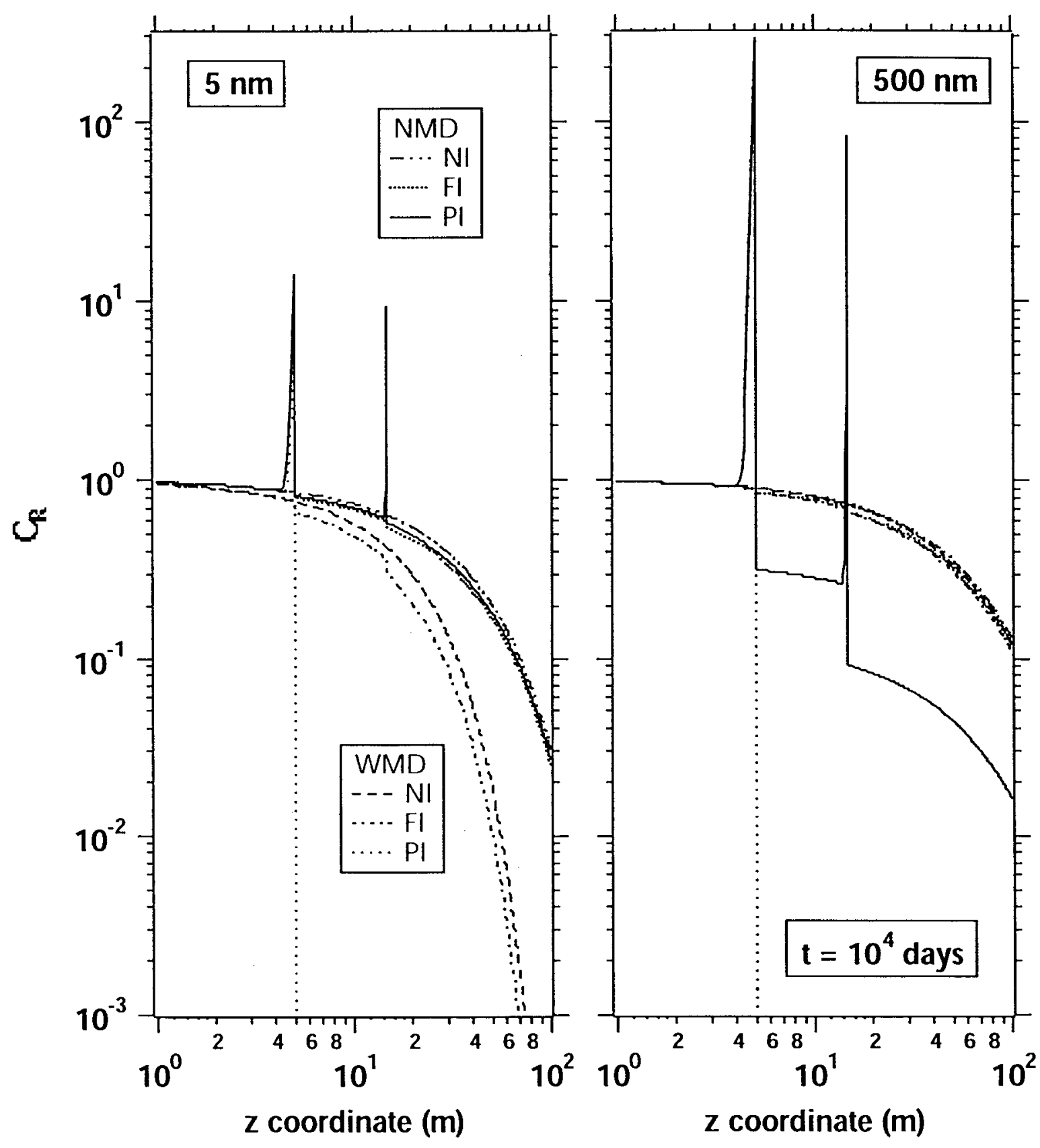

Figure 22. Fracture $C_{R}$ of the $5 \mathrm{~nm}$ and $500 \mathrm{~nm}$ radioactive colloids at $t=10^{4}$ days in the layered system of Case 2-c (WMD:with matrix deposition, NMD: no matrix deposition). 


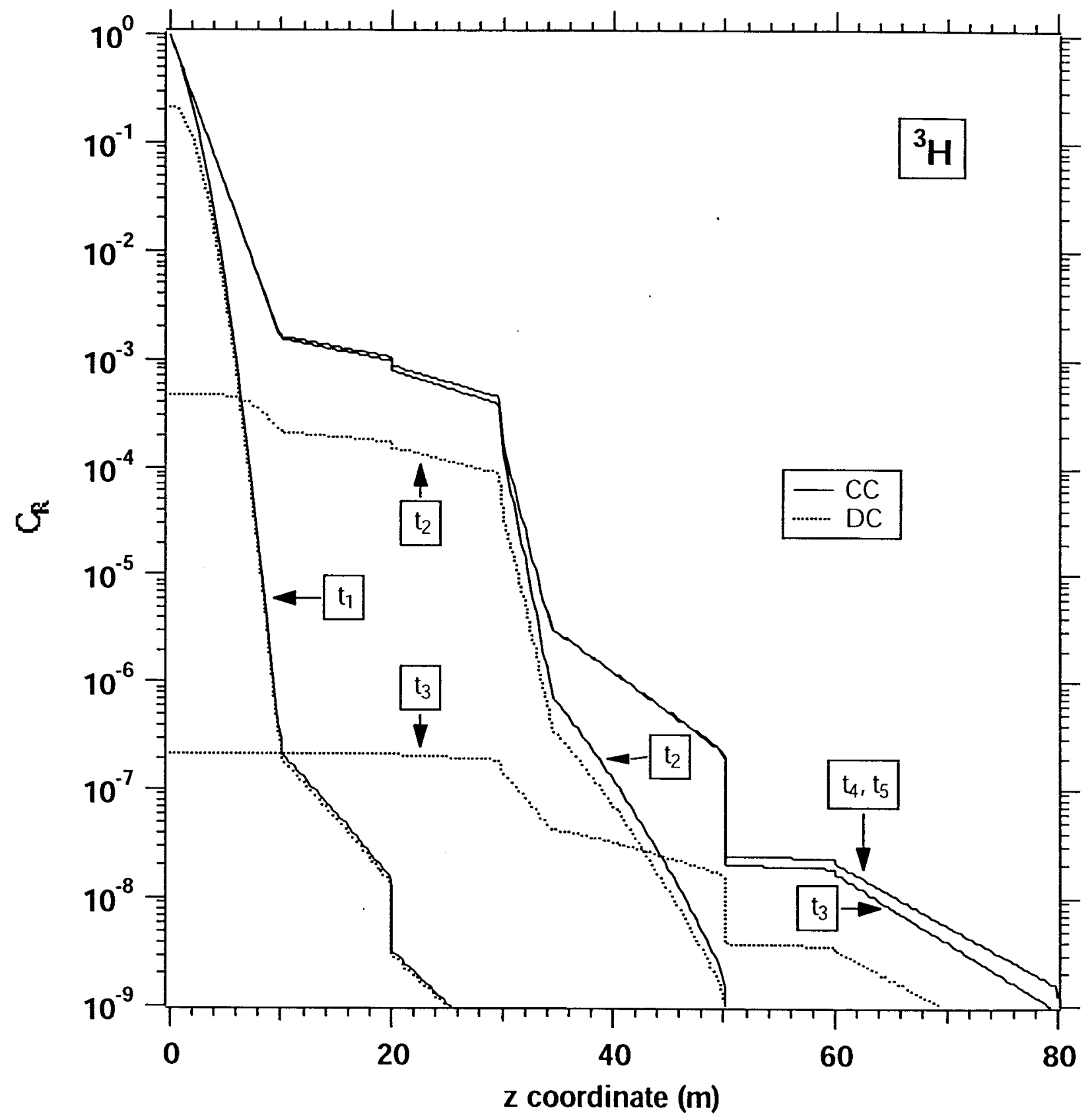

Figure 23. Fracture $C_{R}$ profiles of ${ }^{3} \mathrm{H}$ in the complex geological system of Problem 3 (CC: constant concentration boundary, DC: decaying concentration boundary). 


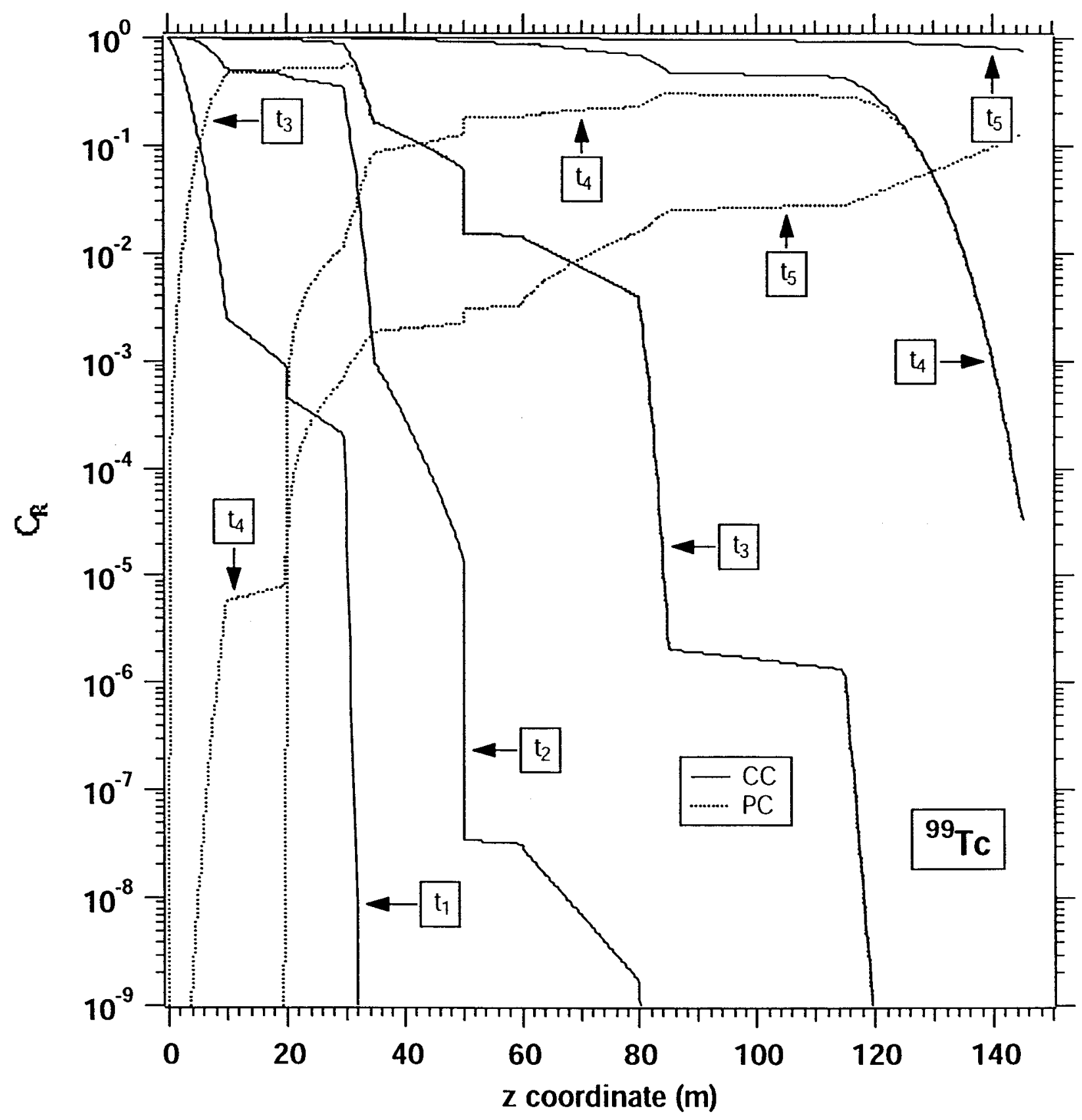

Figure 24. Fracture $C_{R}$ profiles of ${ }^{99} \mathrm{Tc}$ in the complex geological system of Problem 3 (PC: pulse concentration boundary). 


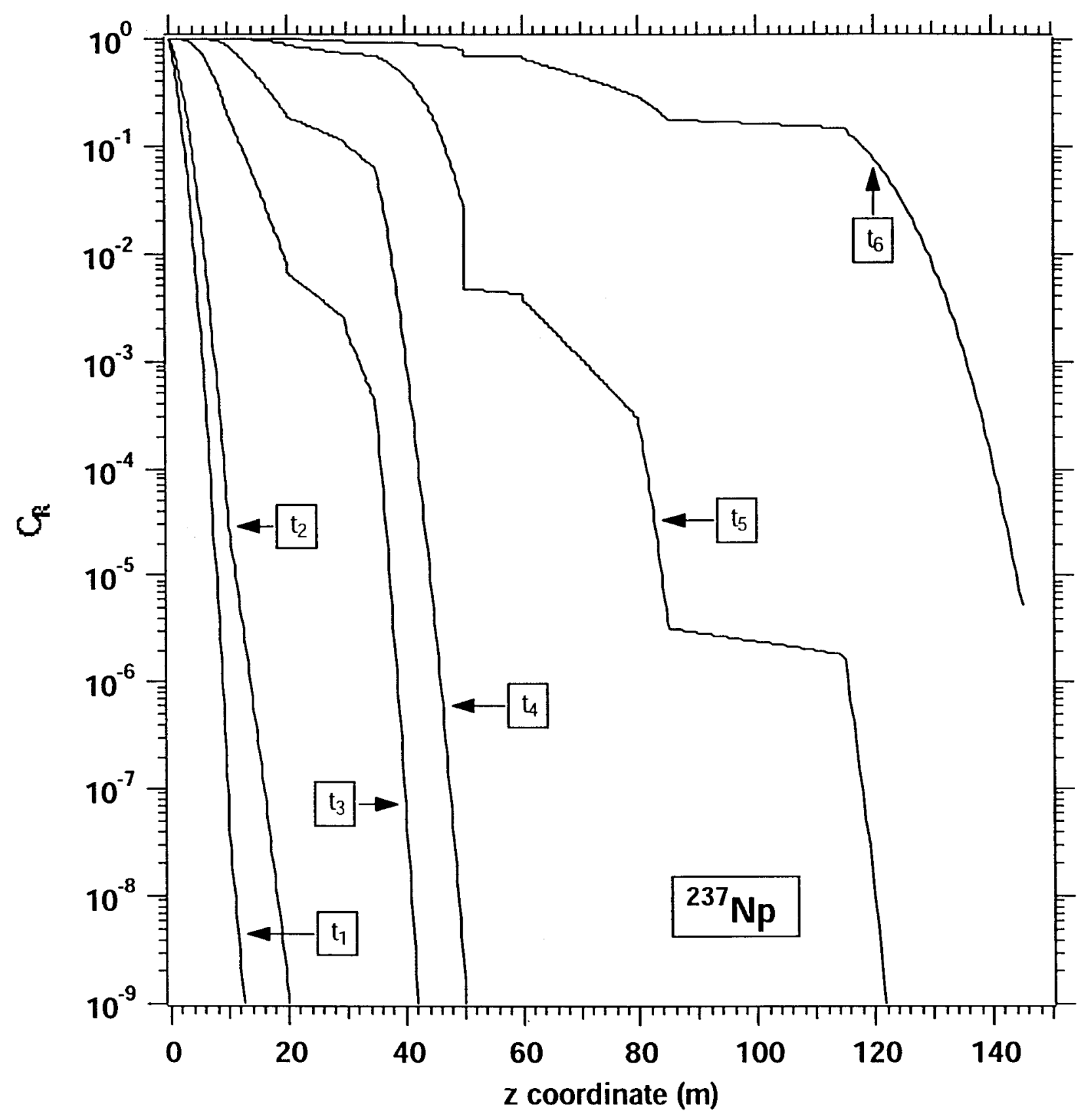

Figure 25. Fracture $C_{R}$ profiles of ${ }^{237} \mathrm{~Np}$ in the complex geological system of Problem 3 . 

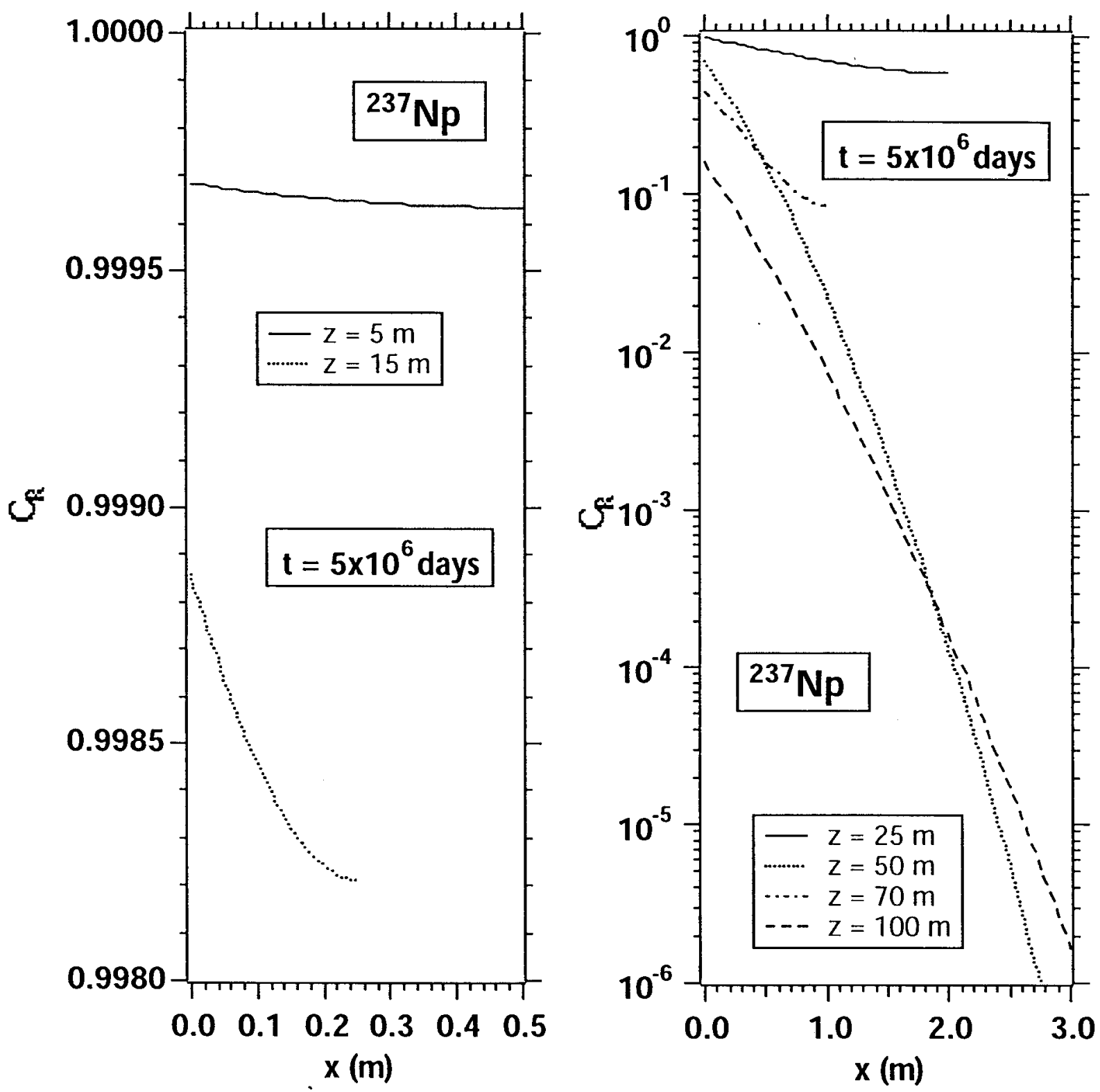

Figure 26. Matrix $C_{R}$ profiles of ${ }^{237} \mathrm{~Np}$ at different elevations in the complex geological system of Problem 3. 


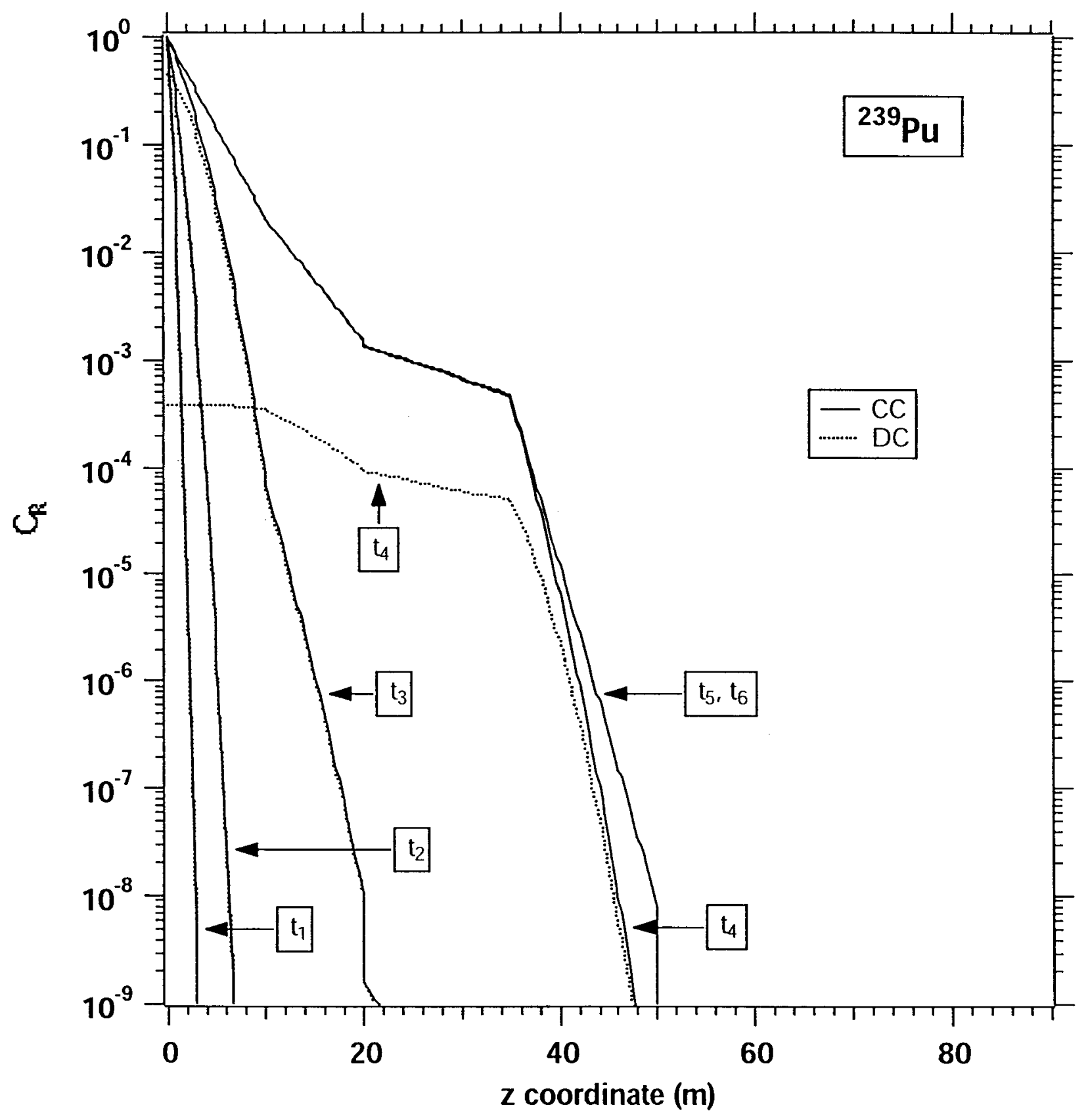

Figure 27. Fracture $C_{R}$ profiles of ${ }^{239} \mathrm{Pu}$ in the complex geological system of Problem 4 . 


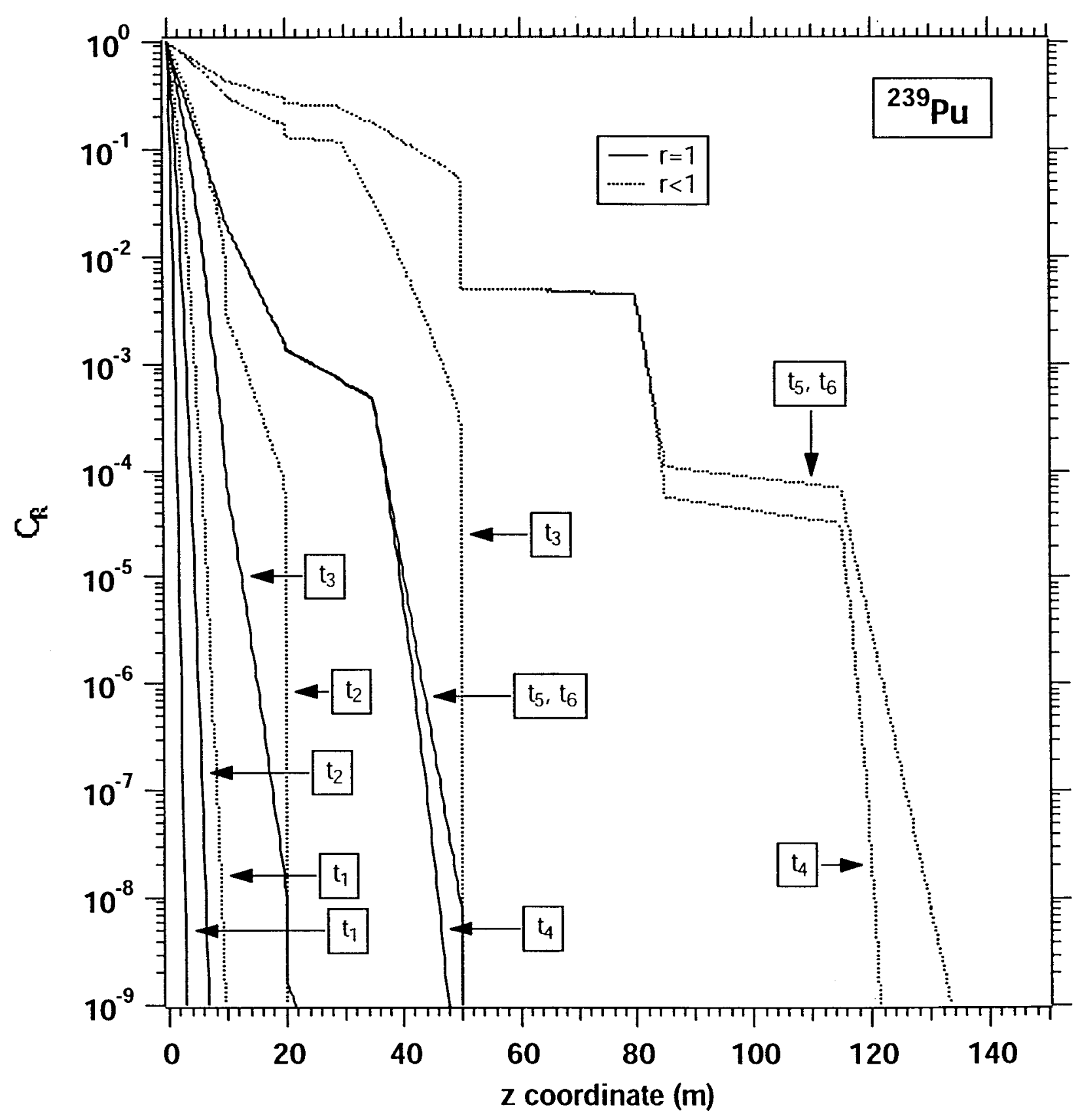

Figure 28. Effect of $r<1$ on the fracture $C_{R}$ profiles of ${ }^{239} \mathrm{Pu}$ in the complex geological system of Problem 4. 


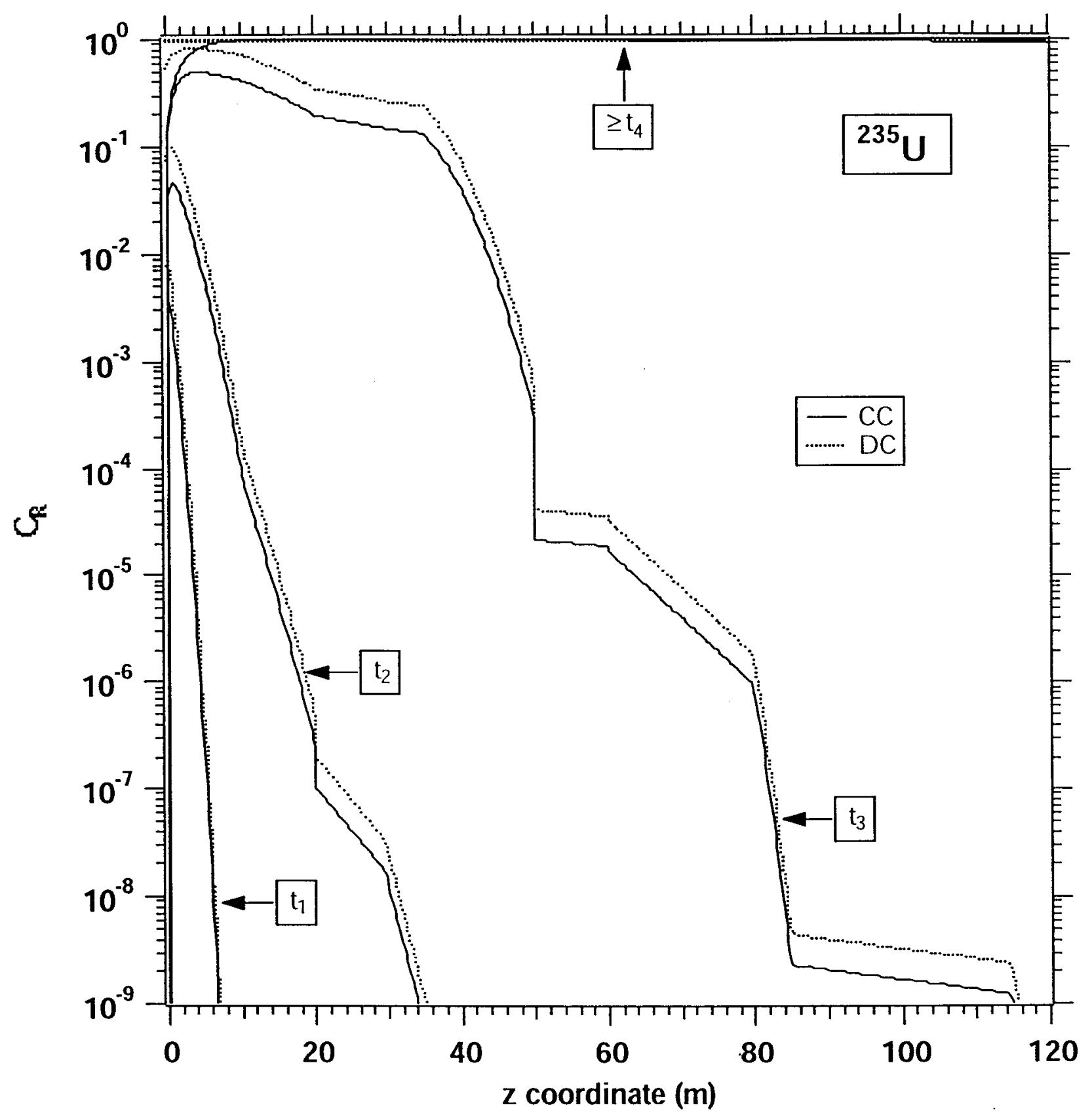

Figure 29. Fracture $C_{R}$ profiles of ${ }^{235} \mathrm{U}$ in the complex geological system of Problem 4 for $t \leq 10^{8}$ days. 


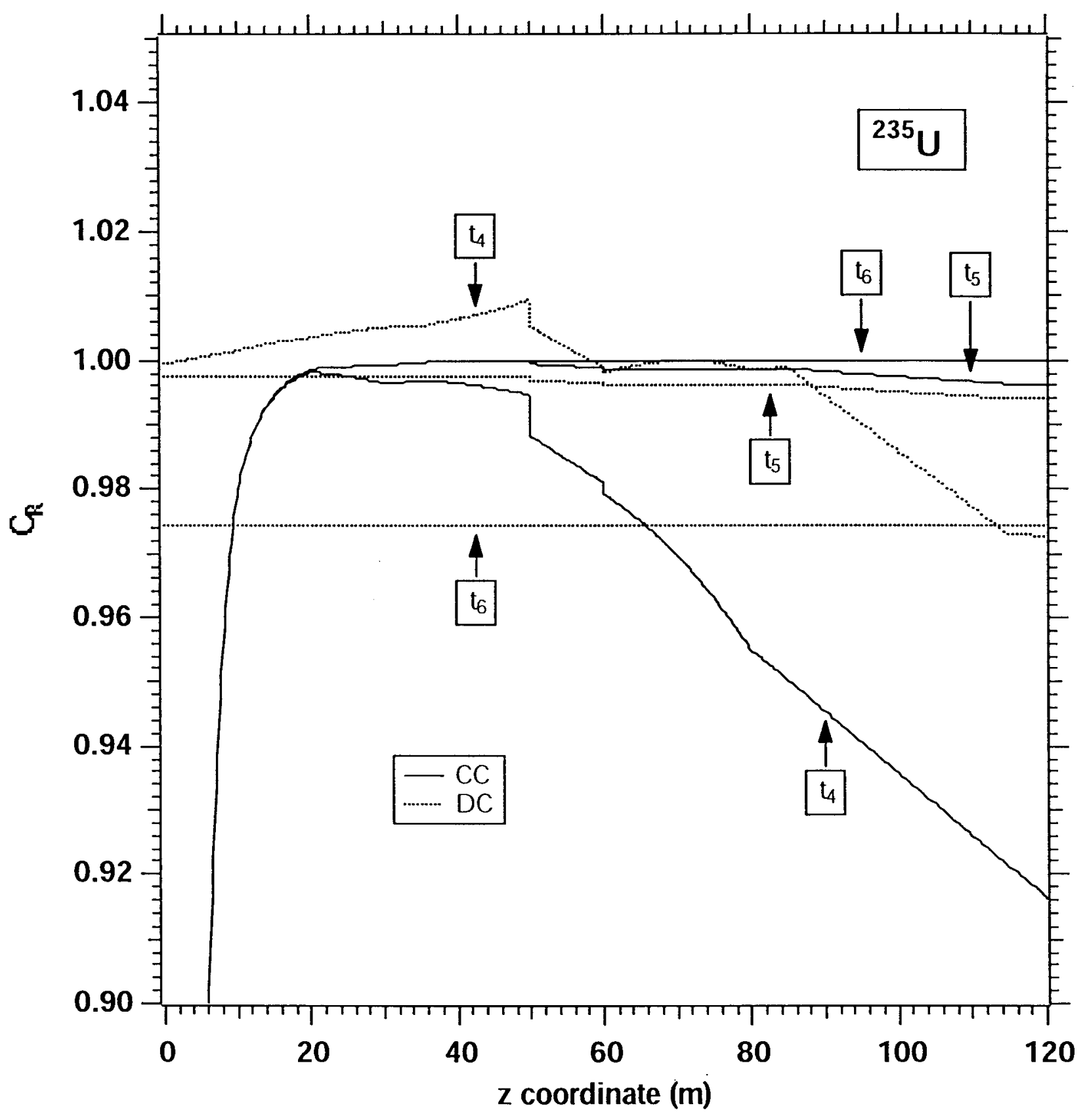

Figure 30. Fracture $C_{R}$ profiles of ${ }^{235} \mathrm{U}$ in the complex geological system of Problem 4 for $t \geq 10^{8}$ days. 


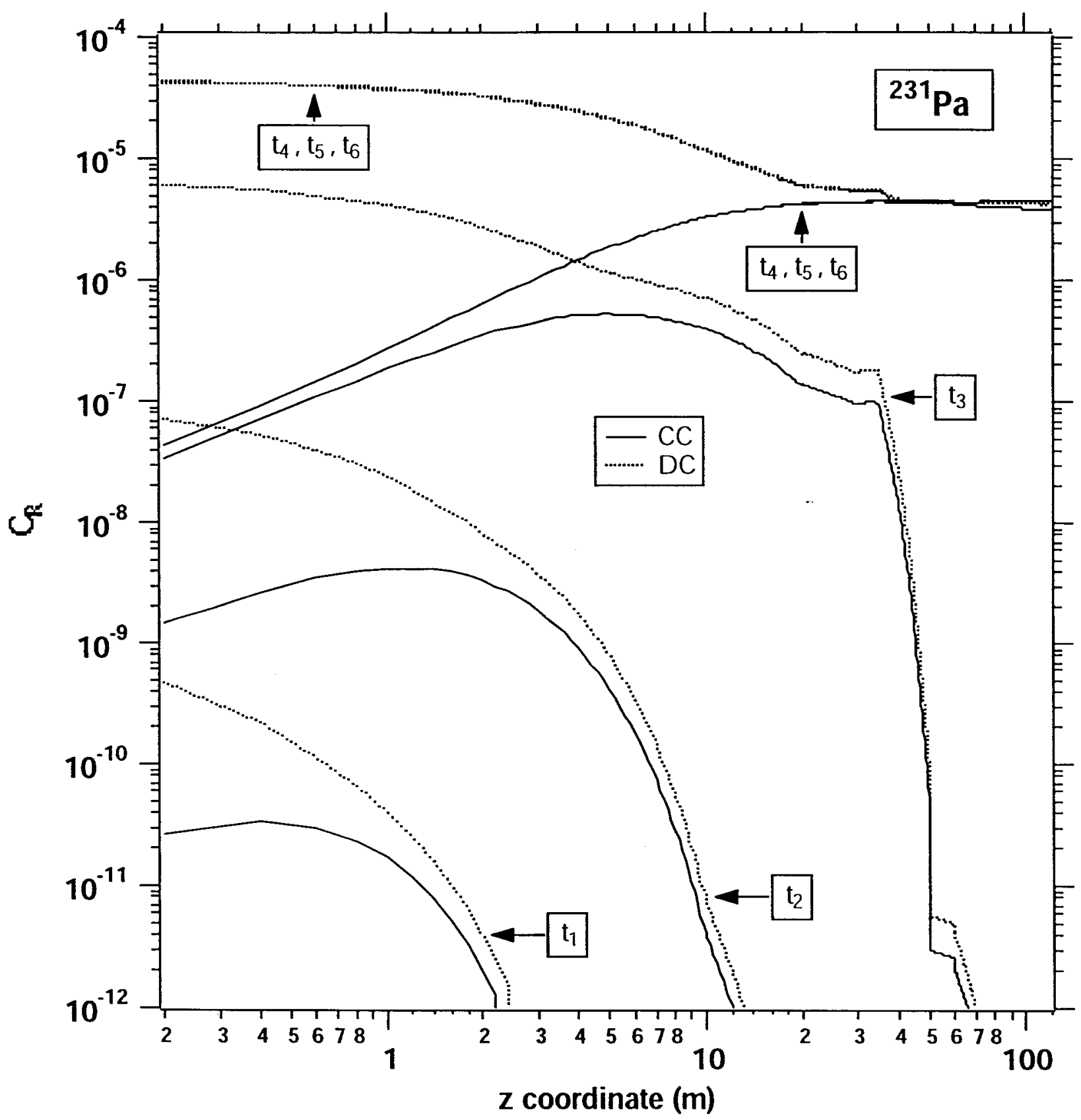

Figure 31. Fracture $C_{R}$ profiles of ${ }^{231} \mathrm{~Pa}$ in the complex geological system of Problem 4 . 


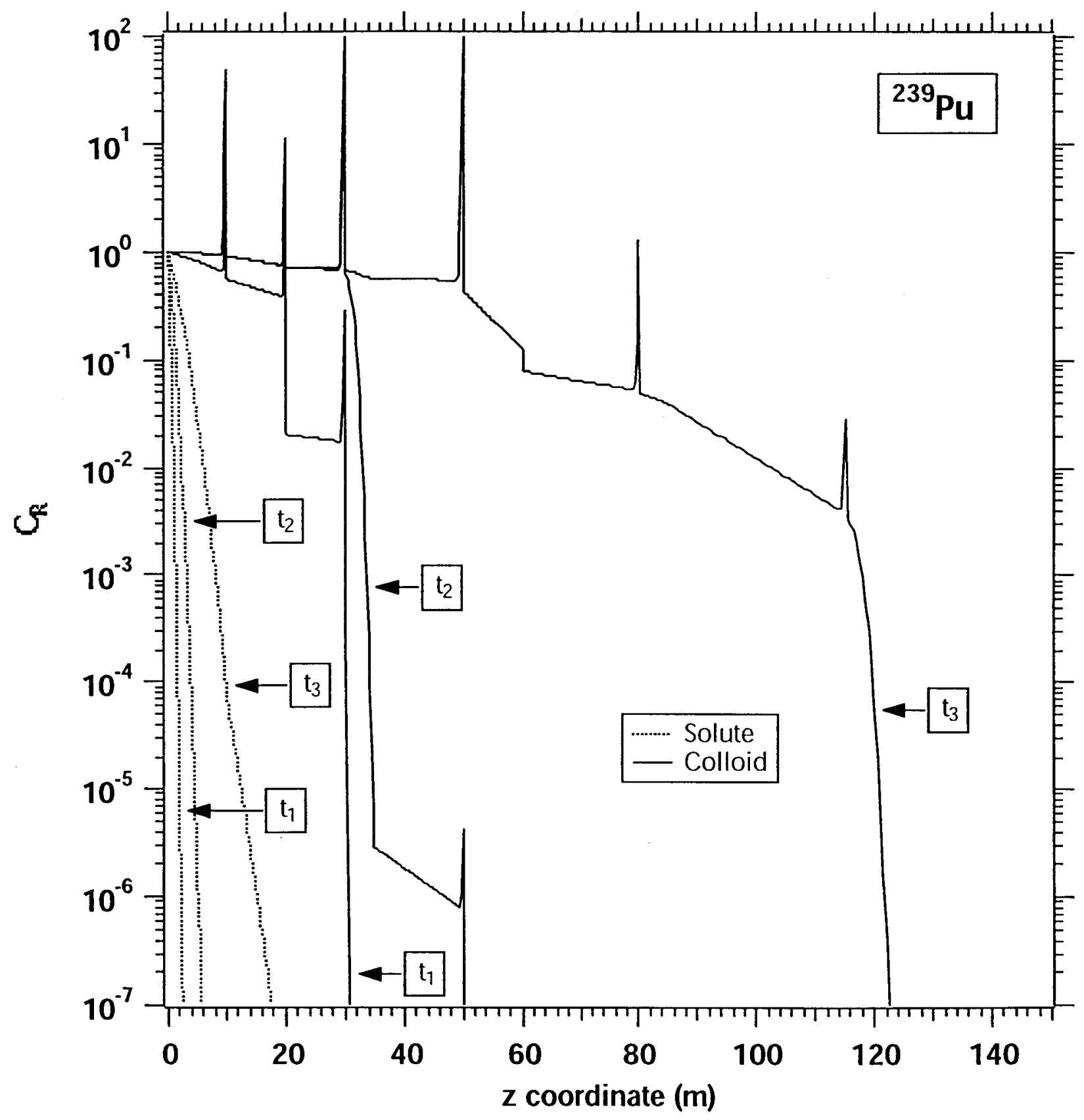

Figure 32. The fracture $C_{R}$ profiles of the ${ }^{239} \mathrm{Pu}$ colloid in the complex geological system of Problem 5. 


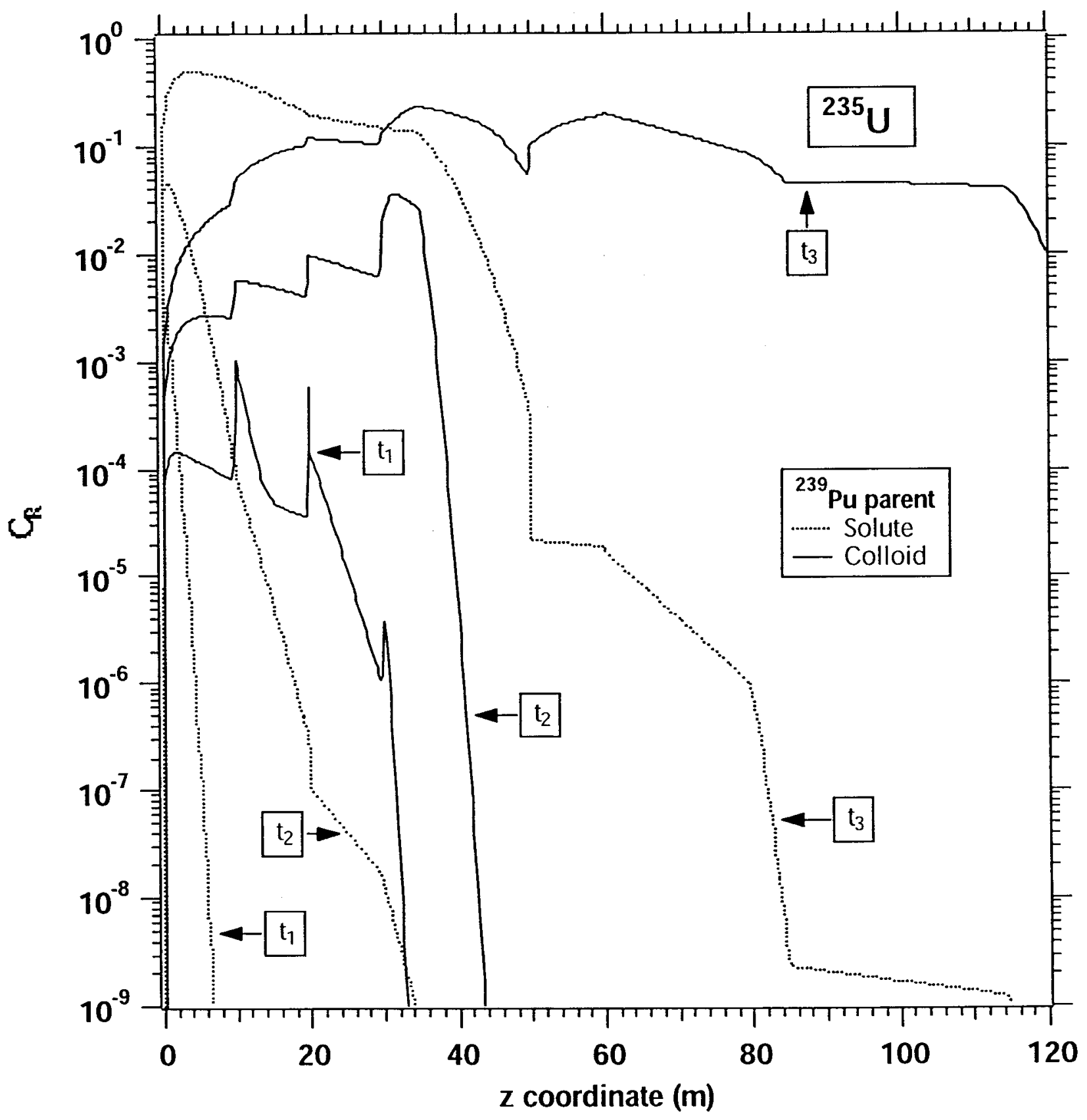

Figure 33. Fracture $C_{R}$ profiles of the solute ${ }^{235} \mathrm{U}$ daughter of the colloidal ${ }^{239} \mathrm{Pu}$ parent in the complex geological system of Problem 5. 


\section{REVIEW COORDINATION \\ PUBLICATIONS TRANSMITTAL FOR PROGRAMMATIC REVIEW}

Page 1 of 1

DATE: November 27, 2001

FROM: Bettie Moore, Review Coordination Supervisor if

BMS:mym:30174

QA: N/A

Title/Description: Semianalytical Solution ofRradioactive or Reactive Tracer in Layered Fractured Media

Product \#: N/A

W.B.S. \#: N/A

Review Purpose: LBNL Report

Due Date: December 14, 2001

Reviewers:

R.A. Levich, YMSCO, NV

D.C. Quenell, YMSCO, NV

S.P. Mellington, YMSCO, NV

Please perform a programmatic review on the enclosed document under AP-IST-004, Public Release Review, Approval, and Distribution of Technical and Non-Technical Products and return this package to the Review Coordination, Mail Stop SUM1/423, by the due date of $12 / 14 / 2001$.

If you have any questions, please contact Mayumi McDonald, Assistant Review Coordinator, at (702) 295-6660 or Danika Miller, Review Coordinator, at (702) 295-6934.

Enclosures:

1. Product for review

2. Product Review Sheet

PUBLICATION REVIEW RECEIVED BY:

Signature

Date 


\section{REVIEW COORDINATION}

\section{PUBLICATIONS TRANSMITTAL FOR PROGRAMMATIC REVIEW}

Page 1 of 1

DATE: November 27, 2001

FROM: Bettie Moore, Review Coordination Supervisor

BMS:mym:30174

QA: N/A

Title/Description: Semianalytical Solution ofRradioactive or Reactive Tracer in Layered Fractured Media

Product \#: N/A

W.B.S. \#: N/A

Review Purpose: · LBNL Report

Due Date: December 14,2001

Reviewers:

R.A. Levich, YMSCO, NV

D.C. Quenell, YMSCO, NV

S.P. Mellington, YMSCO, NV

Please perform a programmatic review on the enclosed document under AP-IST-004, Public Release Review, Approval, and Distribution of Technical and Non-Technical Products and return this package to the Review Coordination, Mail Stop SUM1/423, by the due date of $12 / 14 / 2001$.

If you have any questions, please contact Mayumi McDonald, Assistant Review Coordinator, at (702) 295-6660 or Danika Miller, Review Coordinator, at (702) 295-6934.

Enclosures:

1. Product for review

2. Product Review Sheet

PUBLICATION REVIEW RECEIVED BY:

Signature

Date 


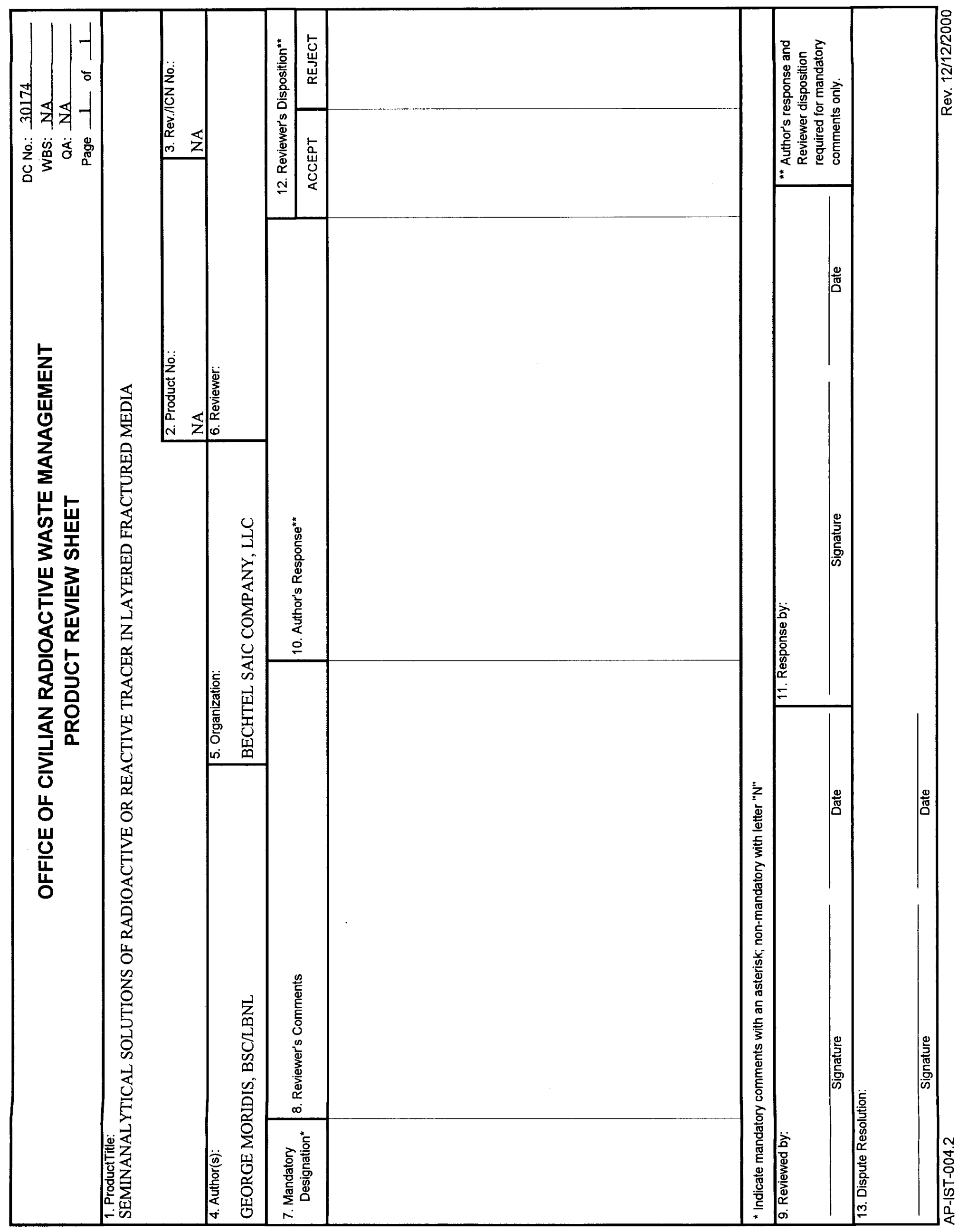


THIS TRAVELER MUST STAY WITH THE DOCUMENT THROUGH ALL APPLICABLE PROCESSES

DC TRACKING \#: 30174

DOC \# : N/A \#590

REV : $\quad$ ICN : $\quad 0$ ECN : 0 EICN : 0 DCN : $\quad 0$ PCN : 0 ADDENDUM:

DELIVERABLE :

DOC Q DESIGNATION: NA

COPYRIGHT CLEARANCE $(\mathrm{Y} / \mathrm{N}):$

ENVIRONMENTAL IMPACT STATEMENT RELATED $(Y / N)$ :

PROCESSES REQUIRED

YMP DOCUMENT QC: procedure pending

$$
\begin{aligned}
& \text { DCAR, if applicable } \\
& \text { YDAR, if applicable } \\
& 532 E, \text { if applicable } \\
& \text { RPR, if applicable }
\end{aligned}
$$

DOCUMENT PROCESSING :
YAP $5.1 Q$
QAP. 5.1
YAP $-5.8 Q$
QAP $-5-1$
PRO-IM-001

\section{DAR \\ $\mathrm{DAR} / \mathrm{DRI}$ \\ PAR}
DAR/DRI
$\mathrm{N} / \mathrm{A}$

DELIVERABLE :

YAP -30.63

PRD : $\mathrm{X}$

PRO-MG- 001

YAP -30.12

$\mathrm{N} / \mathrm{A}$

DISTRIBUTION $(\mathrm{Y} / \mathrm{N}): \mathrm{N}$ $A P-6.1 Q$

PRO-IM- 001
YDAR

SIG532E

RPR

0711, if applicable

DCAR

Verification of Content Form

Sign off Coordination sheet
DATE/INITIAL COMPLETED
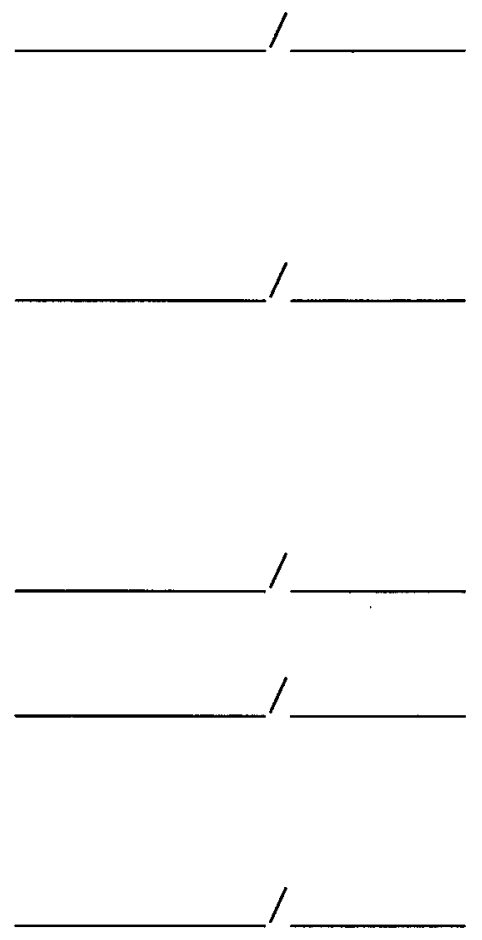

SUBMITTED BY:

POINT OF CONTACT: MORIDIS, G

ENTERED BY: OSW
PHONE : 
Subject:

Clearance Request for

Public Release of Information

To:

M. D. Voegele, M\&O
Date:

November 16, 2001

LV.RC.mym.11/2001-30174L

cc:

G. Moridis, BSC/LBNL
QA: N/A

From:

B. Moore

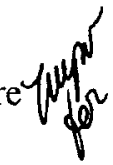

Location/Phone:

SUM1/423

(702) 295-6916

The document listed below and a Clearance Request for Public Release of Information form are enclosed.

Title: Semianalytical Solutions of Radioactive or Reactive Tracer in Layered Fractured Media

PI: $\quad$ N/A

Author(s): George Moridis

W.B.S. \#: N/A

Review Purpose: LBNL Report

Date Requested by: November 30, 2001

Product Tracking Number: $\quad 30174-2001$

When you have approved this request, please return the signed form, document, and any other records resulting from your review to Product Review (PR). PR will submit the approved document to the Yucca Mountain Site Characterization Project Office for a Programmatic Review.

If approval is contingent on modifications by the author, please notify PR. PR will notify author and coordinate modifications and, if appropriate, resubmit the document to you.

If you have any questions, please contact Danika Miller, Review Coordinator, at (702) 295-6934 or Mayumi McDonald, Review Coordinator, at (702) 295-6660.

Thank you for your assistance. 


\section{OFFICE OF CIVILIAN RADIOACTIVE WASTE MANAGEMENT \\ QA: N/A REQUEST FOR PRODUCT REVIEW (RPR) NVDC Tracking No: 30174}

\section{SECTION I.}

\section{Product Title:}

Semianalytical Solutions of Radioactive or Reactive Tracer Transport in Layered Fractured Media

\begin{tabular}{|c|c|c|}
\hline 3. Rev./ICN No: & $\begin{array}{l}\text { 4. Type of Product: } \\
\square \text { Current Technical Product } \\
\text { Product is: } \\
\square \text { Abstract } \\
\square \text { Technical Paper } \\
\square \text { Journal Article } \\
\square \text { Other }\end{array}$ & Communications Prod \\
\hline
\end{tabular}

\section{Product No:}

N/A

6. Review Purpose:

LBNL Report

\begin{tabular}{|c|c|c|}
\hline 7.WBS No.: & 8. Delive $a b$ b $/ M$ iestone No.: & 9.TD F NO :: \\
\hline N/A & N/A & N/A \\
\hline
\end{tabular}

10. Author/Product Owner: (Print Name and Phone Number) 11 . Signature: George Moridis

\section{SECTION II.}

4a. Product may have patentable material:

\section{Date Needed by Author:}

$11 / 30 / 2001$

Complete the following reviews before submitting request to Document Review:

13. TECHNICALIPEER (not required on deliverables)

$\frac{\text { John Apps }}{\text { Print Name }}$

14. AUTHOR'S MANAGER

$\frac{\text { G.S. Bodvarsson }}{\text { Print Name }}$

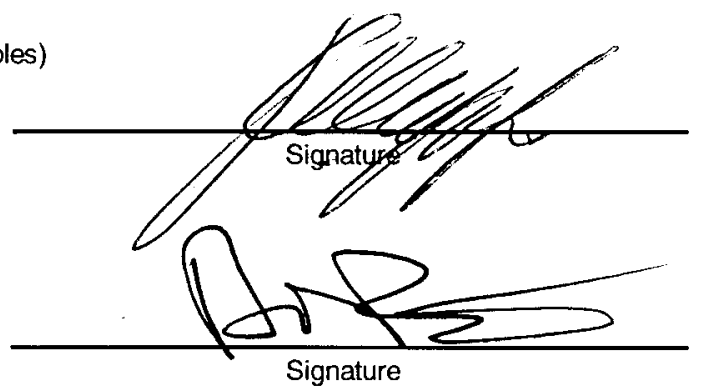

AFFECTED ORGANIZATION MANAGEMENT RELEASE FOR PRODUCT REVIEW 15. MANAGEMENT RELEASE*

Print Name

Signature

Date

* The reviewing manager may require a technical edit before releasing the document.

\section{SECTION III.}

16. Approved for distribution $\square$ Yes $\square$ No

17. Comments: 